\title{
Neural substrates of skilled vision : perceptual learning induces behaviourally relevant plasticity in human V1
}

Citation for published version (APA):

Been, M. M. (2012). Neural substrates of skilled vision : perceptual learning induces behaviourally relevant plasticity in human V1. [Doctoral Thesis, Maastricht University]. Maastricht University. https://doi.org/10.26481/dis.20121019mb

Document status and date:

Published: 01/01/2012

DOI:

10.26481/dis.20121019mb

Document Version:

Publisher's PDF, also known as Version of record

Please check the document version of this publication:

- A submitted manuscript is the version of the article upon submission and before peer-review. There can be important differences between the submitted version and the official published version of record.

People interested in the research are advised to contact the author for the final version of the publication, or visit the DOI to the publisher's website.

- The final author version and the galley proof are versions of the publication after peer review.

- The final published version features the final layout of the paper including the volume, issue and page numbers.

Link to publication

\footnotetext{
General rights rights.

- You may freely distribute the URL identifying the publication in the public portal. please follow below link for the End User Agreement:

www.umlib.nl/taverne-license

Take down policy

If you believe that this document breaches copyright please contact us at:

repository@maastrichtuniversity.nl

providing details and we will investigate your claim.
}

Copyright and moral rights for the publications made accessible in the public portal are retained by the authors and/or other copyright owners and it is a condition of accessing publications that users recognise and abide by the legal requirements associated with these

- Users may download and print one copy of any publication from the public portal for the purpose of private study or research.

- You may not further distribute the material or use it for any profit-making activity or commercial gain

If the publication is distributed under the terms of Article $25 \mathrm{fa}$ of the Dutch Copyright Act, indicated by the "Taverne" license above, 


\section{Neural substrates of skilled vision: Perceptual learning induces behaviourally relevant plasticity in human V1}

Maria Margaretha Been

September 3, 2012 
Colophon

This document was typeset by Bert Jans by using ${ }^{A} T E X$ Cover design: Marin Been

ISBN: 978-90-9027025-8

Cover design: Marin Been 


\section{Neural substrates of skilled vision: Perceptual learning induces behaviourally relevant plasticity in human $\mathrm{V} 1$}

\section{PROEFSCHRIFT}

ter verkrijging van de graad van doctor aan de Universiteit Maastricht, op gezag van de Rector Magnificus, Prof. Dr. L.L.G. Soete volgens het besluit van het College van Decanen, in het openbaar te verdedigen op vrijdag 19 oktober 2012 om 14.00 uur door Maria Margaretha Been 
Promotor: Prof. Dr. Peter De Weerd

Copromotor: Dr. Vincent van de Ven

Beoordelingscommissie:

Prof. Dr. B. Jansma (voorzitter)

Dr. M. Bonte

Prof. Dr. E. Formisano

Prof. Dr. R. Goebel

Dr. L. Jonkman

Prof. Dr. R. Vogels (Katholieke Universiteit, Leuven, België)

The work in this thesis was supported by grants from the Netherlands Organization of Scientific Research (NWO) open competitition (400-04-036), VENI (451-07-014) and VICI (453-04-002). 


\section{Contents}

1 Introduction 9

General Introduction . . . . . . . . . . . . . . . . . . . . 10

1. Evidence for learning-induced plastic changes . . . . . . . . . . . . 14

Psychophysical evidence for specificity . . . . . . . . . . . . . . 14

Neural plasticity mechanisms underlying stimulus specificity of learning . . . . . . . . . . . . . 15

Testing the locus of learning-induced plasticity . . . . . . . . . 17

2. Evidence regarding the existence of time-limited consolidation . . . 17

fMRI studies of consolidation . . . . . . . . . . . . . . . . . . . . 18

Molecular and systems-level views on consolidation . . . . . . . 18

Testing the concept of time-limited consolidation . . . . . . . . 19

3. Evidence regarding the time course of visual skill learning . . . . . 20

Psychophysical evidence and theoretical concepts on the time course of visual skill learning . . . . . . . . . . . . 20

Longitudinal investigations of neural plasticity in the time course of learning . . . . . . . . . . . . . . . . 21

Testing the time course of learning-induced plasticity and the long-term maintenance of the memory trace . . . . . . 22

4. The role of attention and feedback during visual perceptual skill

learning

Psychophysical studies of the role of attention in visual skill

learning (and related forms of experience-driven learning) 23

Insights from the neurophysiology of selective attention, relevant for visual skill learning . . . . . . . . . . . . . . . . 24

Testing the link between attention and visual skill learning . . . 25

Thesis overview . . . . . . . . . . . . . . . . . . . . . . . 25

References . . . . . . . . . . . . . . . . . 26

2 Time-Limited Consolidation and Task Interference: No Direct Link

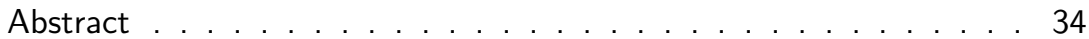

Introduction . . . . . . . . . . . . . . . . . . . . . . 34

Materials and Methods . . . . . . . . . . . . . . . 35 
Participants . . . . . . . . . . . . . . . . . 35

Stimuli, apparatus, and task . . . . . . . . 36

Training protocol . . . . . . . . . . . . . . . 36

Data and analyses . . . . . . . . . . . . . . . 37

Results . . . . . . . . . . . . . . . . . . . . . . . . 37

Behavioral interference occurs during asymptotic learning and for all time intervals . . . . . . . . . . . . . . 38

Behavioral interference is stimulus dependent . . . . . . . . 43

Discussion . . . . . . . . . . . . . . . . . . 46

References . . . . . . . . . . . . . . . . . . . . 50

3 Post-training TMS of striate cortex disrupts consolidation early in visual skill learning $\quad 55$

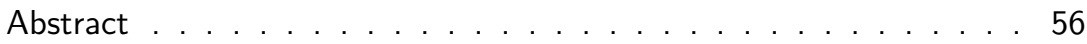

Introduction . . . . . . . . . . . . . . . 56

Materials and Methods . . . . . . . . . . . . . . . . . 57

Experimental design . . . . . . . . . . . . . . . . . . 57

Participants . . . . . . . . . . . . . . . . . . . . 59

Functional and structural MRI . . . . . . . . . . . . . 59

Preprocessing and analysis of fMRI data . . . . . . . . 60

TMS neuronavigation . . . . . . . . . . . . 60

TMS ....................... . . . . . . 61

Perceptual learning task . . . . . . . . . . . . . . 6 61

Results . . . . . . . . . . . . . . . . . . . . . . 62

Effect of offline rTMS on learning indices . . . . . . . . 62

Verification of results using JNDs and learning indices . . . . . 66

Verification of between-session consolidation (offline learning) . 66

Discussion . . . . . . . . . . . . . . . . . . 67

References . . . . . . . . . . . . . . . 71

4 Lasting memory traces of visual skill learning in human V1 75

Abstract . . . . . . . . . . . . . . . . 76

Introduction . . . . . . . . . . . . . . . 76

Materials en Methods . . . . . . . . . . . . . . . . . . 77

Participants . . . . . . . . . . . . . . . . . . . 77

Experimental Design . . . . . . . . . . . . . . . . 77

Stimuli, stimulus delivery and responses in psychophysics lab . . 78

Stimuli, stimulus delivery, and responses in scanner . . . . . . 80

Details of behavioural tasks during training in the scanner . . . 80 
Details of behavioural task in the psychophysics laboratory . . . 81

MRI data acquisition and preprocessing . . . . . . . . . . 82

Localizer stimulus and retinotopic mapping . . . . . . . . . . . 82

MRI Design for MVPA data collection . . . . . . . . . . . . 83

Analyses . . . . . . . . . . . . . . . . . 83

Results . . . . . . . . . . . . . . . . . . . . . . . . . . . . . 84

Discussion . . . . . . . . . . . . . . . . . . . . . 85

References . . . . . . . . . . . . . . . . 89

5 Neural correlates of contrast learning in the human visual cortex 93

Abstract . . . . . . . . . . . . . . . . . . . . . . . . . 94

Introduction . . . . . . . . . . . . . . . . . . . 94

Materials and Methods . . . . . . . . . . . . . . . . . . . 97

Participants . . . . . . . . . . . . . . . . . . . . 97

MRI data acquisition . . . . . . . . . . . . . . . . . . . . . 98

Stimuli and procedure . . . . . . . . . . . . . . . . . . . . . 98 98

Psychophysical design . . . . . . . . . . . . . . . . . . . 98

Stimuli, stimulus delivery in psychophysics laboratory . . 98

Task in Psychophysics laboratory . . . . . . . . . . . . . 99

Stimuli and experimental design in the fMRI scanner . . . . . . 101

Details of tasks performed in fMRI scanner . . . . . . 102

fMRI Analyses . . . . . . . . . . . . . . . . . . . . . . 103

ROI and Voxel selection . . . . . . . . . . . . . . 104

Results . . . . . . . . . . . . . . . . . . . . 105

Behavioural results . . . . . . . . . . . . . 105

FMRI data . . . . . . . . . . . . . . . . 106

Pre-Post comparison . . . . . . . . . . 106

Discussion . . . . . . . . . . . . . . . . . . . . . . 109

References . . . . . . . . . . . . . . . . . 112

6 Discussion and summary $\quad \mathbf{1 1 5}$

General Discussion . . . . . . . . . . . . . . . . . . . 116

Future perspectives . . . . . . . . . . . . . . . . . . 120

References . . . . . . . . . . . . . . . . . . . 122

7 Summary 125

8 Curriculum Vitae 129 



\section{Introduction}




\section{General Introduction}

All biological organisms continually adapt to their environment in order to survive. This implies that ongoing experiences with stimuli or events lead to changed perception and reaction to these stimuli and events when they reoccur. Helmholtz (1910) was one of the first researchers to incorporate this effect of experience into theories of perception. 'Consequently it may often be rather hard to say how much of our apperceptions (anschauungen) as derived by the sense of sight is due directly to sensation, and how much of them, on the other hand, is due to experience and training' ( $p 10)$. This statement beautifully conveys the role of experience and training in the way we perceive our environment.

Long-term changes in the response to environmental stimuli imply long-term changes in the brain. To refer to the long term permanent transformation of a system of neurons Jerzy Konorski (1948) coined the term 'plasticity'. Plasticity has become a general purpose term to refer to neural changes induced by any learning paradigm or induced by any event or stimulus. Here we define the term plasticity as a change in neuronal function that can be associated with a lasting, modified behavioural response to a stimulus.

The idea of plasticity in the developing brain is quite well established. In the first few months to years of early development of many organisms, the interaction between a fast developing brain and environmental stimuli is necessary for establishing normal brain structure and function. It is well known that infants deprived of normal environmental input will show abnormal emotional development (Joseph, 1996, 1999). Experimental evidence from monkeys (Harlow and Zimmermann, 1959) has shown abnormal emotional development after early deprivation of interactions with the mother, suggesting abnormalities in limbic structures. Likewise, manipulations of the visual environment during early development of cats (e.g., by restricting visual input to one orientation) led to severe abnormalities in the structure and function of visual cortex, and to functional blindness (Blasdel et al., 1977). Hubel and Wiesel (1963) have emphasized the importance of an interaction between environment and genetically driven development especially for the development of early sensory cortices, and proposed that the extreme malleability of brain function and structure by the environment was limited to a relatively brief 'critical period'. This term was introduced by ethologists (Spalding, 1873) and refers to strong plasticity for a brief period of time during early development.

Compared to the enormous brain plasticity in early development, plasticity in adults may appear to be small, and the extent of plasticity in early sensory areas is a matter of ongoing debate. On the one hand, there has been a long tradition of research in which topographical changes and/or neuronal tuning changes were induced in early sensory areas by massed exposure to specific stimuli (Recanzone et al., 1993). These data were complemented by comparable data showing topographical re-mapping after deafferentation (Pons et al., 
1991). On the other hand, recent studies (Smirnakis et al., 2005; Schmid et al., 2009) have suggested a complete absence of re-mapping after deafferentation, raising doubts about the validity of earlier claims of extensive plasticity in early sensory areas in adults. The data from the learning paradigms presented in this thesis are relevant for the debate whether there is plasticity in early sensory cortex (in the present thesis: V1 and low-level extrastriate cortex).

Learning paradigms implemented in the lab as well as common experience show that, obviously, also adult humans learn and form memories, implying the existence of plasticity in adulthood. Yet, until the 1960s, little was known about the structures in the brain responsible for memory formation, and memory was thought of as a monolithic ability. This conceptualization of memory provided little guidance for studies of neural brain plasticity underlying memory and learning. The dramatic outcome of temporal lobe surgery in patient $\mathrm{HM}$, which led to removed hippocampus and surrounding tissue, has shattered early unspecific ideas about memory, and taught neuroscientists many important lessons. Remarkably, HM's working memory, intelligence, and a number of specific kinds of long term memory (LTM) including various types of skill learning, seemed normal. The most pronounced deficits were present in episodic memory formation where he showed both retrograde and anterograde amnesia. Accumulating insight from HM and other patients marked the beginning of the realization that there are many different types of memory. It is now well established that memory can be divided in a sensory buffer, working memory, and long term memory (LTM). The latter has been subdivided in two main classes: explicit (declarative) and implicit (non-declarative) memory. Declarative memory includes factual knowledge about people, places and events, whereas implicit memory includes associative and non-associative learning as well as motor and perceptual skill learning (Kandel et al., 2000; Squire, 2004). The present thesis will focus on visual perceptual skill learning, a form of implicit (non-declarative) memory.

One important finding that emerged from prior memory research was the distinction between two phases of long-term memory formation; the manipulation of information for storage, which is referred to as the encoding phase, and storage itself, referred to as consolidation (Atkinson and Shiffrin, 1968; Craik and Lockhart, 1972). Consolidation has been found to exist in both explicit (Squire, 1992; Squire and Alvarez, 1995) and implicit memory (Brashers-Krug et al., 1996; Karni, 1996; Karni et al., 1998; Robertson, 2004; Robertson et al., 2004). Hence, the consolidation period is usually referred to as a period of time, extending beyond the event or training episode by several hours, in which processes take place that lead to the neural changes underlying changed perception or performance. Thus, the terms consolidation and plasticity are tightly related, but consolidation emphasizes the time course of plasticity. Importantly, two implications of the concept 'consolidation period' are currently debated. First, the idea of a 'period' implies that this process has a precise end. Second, the idea of consolidation suggests that after a certain period, the 


\section{Introduction}

resulting memory trace becomes immune against further changes. The time course of memory formation and whether it can be captured by the idea of consolidation is the second debate in the literature which is addressed by data in the present thesis, in addition to the question whether low-level visual cortex, and specifically area $\mathrm{V} 1$, contributes to it.

Visual and other skills are characterized by the fact that they typically require many training trials spaced over many days in order to result in expert performance (Gold et al., 1999; Furmanski and Engel, 2000; Sigman and Gilbert, 2000; Gold et al., 2004; Chung et al., 2006). However, as is often said, once an expert, always an expert. Gains in performance induced by sensory experience through extensive training, once acquired, largely resist decay as a function of time. This finding certainly supports the idea that training induces long term plastic changes. In the 1990's, Avi Karni and Dov Sagi published a paper on practice in texture discrimination, in which they reported that learning was retinotopically specific, orientation specific and strongly monocular (Karni and Sagi, 1991). They interpreted these results as evidence for learning to involve experience dependent changes at a level of the visual system that is retinotopically organized, where neurons show both strong orientation and ocular selectivity. The specificity of perceptual learning thus allowed these investigators to conclude that the site of plasticity induced by the training was most likely at the level of the primary visual cortex (V1). A related, influential contribution of Karni and Sagi (1993), was the finding that perceptual learning is characterized by a specific time course. They proposed that the specificity of skilled performance (and associated plasticity on low-level visual areas such as V1) only emerges during the asymptotic phase of learning, when quick improvements typical for the first few learning sessions do no longer occur, and improvements from day to day are small and require great effort. Karni and Sagi's views have been countered by alternative theories that limit the need for extensive plasticity at the lowest level of sensory systems, and in which plastic changes might occur at higher levels of the visual system, or at the level of connectivity among these areas (Ahissar and Hochstein, 2002, 2004). In short, Karni and Sagi suggested plastic changes (in tuning properties) of neurons at the lowest possible hierarchical level contributing most efficiently to the processing of the stimuli of which perceptual accuracy is being trained (Lowest level Theory). Because expertise only develops towards the end of learning (asymptotic learning phase) the low-level plastic changes would only be induced correspondingly late in learning. By contrast, Ahissar (Ahissar and Hochstein, 2002, 2004) suggested that plastic changes lead to an increase in efficiency of information readout from low-level areas by higher level areas (Reverse Hierarchy Theory, $\mathrm{RHT}$ ), without a need for extensive plastic modification of filtering properties in low level visual areas. This increased efficiency of readout would be associated with enhanced connectivity to include areas increasingly lower in the visual hierarchy, and this would underlie the emergence of expert performance during asymptotic learning. In this view, plasticity in sensory areas would 'trickle down' 
from high to lower levels as learning continues. A subset of the experiments presented in this thesis was designed to contribute to this theoretical debate between RHT and LLT on the locus and time course of plasticity.

Both RHT and LLT assume that attention is required for perceptual learning to occur. Recent studies however have shown that there are many factors that facilitate learning, including reward, feedback, and the amount of stimulus repetition, and that learning can take place (although less efficiently) in the absence of attention, or even outside consciousness (Watanabe et al., 2001; Watanabe et al., 2002; Seitz and Watanabe, 2003). Learning in the absence of attention will not be the focus of the research presented here. By contrast, we have designed our experiments to demonstrate the tight link between attention and visual skill learning, when participants are explicitly instructed that the stimuli are behaviourally relevant and to be attended.

The present introduction has identified four debates to which the thesis will contribute, and they are related to (1) the evidence for learning-induced changes in primary sensory cortex, (2) the evidence underlying the concept of consolidation, (3) the evidence regarding the across-sessions time course of learninginduced plasticity at multiple levels of sensory systems, and finally (4) the link between attention and visual skill learning. Hence, in the specific experiments within the domain of visual skill learning in this thesis, we aimed to test (1) whether there are plastic changes in the primary visual cortex resulting from skill learning; (2) whether these plastic changes can be related to a time-limited process of consolidation of the induced memory trace; (3) whether the time course of plasticity at different levels in the visual system would support RHT or LLT type theories, and (4) whether effects of attention and visual skill learning on stimuli of a range of contrasts would be comparable. The paradigm used to induce perceptual learning was a visual orientation discrimination paradigm. This is a well-established paradigm which lends itself well for an investigation of the above-specified research aims. Below, these four research aims will be framed in the context of a more in-depth literature review in four separate sections.

Prior to delving into this more specific review, it is worth pointing out that studying visual perceptual learning is interesting for many reasons besides the specific questions that motivated the present thesis. Firstly, visual skills are part of everyone's life, and thus play specific roles in many professions. Radiologists are able to see subtle changes on X-rays which for the untrained eye will remain invisible. Gynaecologists can spot a two week old embryo on an ultrasonography despite the embryo being of virtually imperceptible size (a couple of millimeters). Thus, understanding the behavioral laws of skill learning, and its underlying neuronal correlates, will provide important insights into a type of memory formation that contribute to important aspects of professional human life. Second, in a broader sense, many aspects of human cultural life (e.g., language, music, sports) and science (e.g., mathematics) rely on practice. Skill learning thereby becomes an instrument for cross-generational transmission of culture, and educational or training strategies used in that transmission can benefit from 


\section{Introduction}

insights gained from skill learning studies. Third, a deep understanding of visual skill learning can be exploited to stimulate brain plasticity to compensate for cortical damage or other deficits. In this respect, the studies in this thesis provide a set of perspectives that are complimentary to other studies from our group that emphasize neurophysiological and molecular levels of inquiry. When knowledge about behavioral properties of learning (human psychophysics) can be combined with knowledge about the locus of learning-induced plasticity (human $\mathrm{fMRI}$ ), with identification of specific subpopulations of neurons that play a crucial role (animal model neurophysiology, histology, pharmacology), and with identification of intracellular genomics/protemics changes (animal model molecular biology), biological tools may be made available to promote desirable forms of plasticity aiding learning processes in both intact and damaged brains.

\section{Evidence for learning-induced plastic changes (memory traces) at early levels of the visual system after extended training}

\section{Psychophysical evidence for specificity}

Many psychophysical learning studies have reinforced the idea that perceptual learning is often highly specific for the characteristics of the stimulus used during training. The dependence of skilled performance on stimulus parameters provides empirical constraints regarding the possible locus of learning dependent brain changes (Agranoff et al., 1965; Aberg and Herzog, 2010). In studies that have used relatively simple stimuli (lines, gratings, Gabor patches, line textures) it has been proposed that findings of high stimulus specificity can be parsimoniously explained by assuming plasticity at the lowest sensory level at which the parameter differential can be represented. Specificity and lack of transfer thus implicate an early locus of learning, because higher in the visual hierarchy, neuronal receptive fields (RFs) lack the low-level tuning properties and spatial specificity to account for specificity of low-level stimulus parameters such as position and orientation (Crist et al., 1997). Higher up in the visual hierarchy receptive field sizes are larger, cells have broader orientation tuning and generally represent more complex stimulus features. Therefore, behavioural specificity of learning is thought to reflect learning-induced plasticity in early visual areas where a more accurate representation of orientation and retinotopic location is present. Specificity of learning for the retinotopic location where participants were trained, has been found in studies of orientation discrimination (Schoups et al., 1995), for motion discrimination (Ball and Sekuler, 1987) and texture discrimination (Karni and Sagi, 1991; Ahissar and Hochstein, 1996). In addition to specificity for position, perceptual learning is also specific for the orientation of the stimulus or stimulus elements (Ramachandran and Braddick, 1973; McKee and Westheimer, 1978; Fiorentini and Berardi, 1980, 1981; Ball 
and Sekuler, 1987; Karni and Sagi, 1991; Poggio et al., 1992; Fahle and Edelman, 1993; Schoups et al., 1995; Ahissar and Hochstein, 1996; Westheimer et al., 2001). Findings of monocolarity of texture discrimination reported by Karni and Sagi (Karni and Sagi, 1991) were not replicated by a later study using a different paradigm (Schoups et al., 1995). However, taken together, there is a large number of studies demonstrating that after full training (i.e. reaching the asymptotic phase of the learning curve) improved performance shows specificity for low-level parameters of the stimulus used, which indicates plasticity in visual cortex at a very low hierarchical level.

However, not all studies find specificity for elementary stimulus parameters after learning. Some studies show that task demands can influence the degree of stimulus specificity of performance after training. These factors include task precision (Jeter et al., 2009), task difficulty (Ahissar and Hochstein, 1997), and the number of trials (Censor et al., 2009; Hussain et al., 2009). However, all of these factors are closely related with whether or not participants have reached the stage of asymptotic learning, and therefore in essence these studies do not fundamentally challenge the notion of stimulus specificity and low-level plasticity after extended (asymptotic) training. Perhaps a more challenging finding has been a report from Xiao et al. (2008) in which they demonstrated complete transfer of training from one to another location in a so-called 'double training paradigm'. Participants were trained on a feature (contrast) at location one, consecutively additional training of an irrelevant feature (orientation) took place at a second location in the visual field. This additional training caused complete transfer of feature learning (i.e. contrast) to the second location. The apparent lack of location specificity in this study could be an important finding, as it suggests that low level visual areas are not involved in the formation of skill memory (Xiao et al., 2008)., However, in this particular paradigm the training period was limited to 6 days, and although the described phenomenon is interesting, it may only confirm the high degree of generalization of skill learning in early phases of the learning curve (Ahissar and Hochstein, 1997; Karni and Bertini, 1997) Nevertheless, this study, possibly because it was published in the same time period in which other studies disputed the existence of significant plasticity in V1 (Smirnakis et al., 2005; Law and Gold, 2008), contributed to a debate about the extent and behavioral significance of plasticity in low-level sensory cortex.

\section{Neural plasticity mechanisms underlying stimulus specificity of learning}

The idea of sensory plasticity occurring at low levels of the visual hierarchy has been supported by several neurophysiological studies. In the somatosensory system, plasticity has been reported in the form of topographic remapping with representational increases after over-use of digits and representational decrease after under-use (Recanzone et al., 1992). In the auditory system both 


\section{Introduction}

topographical remapping and tuning changes in primary sensory neurons after prolonged training have been reported (Recanzone et al., 1993). In the visual system, so far there has not been evidence for remapping following training. However following orientation discrimination training, tuning properties of orientation selective neurons relevant for the trained task have been shown to undergo significant modifications in V1 (Schoups et al., 2001) and V4 (Raiguel et al., 2006). These experience induced changes in low-level sensory cortex were paralleled with analogous findings of remapping and RF changes induced by peripheral damage or deafferentation in the visual (Kaas et al., 1990; Arckens et al., 2000); somatosensory (Merzenich et al., 1983; Pons et al., 1988) and auditory (Salvi et al., 2000) domains. Taken together, these data make a strong case for experience-dependent plasticity in low-level sensory areas.

However, in a remarkable study by Smirnakis et al. (2005), high-resolution fMRI was combined with simultaneous recordings from an electrode grid implanted in monkey $\mathrm{V} 1$, to track remapping from day to day after having administered binocular damage to corresponding retinal locations. This detailed study indicated a complete lack of remapping over a period of 7.5 months. Moreover, psychophysical (Xiao et al., 2008), fMRI (Zhang and Kourtzi, 2010) and neurophysiological studies (Law and Gold, 2008) have demonstrated that perceptual learning can occur without obvious neural correlates of plasticity in primary visual cortex. In addition, some neurophysiological studies (Ghose et al., 2002) failed to find neural correlates of visual skill learning induced plasticity in V1. These studies have led to new proposals regarding the contribution of the primary sensory cortex to skilled performance. In general, these studies suggest that the memory trace for skilled performance in a visual learning task may not involve the lowest level of the visual system. As such, these studies are in agreement with the general class of 'read-out' models, of which RHT is a precursor, in which the expertise that is built up during perceptual learning is related to changes in read-out from low-level sensory cortex, and not related to modification of filtering properties through plasticity in local circuits. It is possible however, that plasticity to optimize read-out (RHT) or to optimize low-level stimulus filtering has varying relative contributions based on aspects of task and stimulus. Zhang and Kourtzi (2010) proposed that the lack of significant learning effects in V1 in their study might have been caused by their choice of stimuli; the large spacing between contour elements may have been suboptimal for the small receptive fields of $\mathrm{V} 1$ neurons.

In sum, RHT and LLT type models are not necessarily mutually exclusive. Finally, a number of experimental design limitations in previous studies of (Ghose et al., 2002) limit the interpretation of their findings (for discussion, see (De Weerd et al., 2006). Thus, although there are a number of studies contradicting the idea of significant learning or experience induced plasticity in V1, these studies have limitations as well, and they are far from completely invalidating the large number of studies that do support such plasticity. 


\section{Testing the locus of learning-induced plasticity}

To test mechanisms of plasticity underlying perceptual learning during training in visual orientation discrimination, we have used three different approaches. We have used position specificity of performance improvements after extended training as well as stimulus specificity of behavioural interference (see next section) in psychophysical experiments as a means to gather evidence supporting a role of early visual areas in the formation and maintenance of long-term memory traces for the visual skill (see Chapters 2-4). In addition, we have used $\mathrm{fMRI}$ to investigate the locus of plastic changes underlying long-term visual skill in orientation discrimination, using both univariate and multivariate approaches (Chapters 4, 5).

\section{Evidence regarding the existence of time-limited consolidation at early levels of the visual system. Psychophysical evidence for time-limited consolidation}

Time-limited consolidation implies a process of neuronal plasticity that is thought to continue for a period of time following the learning experience, and is therefore also referred to as 'latent' consolidation. Based on the idea that memory traces are vulnerable during the consolidation period, behavioural studies have used interference paradigms to test the behavioural relevance of the hypothetical consolidation processes. In tests of explicit (hippocampal) memory (Lupien et al., 1997; Smeets et al., 2008), it has been shown that stressors applied during consolidation can modulate specific aspects of memory formation. This supports the presence of active processes following the encoding phase. In tests of skill learning memory, paradigms of behavioural interference have been devised, in which an initial task (T1) is followed by an interfering task (T2), typically within minutes to hours after T1. In which impaired performance or lack of improvement on T1 the next day is seen as effective interference. If it can be shown that at a sufficiently long T1-T2 interval there is no longer interference, this would support the concept of time-limited consolidation. Several behavioural interference studies have supported this idea, both in the domain of motor skill learning (Brashers-Krug et al., 1996; Shadmehr and Holcomb, 1997) and in the domain of visual learning (Seitz et al., 2005). However, additional studies in the motor and visuo-motor domains have shown interference between T1 and T2 for very long time intervals of up to a week (Goedert and Willingham, 2002; Caithness et al., 2004). This would imply that consolidation processes last for much longer than the few-hours interval often assumed to represent the period of time-limited, latent consolidation. These observations have triggered a re-evaluation of what consolidation is, and what the molecular 


\section{Introduction}

and systems-level factors are that form the basis of consolidation processes and interference.

\section{fMRI studies of consolidation}

A number of studies have reported that sleep plays an important role in perceptual learning, by demonstrating overnight improvements in task performance (Karni et al., 1994; Stickgold et al., 2000; Mednick et al., 2002; Walker et al., 2005). Recently, several imaging studies have found sleep dependent activation in both the motor and visual domain. Walker et al (2005), trained participants on motor-skill memory and found increased activation in primary motor cortex 12 hours later, but only for participants that had slept in the intervening 12 hour interval. To asses which processes cause the improvement during sleep, Yotsumoto et al. (2009) trained participants on a texture detection task, and compared pre and post learning fMRI activity in V1 during sleep. They found enhanced activation in V1 after training, and specifically for the trained region of $\mathrm{V} 1$. These studies show activity enhancement during sleep after visual skill training, in agreement with the idea that there are consolidation processes during sleep following learning. Evidence for learning dependant activation in humans has also been found in imaging studies of resting state activity (Albert et al., 2009b; Albert et al., 2009a; Mazoyer et al., 2009; Daselaar et al., 2010). Specificity for resting state activity modulation through learning indicates that the brains default mode network activation might contribute to the consolidation of newly acquired knowledge (Albert et al., 2009a).

\section{Molecular and systems-level views on consolidation}

Predictions of behavioral interference in skill learning experiments seem often to be based on the initial molecular cascade that takes place over several hours following an experience (Brashers-Krug et al., 1996; Shadmehr and Holcomb, 1997; Seitz et al., 2005). At the cellular level, an experience triggers the expression of Immediate Early Genes (IEGs) whose protein products can act as transcription factors for Late Genes (LGs) involved in functional and structural modifications of synaptic strength between neurons (Dudai, 2004). Gene expression and protein synthesis associated with these initial cascades take place within minutes (IEGs) to hours (LGs) after neuronal stimulation, and disruption of these processes during or immediately after an experience interferes with memory consolidation (Duncan, 1949; Agranoff et al., 1965; Butefisch et al., 2000; Muellbacher et al., 2002; Luft et al., 2004). Based on the time course of these processes, it is reasonable to think of consolidation as a period lasting up to 6 hours. However, cellular plasticity related to LTM formation may comprise multiple periods during waking (Bourtchouladze et al., 1998; Korman et al., 2003; Wanisch et al., 2008) and sleep (Karni et al., 1994; Stickgold et al., 2000; Gervan and Kovacs, 2010). This may be related both to multiple molec- 
ular cascades in which LGs act as transcription factors for a subsequently activated set of IEGs, as well as to systems-related factors in which plastic changes in one network induce plastic changes in another. Systems-level considerations are an important aspect in theories of episodic memory formation proposing a slow transfer of memory traces from hippocampus to cortex (Zola-Morgan and Squire, 1990; Squire and Alvarez, 1995). After this transfer a memory trace is believed to be fully consolidated, though this proposal is debated (Nadel and Moscovitch, 1997; Nadel et al., 2000). Systems level aspects of consolidation have been considered less explicitly in the domain of skill learning, and hypotheses about the length of consolidation have been typically guided by the time course of the initial cascade of cellular events following a learning experience (Brashers-Krug et al., 1996; Shadmehr and Holcomb, 1997; Seitz et al., 2005). As shown by other studies that suggest memory traces remain vulnerable over very long periods that are incompatible with the idea of time-limited consolidation (Goedert and Willingham, 2002; Caithness et al., 2004). There is another important systems level consideration related to consolidation. For both implicit and explicit memories, efficient storage requires a representation of different memory traces in sufficiently segregated neural networks. If consolidation is to be a useful concept, it must include a component of segregation. Thus, the concept of consolidation has a molecular component operating on a time scale of hours to days as well as several systems-level components that may operate over longer time scales. Moreover, the systems-level and molecular levels are intimately connected. From these considerations, the simple concept of a single time-limited period of consolidation seems untenable a priori, and it is possible therefore that concepts of consolidation as tested in a number of behavioral interference studies were too simplistic.

\section{Testing the concept of time-limited consolidation}

To test the existence of consolidation, we used two different approaches. In one approach (Chapter 2), we have used behavioural interference, in which training on an initial orientation discrimination task was followed by subsequent training in orientation discrimination at reference orientations different from the one used in the preceding task. In this experiment, we tested interference and hence the existence of time-limited consolidation processes after each training session over a period of 3 weeks (15 daily training sessions). In the other approach, we used transcranial magnetic stimulation (TMS) administered within an hour after a skill learning experience to interfere with consolidation (Chapter 3 ). The data from these two methods to induce interference were used to evaluate the time course of consolidation and the contribution of low-level areas (V1) to consolidation early on in the learning curve. 


\section{Evidence regarding the time course of visual skill learning and associated plasticity at different levels of the visual system.}

\section{Psychophysical evidence and theoretical concepts on the time course of visual skill learning}

The time course of perceptual learning reveals different stages, and a learning curve can be subdivided in three stages. The first session can be considered the first stage. In the first session, there is usually strong within-session learning. Sometimes, these fast within session performance improvements occur literally within seconds or minutes, after a very limited number of trials when high quality input is provided (Schoups et al., 1995; Ahissar and Hochstein, 1997; Sakai et al., 2003). The fast improvement indicates a fast form of plasticity in high-level centres outside the sensory system. Whether the first session already is followed already by a consolidation period in which plastic changes occur in specific areas of the visual system is unknown, and this is one of the research questions addressed in this thesis. A second phase of the learning curve is characterized by fast session-to-session improvements in performance, which however are not realized within the individual sessions, but rather in a latent period in between sessions, referred to also as the consolidation period (see section 2). In a final asymptotic phase of the learning, the session-to-session performance gains become increasingly smaller (Karni and Sagi, 1993; Karni, 1996; Karni and Bertini, 1997).

During the first, and possibly also in part the second phase, learning is hypothesised to reflect the construction of strategies that are required to solve the perceptual task, and the acquired performance gains are not stimulus-specific. It is thought to involve top down processes that are crucial for the detection of relevant stimulus features (attention, see section 4). After initially relatively fast learning during the second stage, slower learning occurs in the third stage of perceptual learning, during which gains in performance become specific for the stimulus. These slower forms of learning are thought to involve structural modifications in low-level visual cortex. They may involve, both the strengthening of connectivity with increasingly lower-order areas of the visual system (Ahissar and Hochstein, 2002, 2004; Ahissar et al., 2009), and the modification of local circuits at the very lowest levels of cortex for the automatic filtering of relevant aspects of the stimuli (Karni and Sagi, 1991, 1993; Schoups et al., 2001). This learning stage involves the uncoupling of the performance increments from top-down processes that initially guide the learning and the selection of the relevant stimulus parameters (and neuronal population) for the learning. After asymptotic performance has been reached, and in the absence of interfering factors (see Section 2), there seems to be little forgetting. Skill-related 
memories are normally retained over several weeks, up to several years (Karni and Sagi, 1993).

\section{Longitudinal investigations of neural plasticity in the time course of learning}

Although studying the time course of plasticity in visual areas relevant for visual task performance and skill learning is essential to gain a better understanding of the neuronal plastic mechanisms underlying skill learning, there are only a couple of fMRI studies that have attempted to do so. One of these fMRI studies was conducted in the auditory domain. Reed et al. (2011) observed topographical remapping in A1 in the course of learning an auditory task, but once the skill was fully learned, the topographic map reverted to its default. This indicates that A1 was only involved in the acquisition of the perceptual skill, but not in the long-term maintenance of the memory trace (Reed et al., 2011). This is an interesting finding, but it goes against a significant body of evidence in the visual system from both neurophysiological (Schoups et al., 2001) and fMRI studies (Furmanski and Engel, 2000; Yotsumoto et al., 2009) that do suggest lasting effects of neural plasticity in V1 after prolonged skill learning. Thus, the idea that correlates of plasticity in primary sensory cortex would only be relevant for acquisition, but not for memory formation may not hold in the visual system, and may point to differences between the two sensory modalities. Nevertheless, in a longitudinal study of visual skill learning from Yotsumoto et al. (2008), similar findings were reported. They found enhanced neural activity to the stimuli at the trained location in the visual field (compared to an untrained control location) especially midway along the time course of learning, and a disappearance of this activity enhancement at the end of the learning curve, although the behavioural performance level remained elevated. Interestingly, while most studies test the effect of skill learning by measuring (fMRI) activity in response to stimuli that are not attended (e.g., by imposing a demanding fixation task), the data in Yotsumoto et al. (2008) were collected while participants performed the task and hence attended to the stimuli. When participants reach asymptotic performance, it can be expected that the role of attention becomes less, and therefore the reduced fMRI activity likely represented the withdrawal of attention as participants became more skilled at the visual task in which they were trained. Altogether, the evidence for the role of $\mathrm{V} 1$, and visual cortex in general in both the formation of new memory traces in the course of learning, and the long-term maintenance of these memory traces is scant, and this is due to the lack of studies that track activity changes in low-level cortex as learning progresses.

The lack of a longitudinal approach to (visual) skill learning is surprising, also because such approach is essential to distinguish RHT and LLT class theories. RHT and read-out theories in general predict that plasticity starts early in training, in high-level areas of the sensory system. RHT proposes that visual skill 


\section{Introduction}

acquisition and performance enhancement reflect increasing access to increasingly lower levels of the visual system, to read out increasingly detailed aspects of visual stimulus information. The synapses involved in the feedforward-feedback loops that would be involved in read-out thus would undergo plasticity, and as this loop would be expanded to included areas at increasingly lower levels, traces of plastic changes can be expected to occur at increasingly lower levels of the system as a function of learning. According to LLT, the strongest plasticity should be observed at the lowest level of the visual system involved in representing the trained stimulus parameter, and plastic changes are expected only towards completion of learning. fMRI provides a wonderful opportunity to track activity changes related to plasticity in multiple visual areas at the same time, and therefore offers the possibility to test the two categories of theories that are used to explain visual skill learning. In addition to fMRI, there are currently neurophysiological approaches that are well suited to measure the time course of skill learning induced plasticity. Whereas classical methods involve the insertion of single unit electrodes or depth probes in one or more visual areas on a daily basis, there are currently methods that permit the long-term implantation of tetrodes (e.g., F. Wilson, Wilson \& McNaughton), electrode grids (Smirnakis et al., 2005) and subdural Ecog grids (Rubehn et al., 2009), which permit the recording of the same populations of neurons in multiple brain regions over weeks and months, during the course of skill learning. Surprisingly, to date these methods have not been applied in the context of longitudinal studies of skill learning.

Another issue that has received little attention is whether memory traces formed towards the end of training are maintained in the weeks and months following the end of training. This is surprising, as it is an essential aspect of the idea that there is plasticity in primary sensory cortical areas (here, V1) that is relevant for the maintenance of expert behavior. To our knowledge, no fMRI studies have addressed this issue to date, although some neurophysiological studies in monkeys did record data in V1 (Schoups et al., 2001) or V4 (Raiguel et al., 2006) over a prolonged period following the end of training, suggesting that memory traces induced by visual skill learning do indeed last for months. Altogether, it is clear that the time course of plasticity at different levels of the visual system during visual skill learning, and the long-term survival of the memory trace are scarcely studied questions in the field.

\section{Testing the time course of learning-induced plasticity and the long-term maintenance of the memory trace}

The issue of long-term maintenance of learning-induced memory traces in V1 will be addressed by using Multi Voxel Pattern Analysis (MVPA) in Chapter 4. This technique will be used to test whether almost two months after concluding an extended training period distributed activation patterns in 'trained' regions of V1 still contain more information to classify grating orientation than response 
patterns in 'untrained' regions. In Chapter 5, a number of approaches have been applied to investigate the presence of time courses of plasticity that could be informative to decide between read-out theories or theories that propose plasticity is limited to the lowest levels of the visual system.

\section{The role of attention and feedback during visual perceptual skill learning}

\section{Psychophysical studies of the role of attention in visual skill learning (and related forms of experience-driven learning)}

A dominant view is that for efficient perceptual learning to occur, the trained stimuli must be attended (Shiu and Pashler, 1992; Ahissar and Hochstein, 1993; Karni and Bertini, 1997; Ahissar et al., 1998; Schoups et al., 2001). This view is common to both LLT and RHT theories of perceptual learning. The underlying rationale for this view is that it would be undesirable for the visual system to permit stimulation from the environment to induce plasticity too easily. In light of the plethora of information in our environment, this would lead to a highly unstable system. However, adaptive changes in response to relevant stimuli (selected by attention) are desirable, and they can be achieved by favouring plasticity for relevant stimuli or stimulus features.

In agreement with the view that attention gates learning, several studies have confirmed that visual perceptual learning only occurs for those features that are attended (task relevant features). For example, in tasks where two features were presented and only one of the features was attended, perceptual learning only occurred for the attended feature (Shiu and Pashler, 1992; Ahissar et al., 2001; Schoups et al., 2001)

Note that attention is often closely related to feedback. An interesting piece of evidence supporting the necessity of conscious effort, or at least its facilitating influence, is the demonstration that trial by trial feedback of correctness facilitates visual perceptual learning (Fahle and Edelman, 1993; Herzog and Fahle, 1997). However, not all studies in which feedback was manipulated ( $\mathrm{Lu}$ et al., 2011) (for review) are in agreement with the idea that feedback is required for learning. Indeed, visual perceptual learning has been found to also occur in the absence of feedback. However, even without feedback, there can be effort or attention directed towards the target stimulus (Poggio et al., 1992; Shiu and Pashler, 1992; Weiss et al., 1993), and therefore the issue whether feedback or attention influence learning and plasticity should be considered as separate questions.

In addition to learning without feedback, there have been studies showing visual skill learning without attention, and even without conscious awareness of the stimuli. In these studies, subthreshold stimuli were presented outside focused attention during an initial exposure period, while an irrelevant task at 


\section{Introduction}

fixation was performed (Watanabe et al., 2001; Watanabe et al., 2002; Seitz and Watanabe, 2003). Nevertheless, the never-perceived stimuli showed an advantage in subsequent detection/discrimination learning compared to other stimuli that had not been pre-exposed. This may have been due to the association with feedback in the central fixation task. This suggests that training with both attended and non-attended (non-conscious) stimuli can be facilitated by feedback. Similarly, task irrelevant learning can be boosted by attending to the feature (Gutnisky et al., 2009), and rewards can gate plasticity and learning for both task relevant and irrelevant (non-attended) features (Franko et al., 2010).

In addition to the effects of attention/consciousness and reward/feedback, mere stimulus presentation can play a factor in learning. For example, Dinse and colleagues have shown that active performance of a tactile task can improve through passive, suprathreshold but unattended stimulation (Godde et al., 2000; Dinse et al., 2006). The evidence for this form of passive learning is not restricted to the motor cortex. In the visual cortex, there have been interesting observations supporting reorganisation in low-level sensory cortex after passive stimulation without reward, feedback, or attention. In mice v1, plasticity was found after prolonged passive stimulus exposure (Frenkel et al., 2006). Hence, there are multiple factors that can contribute to (visual) skill learning, including numbers of trials (exposure), feedback, reward, and attention. However, learning is by far the most efficient when it is guided by attention. The learning paradigms we have used in the present thesis always involved selective attention to a relevant parameter of the visual stimulus, and fMRI correlates of learning (Chapters 4 and 5) were always measured by studying activity elicited by a trained stimulus, in comparison with an untrained stimulus, both presented in the absence of attention.

\section{Insights from the neurophysiology of selective attention, relevant for visual skill learning}

In the view we have taken of visual perceptual learning, we started from the hypothesis of an intimate relationship between attention and skill learning. The main function of attention as proposed by Reynolds and colleagues (2000) is to enhance the processing of weak stimuli. They proposed a contrast gain model or input gain model, in which attention selectively enhances weak inputs. In contrast, McAdams and Maunsell (1999) proposed a response gain or output gain model that predicts maximal enhancement taking place for maximized neuronal responses. Reynolds, Pasternak and Desimone (2000) explored their idea of input gain by recording from neurons whose receptive fields were stimulated by stimuli with a range of luminance contrasts, under attended and unattended conditions. They found that attention selectively increased activations for contrast at or below detection threshold, thereby selectively improving stimulus' detectability for weak stimuli, which is in line with the input gain model. The input gain model has found additional support in a series of studies using com- 
peting stimuli in the RF (Reynolds et al., 1999; Treue and Martinez Trujillo, 1999; Luck et al., 2000; Chelazzi et al., 2001), and in a number of studies pointing to Gamma coherence as a vehicle to bias processing (increased sensitivity) towards attended target stimuli (Fries et al., 2001; Buschman and Miller, 2007; Fries et al., 2008; Buschman and Miller, 2010). In the context of visual skill learning, one tantalizing prediction is that upon completion of visual skill learning with stimuli spanning a range of contrasts, stimuli at low to mid-range contrasts presented outside the focus of attention might yield an automatically enhanced neural response, as if the stimuli were being attended. According to this hypothesis, skill learning would lead to an automated incorporation of the attentional effect in the response to trained stimuli, and the response to trained stimuli would show input gain (compared to a baseline of untrained stimuli). Skill learning would become visible as a form of 'frozen attention'.

\section{Testing the link between attention and visual skill learning}

The learning paradigm we used in Chapter 5 gave us the opportunity to directly assess the contribution of attention to perceptual learning, as we tested training effects in the fMRI experiments for stimuli with a large range of contrasts. Specifically, the 'frozen attention' hypothesis could be tested by comparing fMRI responses to grating stimuli shown at various contrasts that had been subjected to visual skill learning, to fMRI responses to stimuli that had not been subjected to learning. Furthermore, the design of experiments in Chapters 4 and 5 included a condition of passive exposure and an attentive learning condition, in order to isolate effects of attention from effects of exposure and feedback.

\section{Thesis overview}

We have used a multidisciplinary approach (involving psychophysics, TMS and $\mathrm{fMRI}$ ) to address four fundamental questions in the domain of visual skill learning. In Chapter 2, we used a psychophysical interference paradigm to test the effect of overlap between neuronal populations and the time-interval between two interfering orientation discrimination tasks upon the magnitude of resulting behavioural interference. These experiments were designed to test hypotheses related to the concept of consolidation. In related experiments in Chapter 3, we used a TMS interference paradigm to test for functional indications of plasticity and offline activity in human low-level visual cortex. In Chapter 4 MVPA was employed to study the existence of long-term memory traces in primary visual cortex (V1), 90 days after the conclusion of an orientation discrimination training paradigm. Chapter 5 contains a longitudinal fMRI paradigm, designed to test the time-course of plasticity in multiple visual areas to distinguish RHT and LLT. Moreover, the paradigm was designed to test the concept of learning being a form of frozen attention. Participants were trained on an orientation 


\section{Introduction}

discrimination task, in which orientation differences were kept constant and performance was measured using contrast thresholds. Subsequently several fMRI scans were performed before, during and at the end of training when asymptotic performance was reached. Finally, Chapter 6 contains a summary and discussion of the experiments presented in this thesis and will end with an outline of perspectives for future research.

\section{References}

Aberg KC, Herzog MH (2010) Does perceptual learning suffer from retrograde interference? PLoS One 5:e14161.

Agranoff BW, Davis RE, Brink JJ (1965) Memory fixation in the goldfish. Proc Natl Acad Sci U S A 54:788-793.

Ahissar M, Hochstein S (1993) Attentional control of early perceptual learning. Proc Natl Acad Sci U S A 90:5718-5722.

Ahissar M, Hochstein S (1996) Learning pop-out detection: specificities to stimulus characteristics. Vision Res 36:3487-3500.

Ahissar M, Hochstein S (1997) Task difficulty and the specificity of perceptual learning. Nature 387:401-406.

Ahissar M, Hochstein S (2002) View from the top: hierarchies and reverse hierarchies in the visual system. Neuron 36:791-804.

Ahissar M, Hochstein S (2004) The reverse hierarchy theory of visual perceptual learning. Trends Cogn Sci 8:457-464.

Ahissar M, Laiwand R, Hochstein S (2001) Attentional demands following perceptual skill training. Psychol Sci 12:56-62.

Ahissar M, Laiwand R, Kozminsky G, Hochstein S (1998) Learning pop-out detection: building representations for conflicting target-distractor relationships. Vision Res 38:3095-3107.

Ahissar M, Nahum M, Nelken I, Hochstein S (2009) Reverse hierarchies and sensory learning. Philos Trans R Soc Lond B Biol Sci 364:285-299.

Albert NB, Robertson EM, Miall RC (2009a) The resting human brain and motor learning. Curr Biol 19:1023-1027.

Albert NB, Robertson EM, Mehta P, Miall RC (2009b) Resting state networks and memory consolidation. Commun Integr Biol 2:530-532.

Arckens L, Van Der Gucht E, Eysel UT, Orban GA, Vandesande F (2000) Investigation of cortical reorganization in area 17 and nine extrastriate visual areas through the detection of changes in immediate early gene expression as induced by retinal lesions. J Comp Neurol 425:531-544.

Atkinson RC, Shiffrin RM, eds (1968) Human memory: A proposed system and its control processes. London: Acadamic Press.

Ball K, Sekuler R (1987) Direction-specific improvement in motion discrimination. Vision Res 27:953-965.

Blasdel GG, Mitchell DE, Muir DW, Pettigrew JD (1977) A physiological and behavioural study in cats of the effect of early visual experience with contours of a single orientation. $J$ 
Physiol 265:615-636.

Bourtchouladze R, Abel T, Berman N, Gordon R, Lapidus K, Kandel ER (1998) Different training procedures recruit either one or two critical periods for contextual memory consolidation, each of which requires protein synthesis and PKA. Learn Mem 5:365-374. Brashers-Krug T, Shadmehr R, Bizzi E (1996) Consolidation in human motor memory. Nature 382:252-255.

Buschman TJ, Miller EK (2007) Top-down versus bottom-up control of attention in the prefrontal and posterior parietal cortices. Science 315:1860-1862.

Buschman TJ, Miller EK (2010) Shifting the spotlight of attention: evidence for discrete computations in cognition. Front Hum Neurosci 4:194.

Butefisch CM, Davis BC, Wise SP, Sawaki L, Kopylev L, Classen J, Cohen LG (2000)

Mechanisms of use-dependent plasticity in the human motor cortex. Proc Natl Acad Sci U S A 97:3661-3665.

Caithness G, Osu R, Bays P, Chase H, Klassen J, Kawato M, Wolpert DM, Flanagan JR (2004) Failure to consolidate the consolidation theory of learning for sensorimotor adaptation tasks. J Neurosci 24:8662-8671.

Censor N, Bonneh Y, Arieli A, Sagi D (2009) Early-vision brain responses which predict human visual segmentation and learning. J Vis 9:12 11-19.

Chelazzi L, Miller EK, Duncan J, Desimone R (2001) Responses of neurons in macaque area V4 during memory-guided visual search. Cereb Cortex 11:761-772.

Chung ST, Levi DM, Li RW (2006) Learning to identify contrast-defined letters in peripheral vision. Vision Res 46:1038-1047.

Craik FIM, Lockhart RS (1972) Levels of processing: A framework for memory research. Journal of Verbal Learning and Verbal Behavior 11:671-684.

Crist RE, Kapadia MK, Westheimer G, Gilbert CD (1997) Perceptual learning of spatial localization: specificity for orientation, position, and context. J Neurophysiol 78:2889-2894. Daselaar SM, Huijbers W, de Jonge M, Goltstein PM, Pennartz CM (2010) Experience-dependent alterations in conscious resting state activity following perceptuomotor learning. Neurobiol Learn Mem 93:422-427.

De Weerd P, Pinaud R, Bertini G (2006) Plasticity in V1 induced by perceptual learning. In: Plasticity in the visual system: From genes to circuits. (Pinaud R, Tremere L, De Weerd P, eds). New York: Springer.

Dinse HR, Kleibel N, Kalisch T, Ragert P, Wilimzig C, Tegenthoff M (2006) Tactile coactivation resets age-related decline of human tactile discrimination. Ann Neurol 60:88-94.

Dudai Y (2004) The neurobiology of consolidations, or, how stable is the engram? Annu Rev Psychol 55:51-86. Duncan CP (1949) The retroactive effect of electroshock on learning. J Comp Physiol Psychol 42:32-44.

Fahle M, Edelman S (1993) Long-term learning in vernier acuity: effects of stimulus orientation, range and of feedback. Vision Res 33:397-412.

Fiorentini A, Berardi N (1980) Perceptual learning specific for orientation and spatial frequency. Nature 287:43-44.

Fiorentini A, Berardi N (1981) Learning in grating waveform discrimination: specificity for orientation and spatial frequency. Vision Res 21:1149-1158. 


\section{Introduction}

Franko E, Seitz AR, Vogels R (2010) Dissociable neural effects of long-term stimulus-reward pairing in macaque visual cortex. J Cogn Neurosci 22:1425-1439.

Frenkel MY, Sawtell NB, Diogo AC, Yoon B, Neve RL, Bear MF (2006) Instructive effect of visual experience in mouse visual cortex. Neuron 51:339-349.

Fries P, Reynolds JH, Rorie AE, Desimone R (2001) Modulation of oscillatory neuronal synchronization by selective visual attention. Science 291:1560-1563.

Fries P, Womelsdorf T, Oostenveld R, Desimone R (2008) The effects of visual stimulation and selective visual attention on rhythmic neuronal synchronization in macaque area V4. J Neurosci 28:4823-4835.

Furmanski CS, Engel SA (2000) Perceptual learning in object recognition: object specificity and size invariance. Vision Res 40:473-484.

Gervan P, Kovacs I (2010) Two phases of offline learning in contour integration. J Vis 10:24. Ghose GM, Yang T, Maunsell JH (2002) Physiological correlates of perceptual learning in monkey V1 and V2. J Neurophysiol 87:1867-1888.

Godde B, Stauffenberg B, Spengler F, Dinse HR (2000) Tactile coactivation-induced changes in spatial discrimination performance. J Neurosci 20:1597-1604.

Goedert KM, Willingham DB (2002) Patterns of interference in sequence learning and prism adaptation inconsistent with the consolidation hypothesis. Learn Mem 9:279-292.

Gold J, Bennett PJ, Sekuler AB (1999) Signal but not noise changes with perceptual learning. Nature 402:176-178.

Gold J, Sekuler AB, Bennett PJ (2004) Characterizing perceptual learning with external noise. Cognitive Science 2004:167-207.

Gutnisky DA, Hansen BJ, Iliescu BF, Dragoi V (2009) Attention alters visual plasticity during exposure-based learning. Curr Biol 19:555-560.

Harlow HF, Zimmermann RR (1959) Affectional responses in the infant monkey; orphaned baby monkeys develop a strong and persistent attachment to inanimate surrogate mothers. Science 130:421-432.

Helmholtz H (1910) Treatise on Physiological Optics. New York: Dover. Herzog MH, Fahle $M$ (1997) The role of feedback in learning a vernier discrimination task. Vision Res 37:2133-2141.

Hubel DH, Wiesel TN (1963) Receptive Fields of Cells in Striate Cortex of Very Young, Visually Inexperienced Kittens. J Neurophysiol 26:994-1002.

Hussain Z, Sekuler AB, Bennett PJ (2009) How much practice is needed to produce perceptual learning? Vision Res 49:2624-2634.

Jeter PE, Dosher BA, Petrov A, Lu ZL (2009) Task precision at transfer determines specificity of perceptual learning. J Vis 9:1 1-13.

Joseph R (1996) Limbic language, social-emotional intelligence, development and attachment. Baltimore: Williams \& Wilkins.

Joseph R (1999) Environmental influences on neural plasticity, the limbic system, emotional development and attachment: a review. Child Psychiatry Hum Dev 29:189-208.

Kaas JH, Krubitzer LA, Chino YM, Langston AL, Polley EH, Blair N (1990) Reorganization of retinotopic cortical maps in adult mammals after lesions of the retina. Science 248:229-231.

Kandel ER, Kupfermann I, Iversen S, eds (2000) Learning and Memory. 
Karni A (1996) The acquisition of perceptual and motor skills: a memory system in the adult human cortex. Brain Res Cogn Brain Res 5:39-48.

Karni A, Sagi D (1991) Where practice makes perfect in texture discrimination: evidence for primary visual cortex plasticity. Proc Natl Acad Sci U S A 88:4966-4970.

Karni A, Sagi D (1993) The time course of learning a visual skill. Nature 365:250-252.

Karni A, Bertini G (1997) Learning perceptual skills: behavioral probes into adult cortical plasticity. Curr Opin Neurobiol 7:530-535.

Karni A, Tanne D, Rubenstein BS, Askenasy JJ, Sagi D (1994) Dependence on REM sleep of overnight improvement of a perceptual skill. Science 265:679-682.

Karni A, Meyer G, Rey-Hipolito C, Jezzard P, Adams MM, Turner R, Ungerleider LG (1998) The acquisition of skilled motor performance: fast and slow experience-driven changes in primary motor cortex. Proc Natl Acad Sci U S A 95:861-868.

Konorski J (1948) Conditioned reflexes and neuron organization. Cambridge: Cambridge University Press.

Korman M, Raz N, Flash T, Karni A (2003) Multiple shifts in the representation of a motor sequence during the acquisition of skilled performance. Proc Natl Acad Sci U S A

100:12492-12497.

Law CT, Gold JI (2008) Neural correlates of perceptual learning in a sensory-motor, but not a sensory, cortical area. Nat Neurosci 11:505-513.

Lu ZL, Hua T, Huang CB, Zhou Y, Dosher BA (2011) Visual perceptual learning. Neurobiol Learn Mem 95:145-151.

Luck SJ, Woodman GF, Vogel EK (2000) Event-related potential studies of attention. Trends Cogn Sci 4:432-440.

Luft AR, Buitrago MM, Ringer T, Dichgans J, Schulz JB (2004) Motor skill learning depends on protein synthesis in motor cortex after training. J Neurosci 24:6515-6520. Lupien SJ, Gaudreau S, Tchiteya BM, Maheu F, Sharma S, Nair NP, Hauger RL, McEwen BS, Meaney MJ (1997) Stress-induced declarative memory impairment in healthy elderly subjects: relationship to cortisol reactivity. J Clin Endocrinol Metab 82:2070-2075.

Mazoyer B, Houde O, Joliot M, Mellet E, Tzourio-Mazoyer N (2009) Regional cerebral blood flow increases during wakeful rest following cognitive training. Brain Res Bull 80:133-138.

McAdams CJ, Maunsell JH (1999) Effects of attention on the reliability of individual neurons in monkey visual cortex. Neuron 23:765-773.

McKee SP, Westheimer G (1978) Improvement in vernier acuity with practice. Percept Psychophys 24:258-262.

Mednick SC, Nakayama K, Cantero JL, Atienza M, Levin AA, Pathak N, Stickgold R (2002) The restorative effect of naps on perceptual deterioration. Nat Neurosci 5:677-681. Merzenich MM, Kaas JH, Wall J, Nelson RJ, Sur M, Felleman D (1983) Topographic reorganization of somatosensory cortical areas $3 \mathrm{~b}$ and 1 in adult monkeys following restricted deafferentation. Neuroscience 8:33-55.

Muellbacher W, Ziemann U, Wissel J, Dang N, Kofler M, Facchini S, Boroojerdi B, Poewe W, Hallett M (2002) Early consolidation in human primary motor cortex. Nature 415:640-644.

Nadel L, Moscovitch M (1997) Memory consolidation, retrograde amnesia and the hippocampal complex. Curr Opin Neurobiol 7:217-227. 


\section{Introduction}

Nadel L, Samsonovich A, Ryan L, Moscovitch M (2000) Multiple trace theory of human memory: computational, neuroimaging, and neuropsychological results. Hippocampus 10:352-368.

Poggio T, Fahle M, Edelman S (1992) Fast perceptual learning in visual hyperacuity. Science 256:1018-1021.

Pons TP, Garraghty PE, Mishkin M (1988) Lesion-induced plasticity in the second somatosensory cortex of adult macaques. Proc Natl Acad Sci U S A 85:5279-5281.

Pons TP, Garraghty PE, Ommaya AK, Kaas JH, Taub E, Mishkin M (1991) Massive cortical reorganization after sensory deafferentation in adult macaques. Science 252:1857-1860.

Raiguel S, Vogels R, Mysore SG, Orban GA (2006) Learning to see the difference specifically alters the most informative V4 neurons. J Neurosci 26:6589-6602.

Ramachandran VS, Braddick O (1973) Orientation-specific learning in stereopsis.

Perception 2:371-376.

Recanzone GH, Merzenich MM, Jenkins WM (1992) Frequency discrimination training engaging a restricted skin surface results in an emergence of a cutaneous response zone in cortical area 3a. J Neurophysiol 67:1057-1070.

Recanzone GH, Schreiner CE, Merzenich MM (1993) Plasticity in the frequency representation of primary auditory cortex following discrimination training in adult owl monkeys. J Neurosci 13:87-103.

Reed A, Riley J, Carraway R, Carrasco A, Perez C, Jakkamsetti V, Kilgard MP (2011) Cortical map plasticity improves learning but is not necessary for improved performance. Neuron 70:121-131.

Reynolds JH, Chelazzi L, Desimone R (1999) Competitive mechanisms subserve attention in macaque areas V2 and V4. J Neurosci 19:1736-1753.

Reynolds JH, Pasternak T, Desimone R (2000) Attention increases sensitivity of V4 neurons. Neuron 26:703-714.

Robertson EM (2004) Skill learning: putting procedural consolidation in context. Curr Biol 14:R1061-1063.

Robertson EM, Pascual-Leone A, Miall RC (2004) Current concepts in procedural consolidation. Nat Rev Neurosci 5:576-582.

Rubehn B, Bosman C, Oostenveld R, Fries P, Stieglitz T (2009) A MEMS-based flexible multichannel ECoG-electrode array. J Neural Eng 6:036003.

Sakai K, Kitaguchi K, Hikosaka O (2003) Chunking during human visuomotor sequence learning. Exp Brain Res 152:229-242.

Salvi RJ, Wang J, Ding D (2000) Auditory plasticity and hyperactivity following cochlear damage. Hear Res 147:261-274.

Schmid MC, Panagiotaropoulos T, Augath MA, Logothetis NK, Smirnakis SM (2009)

Visually driven activation in macaque areas $\mathrm{V} 2$ and $\mathrm{V} 3$ without input from the primary visual cortex. PLoS One 4:e5527.

Schoups A, Vogels R, Qian N, Orban G (2001) Practising orientation identification improves orientation coding in V1 neurons. Nature 412:549-553.

Schoups AA, Vogels R, Orban GA (1995) Human perceptual learning in identifying the oblique orientation: retinotopy, orientation specificity and monocularity. J Physiol 483 ( $\mathrm{Pt}$ 3):797-810. 
Seitz AR, Watanabe T (2003) Psychophysics: Is subliminal learning really passive? Nature 422:36.

Seitz AR, Yamagishi N, Werner B, Goda N, Kawato M, Watanabe T (2005) Task-specific disruption of perceptual learning. Proc Natl Acad Sci U S A 102:14895-14900.

Shadmehr R, Holcomb HH (1997) Neural correlates of motor memory consolidation.

Science 277:821-825.

Shiu LP, Pashler H (1992) Improvement in line orientation discrimination is retinally local but dependent on cognitive set. Percept Psychophys 52:582-588.

Sigman M, Gilbert CD (2000) Learning to find a shape. Nat Neurosci 3:264-269.

Smeets T, Otgaar H, Candel I, Wolf OT (2008) True or false? Memory is differentially affected by stress-induced cortisol elevations and sympathetic activity at consolidation and retrieval. Psychoneuroendocrinology 33:1378-1386.

Smirnakis SM, Brewer AA, Schmid MC, Tolias AS, Schuz A, Augath M, Inhoffen W, Wandell BA, Logothetis NK (2005) Lack of long-term cortical reorganization after macaque retinal lesions. Nature 435:300-307.

Spalding D (1873) Instinct, with original observations on young animals. In: MacMillan's Magazine, pp 282-293. Squire LR (1992) Memory and the hippocampus: a synthesis from findings with rats, monkeys, and humans. Psychol Rev 99:195-231.

Squire LR (2004) Memory systems of the brain: a brief history and current perspective. Neurobiol Learn Mem 82:171-177.

Squire LR, Alvarez P (1995) Retrograde amnesia and memory consolidation: a neurobiological perspective. Curr Opin Neurobiol 5:169-177.

Stickgold R, James L, Hobson JA (2000) Visual discrimination learning requires sleep after training. Nat Neurosci 3:1237-1238.

Treue S, Martinez Trujillo JC (1999) Feature-based attention influences motion processing gain in macaque visual cortex. Nature 399:575-579.

Walker MP, Stickgold R, Alsop D, Gaab N, Schlaug G (2005) Sleep-dependent motor memory plasticity in the human brain. Neuroscience 133:911-917.

Wanisch K, Wotjak CT, Engelmann M (2008) Long-lasting second stage of recognition memory consolidation in mice. Behav Brain Res 186:191-196.

Watanabe T, Nanez JE, Sasaki Y (2001) Perceptual learning without perception. Nature 413:844-848.

Watanabe T, Nanez JE, Sr., Koyama S, Mukai I, Liederman J, Sasaki Y (2002) Greater plasticity in lower-level than higher-level visual motion processing in a passive perceptual learning task. Nat Neurosci 5:1003-1009.

Weiss Y, Edelman S, Fahle M (1993) Models of Perceptual Learning in Vernier Hyperacuity. Neural Computation 5:695-718.

Westheimer G, Crist RE, Gorski L, Gilbert CD (2001) Configuration specificity in bisection acuity. Vision Res 41:1133-1138.

Xiao LQ, Zhang JY, Wang R, Klein SA, Levi DM, Yu C (2008) Complete transfer of perceptual learning across retinal locations enabled by double training. Curr Biol 18:1922-1926.

Yotsumoto Y, Watanabe T, Sasaki Y (2008) Different dynamics of performance and brain activation in the time course of perceptual learning. Neuron 57:827-833. 


\section{Introduction}

Yotsumoto Y, Sasaki Y, Chan P, Vasios CE, Bonmassar G, Ito N, Nanez JE, Sr., Shimojo S, Watanabe $T$ (2009) Location-specific cortical activation changes during sleep after training for perceptual learning. Curr Biol 19:1278-1282.

Zhang J, Kourtzi Z (2010) Learning-dependent plasticity with and without training in the human brain. Proc Natl Acad Sci U S A 107:13503-13508.

Zola-Morgan SM, Squire LR (1990) The primate hippocampal formation: evidence for a time-limited role in memory storage. Science 250:288-290. 


\section{Time-Limited Consolidation and Task Interference: No Direct Link}

based on: Been, M., Jans, B., De Weerd ,P., Time-Limited Consolidation and Task Interference: No Direct Link. Journal Of Neuroscience, 31(42), 14944-14951. 


\section{Abstract}

Perceptual skills improve with daily practice (Fahle and Poggio, 2002; Fine and Jacobs, 2002). Practice induces plasticity in task-relevant brain regions during an "offline" consolidation period thought to last several hours, during which initially fragile memory traces become stable (Karni, 1996; Dudai, 2004). Impaired retention of a task if followed by training in another task is considered evidence for the instability of memory traces during consolidation (Dudai, 2004). However, it remains unknown when after training memory traces become stable and resistant against interference, where in the brain the neuronal mechanisms responsible for interference are localized, and how these mechanisms produce interference. Here, we show in human participants strong interference between two visual skill-learning tasks for surprisingly long time intervals between training periods (up to $24 \mathrm{~h}$ ). Interference occurred during asymptotic learning, but only when stimuli were similar between tasks. This supports a strong contribution to interference of low-level visual cortical areas (Karni and Bertini, 1997; Ahissar and Hochstein, 2004), where similar stimuli recruit overlapping neuronal populations. Our finding of stimulus-dependent and time-independent interference reveals a fundamental limit in cortical plasticity that constrains the simultaneous representation of multiple skills in a single neuronal population, rather than a time-limited consolidation process.

\section{Introduction}

Consolidation is classically defined as a several-hours-long, time-limited process of neuronal plasticity following a learning experience during which initially fragile memory traces become stabilized (Dudai, 2004). In the domain of skills, a form of long-term memory (LTM) (Kandel et al., 2000), the hypothesized fragility of implicit memories during consolidation predicts interference with traininginduced gains in behavioral expertise in a task if it is followed by training in another task within a restricted time interval (behavioral interference).

However, reports of behavioral interference have been inconsistent. It is not a universal finding (Aberg and Herzog, 2010), and in studies that did report interference, it was found for widely varying time intervals, from $0 \mathrm{~h}$ up to $4 \mathrm{~h}$ in some studies (Brashers-Krug et al., 1996; Shadmehr and Holcomb, 1997; Seitz et al., 2005), to $24 \mathrm{~h}$ up to a week in others (Goedert and Willingham, 2002; Caithness et al., 2004; Zhang et al., 2008). These findings raise fundamental questions about the notion of time-limited consolidation and its relation to behavioral interference.

Behavioral interference is frequently linked with the interruption of timelimited cellular consolidation processes (Dudai, 2004), but may also reflect overlap between neuronal populations involved in storing memory traces for different tasks (O'Reilly and Rudy, 2001). The latter factor may contribute to the variable results in prior studies, which have limited themselves to the very 
first session (Brashers-Krug et al., 1996; Shadmehr and Holcomb, 1997; Goedert and Willingham, 2002; Caithness et al., 2004) or first few sessions (Seitz et al., 2005; Zhang et al., 2008) of skill learning. During early learning, networks in high-level cortical regions orchestrate strategic choices, as well as attentional and motivational allocation in response to specific task demands, instructions, and context (Willingham, 1999; Hochstein and Ahissar, 2002; Doyon et al., 2003). Therefore, high-level networks involved in memory storage in two subsequent tasks may overlap or not, based on variables that are difficult to control. Consequently, opposite conclusions in similar studies of behavioral interference are reported (Seitz et al., 2005; Aberg and Herzog, 2010), although Hung and Seitz (2011) suggest an additional explanation involving eye-movement control and procedural divergences.

If overlap between neuronal populations contributing to memory traces yields behavioral interference, the time window of behavioral interference cannot simply depend on the time course of cellular plastic processes occurring if a task had been trained in isolation. Instead, behavioral interference may depend strongly on the success of a systems-level process by which memory traces that may overlap initially become segregated. Thus, behavioral interference may depend more on an often unpredictable overlap between memory traces at a population level than on the more predictable time course of known cellular plastic processes.

Because the contribution of representational overlap of memory traces to behavioral interference cannot be easily tested during early learning, we tested it during asymptotic learning. Here, memory storage recruits stimulus-specific populations in lower (sensory) cortical areas (Karni and Bertini, 1997; Hochstein and Ahissar, 2002; Ahissar and Hochstein, 2004), which permits testing the overlap between populations involved in memory storage on behavioral interference by simple stimulus manipulations.

\section{Materials and Methods}

\section{Participants}

All 29 (17 female, 12 male) participants had normal or corrected-to-normal visual acuity, gave written informed consent for all parts of the experiment, and were debriefed following completion of data acquisition. Participants were financially compensated for their participation. Participants were carefully selected on their motivation to participate in a long-term learning study. After their reaction to an advertisement, participants were invited for an interview in which general information was given about the experiment, and in which the importance of physical and mental fitness and sufficient sleep was emphasized. Participants were then sent home to carefully consider their decision and to recontact us within a few days to let us know their decision. 


\section{Stimuli, apparatus, and task}

Participants performed a visual orientation discrimination task (ODT) in which they indicated whether an oriented stimulus was tilted clockwise or counterclockwise with respect to a (never-shown) reference orientation of $135^{\circ}$. Stimuli consisted of Gabor patches ( 0.75 cycles $/{ }^{\circ}$ spatial frequency, $50 \%$ Michelson contrast) presented on a PC monitor (19 inch screen size, $60 \mathrm{~Hz}$ refresh rate, $1280 \times 1024$ pixel resolution) in one of the four quadrants of the visual field depending on condition $\left(3^{\circ}\right.$ diameter at $6^{\circ}$ eccentricity) while participants were seated in a dimly lit room. Average luminance of stimuli and of the gray background was $56 \mathrm{~cd} / \mathrm{m}^{2}$. Participants rested their head in a chin rest to stabilize head position relative to the stimulus monitor. Eye position was monitored with an infrared eye camera (60 Hz sampling rate Viewpoint Eye Tracker v.2.8.3, Arrington Research). A trial started with a blank gray screen, followed by presentation of a white fixation dot at the middle of the screen. In the first $500 \mathrm{~ms}$ of presentation of the fixation dot, participants were instructed to orient their eyes toward the fixation dot. This was followed by a $750 \mathrm{~ms}$ period in which accurate fixation would initiate another 250 ms period in which fixation was to be maintained, followed by a 500 ms presentation of the Gabor stimulus. Participants responded with their right index (left arrow key on keyboard) or middle finger (right arrow key on keyboard), respectively representing a counterclockwise or clockwise deviation from the reference orientation. The response window was 1000 ms. Participants received feedback on their response by a brief color change of the fixation dot (green for correct, red for incorrect). Stimulus presentation and response recording were performed using Cortex (v.5.9.6; $\mathrm{NIH}$ freeware for psychophysical and neurophysiological experimentation). Trials with eye position deviations beyond $1.5^{\circ}$ relative to the fixation dot were aborted and replaced by a new randomly generated trial. Trials in which no response was given were also randomly replaced.

\section{Training protocol}

Training was separated into two periods (Fig. 1B). During a first training period $(P 1)$, just-noticeable differences (JNDs) in orientation were determined at an oblique reference orientation $\left(135^{\circ}\right)$ in an Experimental $\left(E_{135}\right)$ and a Control quadrant $\left(\mathrm{C}_{135}\right)$. After a time interval $\mathrm{T}(0,0.5,1,3,6$, or $24 \mathrm{~h})$, additional training was given in a second training period (P2) at the same stimulus location in the experimental quadrant using reference orientations $105^{\circ}\left(E_{105}\right)$ and $165^{\circ}\left(E_{165}\right)$ (Fig. 1B). For each condition, learning curves were measured over 15 sessions. During each session, two blocks of four JNDs in orientation discrimination were determined. Within training periods, the quadrant order was counterbalanced across days/participants. For all but one time interval $(24 \mathrm{~h}$ ) between $\mathrm{P} 1$ and $\mathrm{P} 2$, the training periods were done on the same day. Although testing schedules differed between participants, they were all tested during daytime, and within participants, testing schedules were kept constant 
for the duration of the experiment. In the $24 \mathrm{~h}$ interval, P1 and P2 training took place on separate consecutive days, so two complete sessions were collected in a time span of a work week. In the experiment in which interference was compared for orientation differences of $90^{\circ}$ and $30^{\circ}$ between P1 and P2 reference orientations, the upper quadrants were the experimental quadrants in which interference was tested, and the control quadrant was in one of the lower quadrants. Within upper quadrants, the assignment of conditions was counterbalanced over participants, and the same held for the assignment of the control quadrant to the lower quadrants.

\section{Data and analyses}

JNDs were determined using a Wetherill and Levitt staircase that tracked an $84 \%$ correct performance. Measurements were terminated after 14 reversal points or when a total of 120 trials was reached (on average, 100 trials were performed per staircase), and computed as the geometric mean of the last 10 reversal points. During the initial session, the starting orientation difference was set at a large value. In later sessions, measurements started at the threshold level of the previous day. In total, one block of four staircases was performed in 20-30 min for each condition. All experiments consisted of four blocks, except for the experiment that included a quadrant in which P2 training was performed at a reference orientation orthogonal to the reference used for P1 training. In that experiment, daily sessions consisted of six or seven blocks, the latter case corresponding to "double" training (see Fig. 6A). All analyses in the current paper were performed on JNDs resulting from averaging per block of four staircases.

To quantify specific aspects of the learning curves, we used a piecewise nonlinear regression fitting procedure. The piecewise fit consisted of two parts: a nonlinear (exponential) decreasing function, and a monotonically decreasing linear function. The nonlinear part of the piecewise fit was constrained by taking the mean of the first eight staircases (two sessions) as its starting point. A constraint on the linear part of the piecewise fit was imposed by fixing the end point to the average of the last three sessions. Given these constraints, the shape parameters of the nonlinear and linear part and the positioning of the transition between the nonlinear and linear part along the learning curve were algorithmically optimized to best fit the data. The position along the learning curve of the transition point from exponentially to linearly decreasing function after optimization is defined as the inflection point (IP), and estimates the beginning of asymptotic learning.

\section{Results}

This study was designed to investigate the effect of overlap between the neuronal populations that are likely to be involved in memory formation for two 
tasks upon behavioral interference between the tasks. In both tasks, we tested perceptual skill learning in an orientation discrimination paradigm (see Materials and Methods), in which participants judged the clockwise or anticlockwise deviation of a Gabor stimulus from a reference orientation (Fig. 1A,B) and were trained until they reached asymptotic learning. Prior research shows that extensive training in orientation discrimination leads to large gains in performance (Vogels, 1990; Schoups et al., 1995) and to changes in orientation tuning in V1 and V4 neurons (Schoups et al., 2001; Raiguel et al., 2006). Tuning curves are most discriminative for orientation on their flanks, in $\mathrm{V} 1$ at $\sim 15^{\circ}$ from the preferred orientation (Fig. 1C,D), and training-induced steepening of flanks overlapping the trained orientation has been proposed to underlie performance enhancements in orientation discrimination (Schoups et al., 2001; Raiguel et al., 2006). We tested whether training-induced performance gains in a first task would be decreased by training in a second task with stimuli chosen so that learning in the two tasks relied on steepening opposite tuning curve flanks of the same neurons. To that aim, we combined training in a first testing period (P1) at an oblique reference orientation with training in a second testing period (P2) at reference orientations deviating by $30^{\circ}$ clockwise or anticlockwise from oblique (Fig. 1D).

\section{Behavioral interference occurs during asymptotic learning and for all time intervals}

Figure $2 A$ shows the learning curves for $E_{135}$ and $C_{135}$ for all P1-P2 time intervals (five participants per interval, except for $\Delta 24 \mathrm{~h}$, where we had four participants). For $\Delta 0 \mathrm{~h}$, learning curves in the control quadrant showed fast learning in the first few sessions, followed by asymptotic leaning. There was little learning in the experimental quadrant, leading to strongly increased JNDs in experimental compared to control quadrant by the end of testing. Hence, performance enhancements induced by $\mathrm{P} 1$ training at $\mathrm{E}_{135}$ were strongly interfered with by $P 2$ training at reference orientations $E_{105}$ and $E_{165}$. Strikingly, this pattern of results was present for all $\mathrm{P} 1-\mathrm{P} 2$ intervals. A repeated-measures ANOVA [Quadrant (2) x Interval (6) x Session (15)] showed significant effects for Session $(F(3.322,76.404)=14.412, p=0.001)$, Quadrant $(F(1,23)=$ $68.375, p=0.001)$, and Quadrant $\times$ Session $(F(5.588,128.521)=14.265, p=$ $0.001)$, confirming behavioral interference. The effects of Interval $(F(5,23)=$ 1.936, $p=0.126)$ and of the Interval $\times$ Session interaction $(F(16.610,76.404)$ $=1.098, \mathrm{p}=0.372$ ) were not significant, and therefore we pooled the dataset over intervals (Fig. 2B).

At the end of learning, there was a constant performance difference between $C_{135}$ and $E_{135}$, which was independent of the $P 1-P 2$ interval. Figure $2 C$ shows the difference between learning curve asymptotes in experimental and control quadrant averaged over the last eight sessions ( $\triangle$ Asymptote in In units). A one- 
A

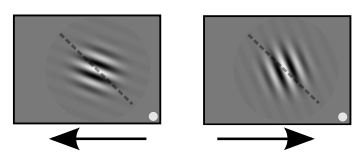

B

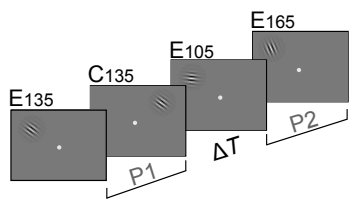

C

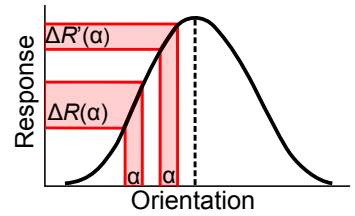

D

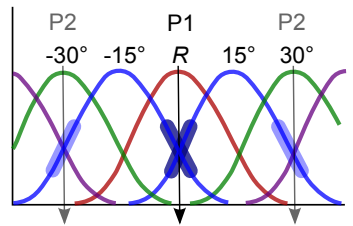

Figure 1. ODT and training-induced tuning changes in single neurons. A, Gabor stimuli and task. Participants fixated in the center of the screen while covertly attending the Gabor patch in an upper (left/right) quadrant of the visual field. Participants indicated the clockwise or anticlockwise deviation of the stimulus relative to a neverpresented reference (in this case $135^{\circ}$; dashed line shows reference for illustrative purposes) by pressing the right or left arrow key on a computer keyboard. Feedback was given by a change in color of the fixation dot (green, correct; red, incorrect). Trials with fixation errors $\left(>1.5^{\circ}\right.$ from fixation) were aborted (Arrington Research), and $84 \%$ correct staircase JNDs were determined (implemented in CORTEX 5.9.6, $\mathrm{NIH}$ freeware). B, Testing paradigm. Participants were tested in four conditions (4 JNDs of $\sim 100$ trials per condition). In training period P1 participants were trained at a $135^{\circ}$ reference orientation in upper left and right quadrant. One upper quadrant served as a control $\left(C_{135}\right)$. The other was the experimental quadrant $\left(E_{135}\right)$, where P1 training after a variable time interval of rest $(\Delta T)$ was followed by training in a second period $\mathrm{P} 2$ at reference orientations $105^{\circ}\left(\mathrm{E}_{105}\right)$ and $165^{\circ}\left(\mathrm{E}_{165}\right)$. C, Tuning curve of an orientation selective cell is most discriminative at its flanks. A difference $\alpha$ between two orientations on the flanks of the tuning curve will yield a differential response $\Delta R(\alpha)$ that is larger than the differential response $\Delta R^{\prime}(\alpha)$ yielded by the same difference between two orientations closer to the neuron's preferred orientation (dashed line). D, Our experimental design is informed by prior research showing that extensive training in orientation discrimination invoking asymptotic performance (Vogels and Orban, 1985; Schoups et al., 1995) selectively modified flank slopes of tuning curves in V1 and V4 neurons (Schoups et al., 2001; Raiguel et al., 2006). As V1 neurons are more narrowly tuned to orientation (David et al., 2006), and are probably more crucial during final asymptotic performance, we used $\mathrm{V} 1$ tuning properties to predict conditions of behavioral interference. In a V1 population neurons with tuning curve peaks about $15^{\circ}$ away (blue curves) from a trained reference orientation $\mathrm{R}$ $\left(135^{\circ}\right)$ (red curve) sharpen their tuning curve flanks (fat dark blue line segments) overlapping with the reference orientation. Extrapolating this idea, we predicted that following up $\mathrm{P} 1$ training at the reference orientation $\mathrm{R}$ with subsequent, $\mathrm{P} 2$ training at $\mathrm{R} \pm 30^{\circ}$ (green curves) would cause interference with training at $\mathrm{R}$. P1 training at $\mathrm{R}$ and subsequent $\mathrm{P} 2$ training at $\mathrm{R} \pm 30^{\circ}$ both require plasticity in neurons with preferred orientations at $\mathrm{R} \pm 15^{\circ}, \mathrm{P} 1$ training should increase the slope of flanks in tuning curves overlapping with $\mathrm{R}$, and $\mathrm{P} 2$ training the slope of flanks overlapping with the $+30^{\circ}$ and $-30^{\circ}$ orientations (fat light blue line segments). We hypothesized that one requirement would counteract the other, leading to behavioral interference at $R$ [P2 training is also expected to lead to steepening of flanks of $\pm 45^{\circ}$ neurons' tuning curves (in purple)]. 

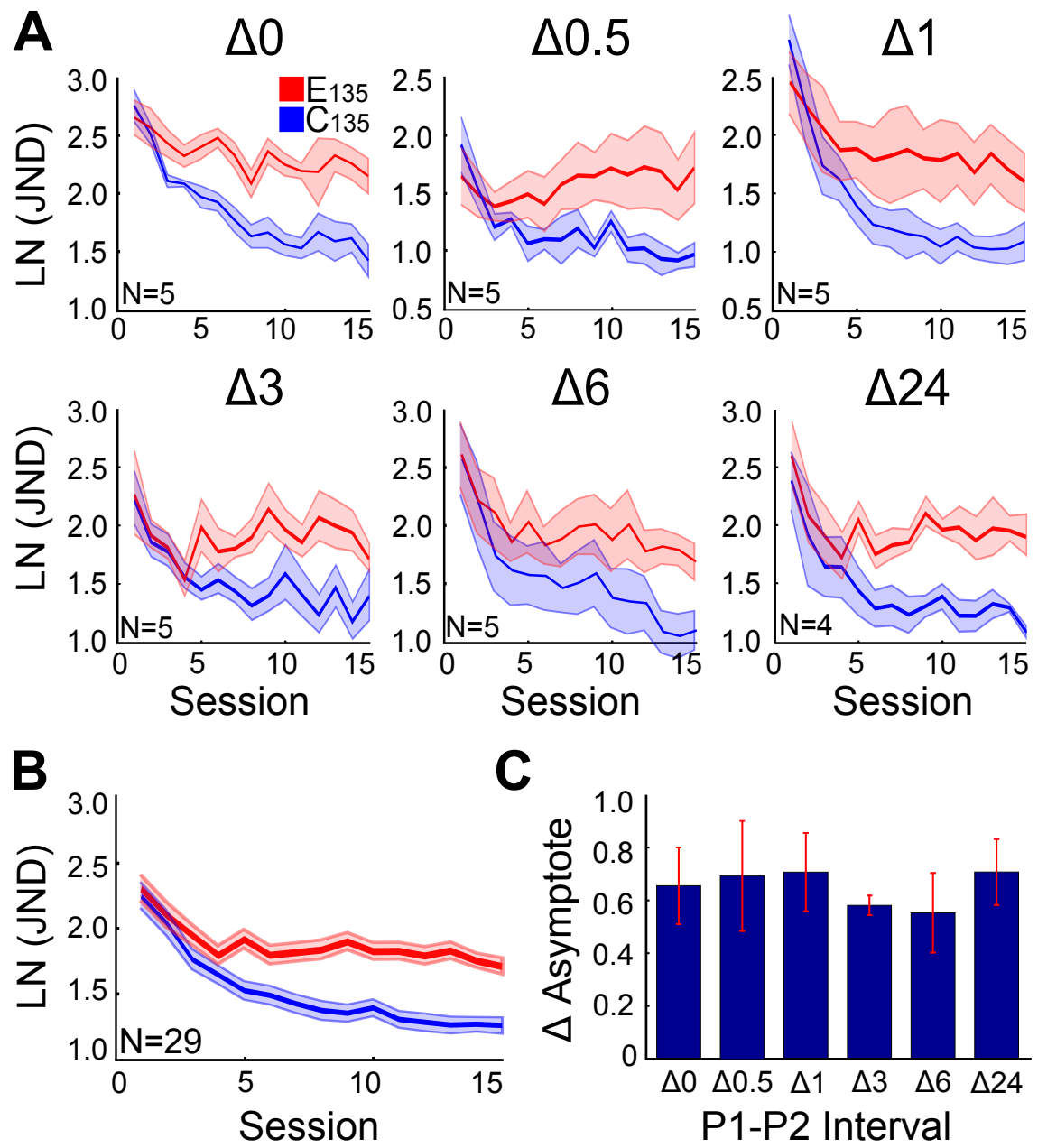

Figure 2. Behavioral interference during asymptotic learning. A, Interference for six different intervals between training period 1 (P1) and training period 2 (P2) $(\Delta 0-\Delta 24$ h). Each panel (corresponding to one interval) shows the natural logarithm of the just noticeable difference [LN(JND)] plotted as a function of session in experimental quadrant (red line) and control quadrant (blue line). For all intervals except the $24 \mathrm{~h}$ interval, P1 and P2 training were completed on the same day. In the $\Delta 24 \mathrm{~h}$ condition, $\mathrm{P} 1$ and $\mathrm{P} 2$ training were alternated from day to day, and a session therefore took $2 \mathrm{~d}$ (testing took $30 \mathrm{~d}$ ). B, Data averaged over P1-P2 intervals. C, Difference between learning curve asymptotes ( $\triangle$ Asymptote in In units) in experimental and control quadrant averaged over the last eight sessions. A one-way ANOVA showed that there was no significant difference in the size of $\triangle$ Asymptote between intervals $(F(5,23)=0.22, p=0.951)$. Shaded red and blue regions and error bars are standard errors; numbers in top right corner represent $\mathrm{N}$ participants (conventions are similar for all figures). 
way ANOVA showed that there was no significant difference in the magnitude of $\Delta$ Asymptote between intervals $(F(5,23)=0.22, p=0.951)$.

Further, in Figure 3 we show that interference started at asymptotic learning. Figure $3 \mathrm{~A}$ gives an illustration of the procedure to determine the beginning of asymptotic learning for the control condition at reference orientation $135^{\circ}\left(\mathrm{C}_{135}\right)$ in a single participant. First, the beginning of asymptotic learning was defined as the IP of the $C_{135}$ learning curve by using a piecewise nonlinear regression fitting procedure (see Materials and Methods). Then a comparison was made of the average inflection point per P1-P2 interval with the average session in the course of learning at which the first significant interference (FSI) is observed (averaged over subjects for each of the six P1-P2 intervals). We found that the inflection point of the learning curve for $C_{135}$, where asymptotic learning starts (session 7.4), was closely matched by the moment at which thresholds for $E_{135}$ first significantly exceed those for $C_{135}$ (session 6.5) ( $t=$ $-1.7399, \mathrm{df}=5, \mathrm{p}=0.1424$; paired t test on average $\mathrm{FSI}$ and IP per P1-P2 interval); this is in line with the idea that interference blocks learning when it is about to become asymptotic.

The finding that behavioral interference blocks asymptotic learning (Fig. 2B) suggests that it predominantly reflects interactions in early visual cortex (Karni and Bertini, 1997; Ahissar and Hochstein, 2004). The mechanism of interference may be related to $\mathrm{P} 1$ training at reference orientation $\mathrm{R}$ and subsequent $\mathrm{P} 2$ training at $\mathrm{R} \pm 30^{\circ}$ both requiring plasticity in neurons with preferred orientations at $R \pm 15^{\circ}$. In the $R \pm 15^{\circ}$ population (Fig. $1 D$, blue curves), $P 1$ training should increase the slope of flanks in tuning curves overlapping with $R$, and P2 training should increase the slope of flanks overlapping with the $+30^{\circ}$ and $30^{\circ}$ orientations. Our data suggest that one requirement counteracts the other, producing behavioral interference at R. Although we have based our explanatory model on V1 (Fig. 1D), contributions from other cortical areas with orientation selective neurons cannot be excluded.

The present study is the first to have described interference between two visual skill-learning tasks during prolonged, asymptotic learning. While we found interference to be time independent for time intervals between tasks of up to $24 \mathrm{~h}$, a prior study (Seitz et al., 2005) reported that behavioral interference between two Vernier tasks (after five daily sessions) disappeared with an interval between tasks of just $1 \mathrm{~h}$. Visual inspection of our data suggests that within the fast learning part of the learning curve, there could be a trend toward time-limited consolidation (Fig. 2A). To test this data trend, we ran a repeated-measures ANOVA on data from 10 subjects that were tested using the two P1-P2 intervals that are comparable to those used in the study of Seitz et al. (2005) [Condition $\left(E_{135}, C_{135}\right) \times$ Session (7) $\times$ P1-P2 Interval $(\Delta 0 \mathrm{~h}, \Delta 1$ h)] but failed to find a significant interaction between Condition and Interval $(F(1,8)=0.330, p=0.581)$. Thus, the reduction in the difference between $E_{135}$ and $C_{135}$ conditions in Figure $4 B$ compared to Figure $4 A$, which might suggest time-limited consolidation during early learning, was not confirmed by 

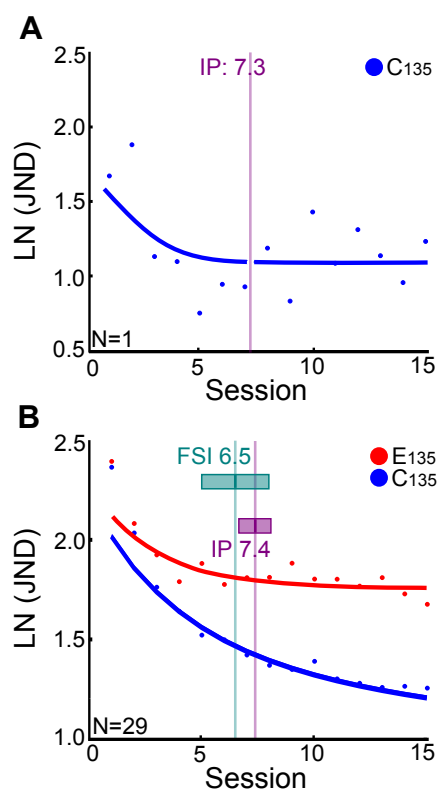

Figure 3. Significant interference starts at asymptotic learning. A, Illustration of the procedure to determine the beginning of asymptotic learning for the control condition at reference orientation $135^{\circ}\left(\mathrm{C}_{135}\right)$ in a single participant. The beginning of asymptotic learning was defined as the IP of the learning curve. To determine the IP, we used a piecewise nonlinear regression fitting procedure (see Materials and Methods). The results of optimized nonlinear and linear fitting as well as the optimized transition point (the IP; vertical gray line) are shown. The IP was determined for all intervals between the first (P1) and second (P2) training periods for the $\mathrm{C}_{135}$ condition, and the resulting IP (29 participants) was on average at session 7.4. B, Comparison of the average inflection point with the average session in the course of learning at which the FSI is observed. The figure shows the data for the control condition $\mathrm{C}_{135}$ (blue dots) and the experimental condition $\mathrm{E}_{135}$ (red dots). For both conditions, the data were averaged over the different P1-P2 intervals $(0,0.5,1,3,6,24 \mathrm{~h})$, as these experimental conditions did not significantly differ from each other. The fitted curves represent the average result of piecewise fitting for $C_{135}$ (blue) and $E_{135}$ (red). The vertical lines respectively mark the average session of FSI (green line) and average inflection point of the control condition $\mathrm{C}_{135}$ (IP, purple line) corresponding to the point at which initial fast learning is replaced with later asymptotic learning. While IP was based on estimates within each of 29 individuals, FSI was estimated in six groups of participants corresponding to the different P1-P2 delay conditions. The FSI was the first session in which paired-samples t-tests between the JNDs from $E_{135}$ and $\mathrm{C}_{135}$ showed a statistically significant difference. Averaged over the six P1-P2 delay conditions, this occurred at session 6.5. The two horizontal boxes show the size of the $95 \%$ confidence intervals, for both FSI and IP. Their overlap indicates that the distribution and the averages of the FSI and IP are not significantly different. In addition, a t-test between the FSI and IP for the six different P1-P2 intervals showed that the average FSI is not significantly different from the average IP $(t=1.7399, d f$ $=5, \mathrm{p}=0.1424$; paired). 
statistical testing. For completeness, we also made a comparison of $\Delta 0$ with all other P1-P2 Intervals in a combined repeated-measures ANOVA, and again the interaction between Condition and Interval was not significant $(F(5,23)=$ $0.374, p=0.861)$. Hence, we failed to reproduce the trend for time-limited consolidation during early learning reported by Seitz et al. (2005) in a different task.

\section{Behavioral interference is stimulus dependent}

During the training at the $+30^{\circ}$ and $-30^{\circ}$ reference orientations, there are neurons for which the tuning curve flanks overlap with these two references (deviating $\pm 45^{\circ}$ from R; purple curves in Fig. 1D) that are not stimulated by other stimuli. They are likely sufficient in number to permit normal orientation discrimination learning at the $+30^{\circ}$ and $-30^{\circ}$ axes. Accordingly, P2 training of $E_{105}$ and $E_{165}$ should slow down learning at $E_{135}$ induced by $P 1$ training, but $P 1$ training at $E_{135}$ should not affect $P 2$ training at $E_{105}$ and $E_{165}$. Figure $5 A$ shows exactly that result. Learning curves averaged over 29 subjects for conditions $E_{105}, E_{165}$, and $C_{135}$ overlapped completely. A repeated-measures ANOVA showed neither a main effect of Condition $\left(E_{105}, E_{165}, C_{135}\right)(F(2,56)=0.049$, $\mathrm{p}=0.952)$ nor an interaction between Condition and Session $(F(8.857,248)=$ $1.521, \mathrm{p}=0.142)$. Thus, the $\mathrm{E}_{105}$ and $\mathrm{E}_{165}$ training during $\mathrm{P} 2$ was unaffected by the preceding $E_{135}$ training because the $E_{135}$ training only affected half of the population contributing to the training in the $E_{105}$ and $E_{165}$ conditions. In the light of simulation studies indicating that on the order of 100 neurons permit normal orientation discrimination (Vogels, 1990), the normal training results at $E_{105}$ and $E_{165}$ are, in fact, unsurprising.

It could be argued, however, that JNDs in conditions $E_{105}$ and $E_{165}$ should be lower than those for $C_{135}$ because of the "oblique effect" in orientation discrimination (Appelle, 1972). If this were true, the lack of differences among these conditions might therefore still hide an effect of interference by the $135^{\circ}$ reference orientation on the $105^{\circ}$ and $165^{\circ}$ reference orientations. To investigate whether the performance on the P2 conditions $105^{\circ}$ or $165^{\circ}$ suffered from interference, we retested the five subjects that already had been trained on the $\Delta 0 \mathrm{~h}$ P1-P2 interval (Fig. 5A,B). This time, they were trained on the $105^{\circ}$ orientation in a naive visual quadrant $\left(E_{105}\right)$ where no other (potentially interfering) training on any other orientations had taken place. Performance in this condition was compared to new training at the $135^{\circ}$ reference orientation in another naive visual quadrant $\left(C_{135}\right)$. The naive quadrants used were the two lower quadrants.

The data confirm that the potential for behavioral interference decreases the less skill memories for the two tasks depend on the same neural population (O'Reilly and Rudy, 2001). We tested this further in seven participants by increasing the orientation difference between the reference orientation used during $\mathrm{P} 1$ training and the reference orientation used during $\mathrm{P} 2$ training. In a 

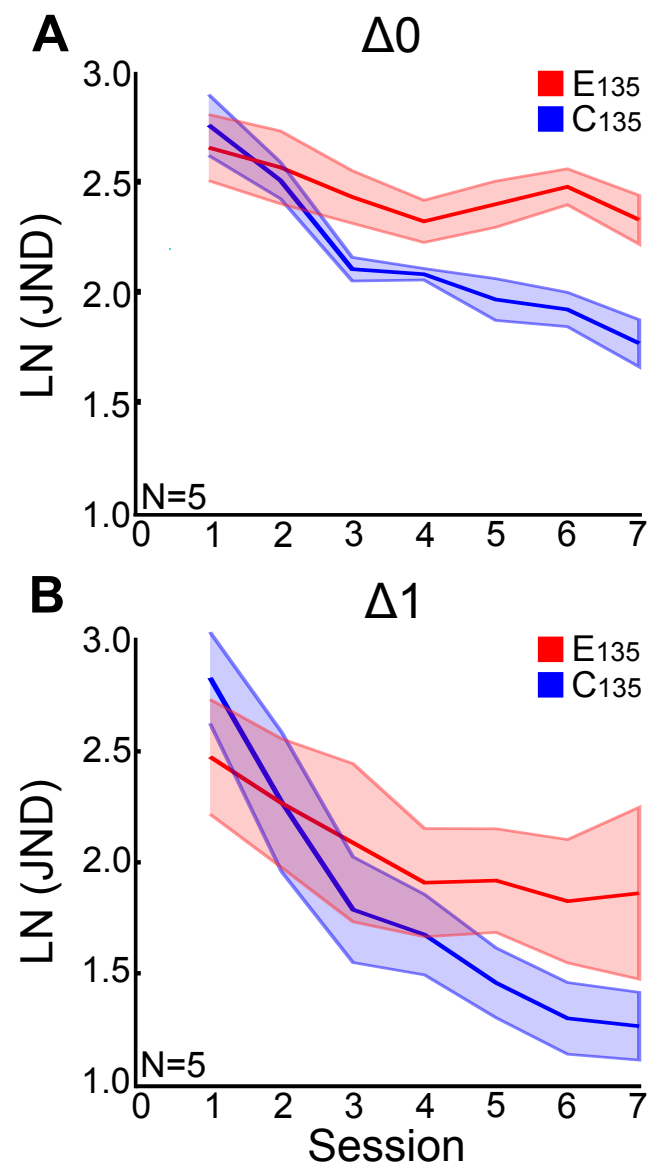

Figure 4. Comparing the fast learning part of the learning curves between the different P1-P2 intervals does not reveal time-limited consolidation. Here, a comparison of fast learning is shown at the $\Delta 0 \mathrm{~h}(\mathrm{~A})$ and $\Delta 1 \mathrm{~h}$ intervals $(\mathrm{B})$. $\mathrm{A}$, The first seven sessions for the $E_{135}$ and $C_{135}$ conditions in the $\Delta 0 \mathrm{~h}$ P1-P2 interval, up to the average IP. B, The first seven sessions for the $\Delta 1 \mathrm{~h}$ interval. These plots show the two intervals that are comparable to the intervals used in the paper of Seitz et al. (2005), who found time-dependent behavioral interference between two Vernier tasks during early learning. A repeated-measures ANOVA on our data [Condition $\left(E_{135}, C_{135}\right) x$ Session (7) $\times \mathrm{P} 1-\mathrm{P} 2$ Interval $(\Delta 0 \mathrm{~h}, \Delta 1 \mathrm{~h})]$ failed to find a significant interaction between Condition and Interval $(F(1,8)=0.330, p=0.581)$. Also, for redundancy, a comparison was made between $\Delta 0$ with all other P1-P2 intervals in a combined repeated-measures ANOVA; again, the interaction between Condition and Interval was not significant $(F(5,23)=0.374, \mathrm{p}=0.861)$. 


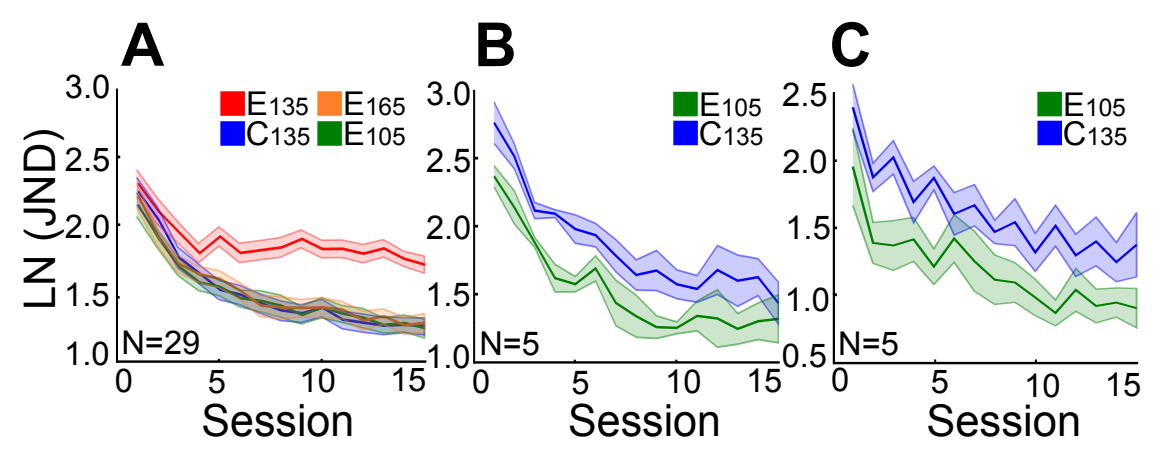

Figure 5. Stimulus dependency of behavioral interference. A, Interference is asymmetric. P2 training of $E_{105}$ and $E_{165}$ delays $P 1$ training of $E 135$, but not vice versa. Learning curves for $E_{105}$ and $E_{165}$ are indistinguishable from learning curves for $C_{135}$. $B$, Data of the five subjects from the $\Delta 0 \mathrm{~h} \mathrm{P1-P2}$ interval on the $\mathrm{C}_{135}$ and $\mathrm{E}_{105}$ conditions from the original experiment. $C$, Data for the same five subjects after extra training on new $\mathrm{C}_{135}$ and $\mathrm{E}_{105}$ conditions in the new, control experiment in which training at the $105^{\circ}$ reference orientation could not have suffered from interference. To analyze whether the $E_{105}$ performance was comparable in original (B) and control (C) experiments, a repeated-measures ANOVA [Experiment (original, control) $\times$ Condition $\left(C_{135}, E_{105}\right) \times$ Session (15)] was carried out. This analysis indicated that the Condition $\mathrm{x}===$ Experiment interaction was not significant $(\mathrm{F}(1,8)=1.501, \mathrm{p}=$ $0.255)$. Thus, the performance on the $E_{105}$ condition was similar in both experiments, thereby supporting the conclusion that training at the $105^{\circ}$ and $165^{\circ}$ reference orientations was not interfered with by training at the $135^{\circ}$ reference orientation in the same visual field position. 
first Experimental quadrant, we used a $90^{\circ}$ difference between the reference orientation during $\mathrm{P} 1$ training $\left(\mathrm{E} \mathrm{0}_{135}\right)$ and the reference during $\mathrm{P} 2$ training $\left(E 90_{45}\right)$. In a second Experimental quadrant, we used a $30^{\circ}$ difference between the $\mathrm{P} 1$ reference orientation $\left(E 30_{135}\right)$ and the $\mathrm{P} 2$ orientations $\left(E 30_{105}\right.$ and $\left.E 3_{165}\right)$. Upper left and right quadrants served as experimental quadrants and the control condition $\mathrm{C}_{135}$ was in one of the lower quadrants (Fig. 6A). Figure $6 \mathrm{~B}$ shows that learning curves from $\mathrm{P} 1$ training at $\mathrm{C}_{135}$ and $\mathrm{E} 90_{135}$ did not differ $(F(1,6)=0.237, \mathrm{p}=0.664$; compare gray and blue curves in Fig. $6 \mathrm{~B})$, while the JNDs of $\mathrm{C}_{135}$ and $\mathrm{E}_{30}{ }_{135}$ did differ $(\mathrm{F}(1,6)=12.788, \mathrm{p}=0.012$; compare red and blue curves in Fig. 6B).

To verify that the absence of interference was not confounded by the fact that four of the seven subjects tested were presented with only half of the trials during P2 training (a block of four JNDs at of $\mathrm{E90}_{45}$ ) compared to the number of trials given in P2 training in our standard design (four JNDs at $105^{\circ}$ and four JNDs at $165^{\circ}$ references), we performed an extra analyses on the data. The data of the same seven subjects were reanalyzed separating the subjects in two groups according to the number of trials they trained on in the P2 period. The results are plotted in Figure 6, C (eight JNDs of E9045) and D

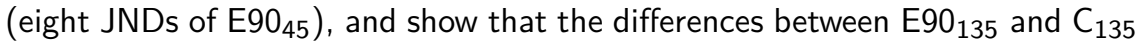
conditions are still negligible and did not increase when the amount of training on $\mathrm{E} 0_{45}$ was doubled in the P2 interval. Statistical evaluation confirmed that $\mathrm{P} 1$ training at the $135^{\circ}$ reference orientation is about equally unaffected by one or two blocks of $\mathrm{P} 2$ training at the orthogonal reference orientation (compare $\mathrm{E} 0_{135}$ with $\mathrm{C}_{135}$ in Fig. 6C,D). Specifically, a repeated-measures ANOVA [Double-Training (yes, no) $\times$ Condition $\left(C_{135}, E 90_{135}\right) \times$ Session (15)] showed that neither the main effect of Condition $(F(1,5)=0.95, p=0.770)$ nor the Condition $\times$ Double-Training interaction $(F(1,5)=2.946, p=0.147)$ were significant.

\section{Discussion}

We have studied factors contributing to LTM formation in a perceptual skilllearning task using a behavioral interference paradigm. Skill is one of many forms of LTM, which has been subdivided in two main classes: explicit (declarative) and implicit (nondeclarative) memory (Kandel et al., 2000; Squire, 2004). Declarative memory includes memory for facts and events, and implicit memory includes associative and nonassociative learning as well as motor and perceptual skill learning. Theories of LTM distinguish between the manipulation of information for storage (encoding), and storage itself (consolidation) (Atkinson and Shiffrin, 1968; Craik and Lockhart, 1972). The present study has focused on the processes that consolidate skill memories. Consolidation exists in both declarative (Squire, 1992; Squire and Alvarez, 1995) and implicit memory (Brashers-Krug et al., 1996; Karni, 1996; Karni et al., 1998; Robertson, 2004; Robertson et al., 2004), and refers to a process during which initially 

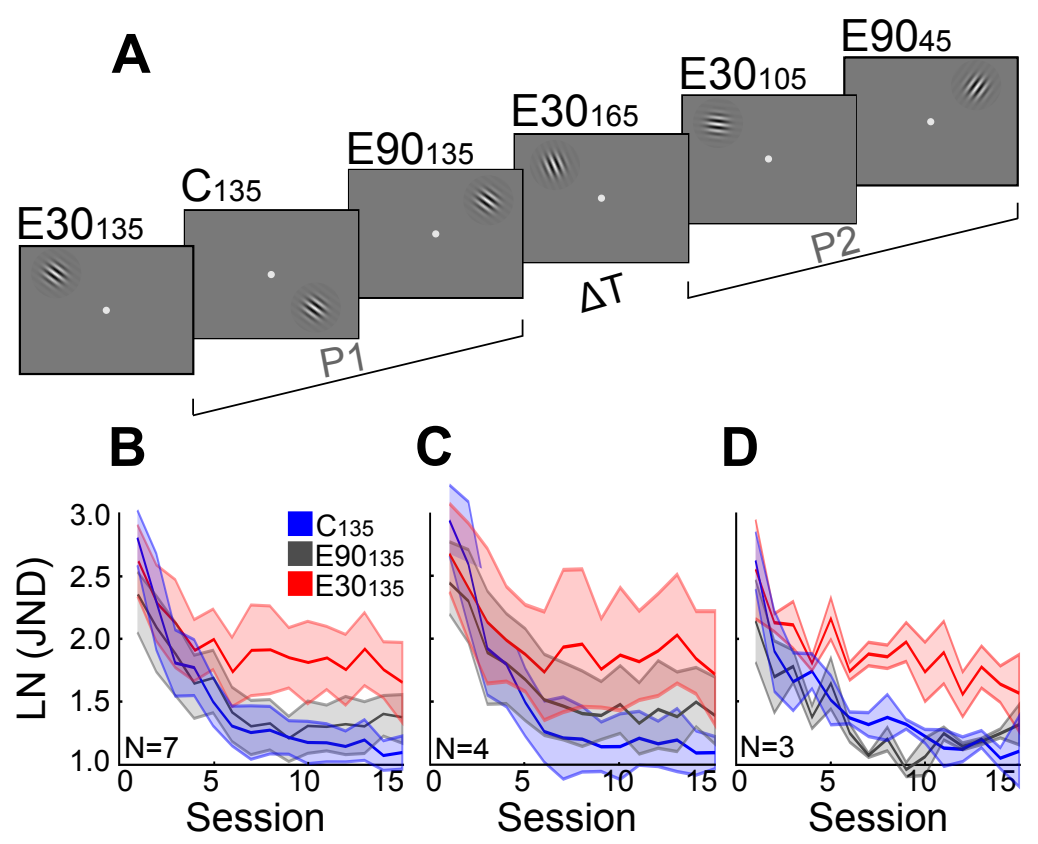

Figure 6. Double training does not cause behavioral interference. A, Experimental design used to replicate interfering effects of two blocks of training in $\mathrm{P} 2$ at reference orientations that differ by $30^{\circ}$ from $135^{\circ}$ ( $\mathrm{C}_{3} 0_{135}$ in $\mathrm{P} 1, \mathrm{E}_{30} 0_{105}$ and $\mathrm{E}_{30} 0_{165}$ in $\mathrm{P} 2$ ), and to compare these with interfering effects of a single block or two blocks in P2 at a reference orientation differing by $90^{\circ}$ from $135^{\circ}$ (E90 135 in $\mathrm{P} 1, \mathrm{E} 0_{45}$ in $\mathrm{P} 2$ ). Thus, while each stimulus display in Figure 6A corresponds to four JNDs, display $\mathrm{E} \mathrm{O}_{45}$ was tested in either four or eight JNDs. The number following " $E$ " refers to the orientation difference between reference orientations used during P1 and P2 training in the same location. $\mathrm{B}$, Training in orientation discrimination with orthogonal reference orientations during $\mathrm{P} 1$ and $\mathrm{P} 2$ does not cause behavioral interference $(\mathrm{N}=$ 7 ). The figure shows learning curves for $P 1$ training at the $135^{\circ}$ reference orientation in the control quadrant $\left(\mathrm{C}_{135}\right)$, for $\mathrm{P} 1$ training at the $135^{\circ}$ reference orientation in the experimental quadrant where $\mathrm{P} 2$ training was performed with an orthogonal $45^{\circ}$ reference $\left(E \mathrm{E0}_{135}\right)$ (either 4 or $8 \mathrm{JNDs}$ ), and for $\mathrm{P} 1$ training at the $135^{\circ}$ reference orientation in the experimental quadrant where $\mathrm{P} 2$ training was performed at $\pm 30^{\circ}$ references $\left(\mathrm{E}_{30} 0_{135}\right)$. Learning curves for $\mathrm{E} 0_{135}$ (dark gray) and $\mathrm{C}_{135}$ (blue) overlap. $\mathrm{C}$, Effect of training with a double block (8 JNDs) of P2 training at a $45^{\circ}$ reference (E9045) on learning rate in the preceding P1 training condition at $135^{\circ}\left(E_{90} 0_{135}\right)$. $\mathrm{D}$, Effect of training with a single block (4 JNDs) of P2 training at a $45^{\circ}$ reference $\left(E 90_{45}\right)$ in otherwise the same experiment. In both datasets, the $\mathrm{E} \mathrm{O}_{135}$ condition (dark gray line) is compared with the standard control condition $\mathrm{C}_{135}$ (blue line), and with the standard interference condition $\mathrm{E}^{3} 0_{135}$ (red line) in which $\mathrm{P} 1$ training at $135^{\circ}$ was followed by a block (4 JNDs) of P2 training at $105^{\circ}$ and a block at $165^{\circ}$. A repeated-measures ANOVA [Double-Training (yes, no) $\times$ Condition $\left(C_{135}, E E_{135}\right)$ $x$ Session (15)] showed that neither the main effect of Condition $(F(1,5)=0.95, p=$ $0.770)$ nor the Condition $\times$ Double-Training interaction $(F(1,5)=2.946, p=0.147)$ was significant. 
labile memory traces are transformed to stable memory traces. Because consolidation is thought to take place in a time-limited window extending beyond the experience by several hours (Dudai, 2004), behavioral interference is expected when a second experience falls within the consolidation window of a preceding experience. However, in our skill-learning study, we found that training in an orientation discrimination task could be interfered with by training in a second set of orientation discrimination tasks across a broad range of time intervals between training in $\mathrm{P} 1$ and $\mathrm{P} 2(0,0.5,1,3,6$, and $24 \mathrm{~h})$. Moreover, although varying the time interval between $\mathrm{P} 1$ and $\mathrm{P} 2$ did not modulate interference, stimulus similarity did, and increasing the stimulus orientation difference from $30^{\circ}$ to $90^{\circ}$ prevented interference.

To interpret our data, it is useful to distinguish cellular and systems-level processes contributing to LTM consolidation. At the cellular level, an experience triggers the expression of Immediate Early Genes (IEGs) whose protein products can act as transcription factors for Late Genes (LGs) involved in functional and structural modifications of synaptic strength between neurons (Dudai, 2004). Gene expression and protein synthesis associated with these initial cascades take place within minutes (IEGs) to hours (LGs) after neuronal stimulation, and disruption of these processes during or immediately after an experience interferes with memory consolidation (Duncan, 1949; Agranoff et al., 1965; Bütefisch et al., 2000; Muellbacher et al., 2002; Luft et al., 2004). Predictions of behavioral interference in skill-learning experiments seem to be based on this initial molecular cascade taking place over several hours following an experience (Brashers-Krug et al., 1996; Shadmehr and Holcomb, 1997; Seitz et al., 2005). However, cellular plasticity related to LTM formation may comprise multiple periods during waking (Bourtchouladze et al., 1998; Korman et al., 2003; Wanisch et al., 2008) and sleep (Karni et al., 1994; Stickgold et al., 2000; Gervan and Kovacs, 2010). Therefore, the notion of a single time-limited consolidation window cannot guide precise predictions about the time window in which behavioral interference would take place.

In addition, systems-level processes contribute to memory formation. Systemslevel considerations are an important aspect in theories of episodic memory formation in which a slow transfer of memory traces from hippocampus to cortex is proposed (Zola-Morgan and Squire, 1990; Squire and Alvarez, 1995), though this proposal is debated (Nadel and Moscovitch, 1997; Nadel et al., 2000). Strengthening of cortical memory traces is also thought to involve "replay," a term that refers to the reinstantiation of neural activity elicited by a prior experience. Replay is coordinated by low-frequency oscillatory activity (Jensen, 2006; Sirota et al., 2008), and has been shown in both hippocampal-based memory formation (Wilson and McNaughton, 1994; Hoffman and McNaughton, 2002) and skill-related memory formation (Stickgold et al., 2000; Yotsumoto et al., 2009). Another important systems-level factor is the requirement to segregate different memory traces. This is well recognized in the field of episodic memory formation (O'Reilly and Rudy, 2001), but has received less attention in the 
field of (perceptual) skill learning. During asymptotic perceptual learning, sensory neurons involved with acquisition during task performance with a specific stimulus are thought to contribute to memory formation (Karni and Bertini, 1997). This permitted predictions of specific stimulus conditions in which overlap of memory traces and behavioral interference would occur, and in which time-limited consolidation could be tested.

Because training induces slope changes in the flanks of orientation tuning curves with optimal orientations at $\pm 15^{\circ}$ from the trained reference $(R)$ orientation (Schoups et al., 2001), we predicted behavioral interference when P1 training at $\mathrm{R}$ was followed by $\mathrm{P} 2$ training at $\mathrm{R} \pm 15^{\circ}$ (see Fig. 1 legend). We confirmed this prediction for all six P1-P2 time intervals tested. The stimulus specificity of interference was confirmed by the finding that training with a $90^{\circ}$ difference between the reference orientations for P1 and P2 training, chosen to avoid overlap between memory traces, did not cause interference at the $1 \mathrm{~h}$ P1-P2 time interval. In addition, P2 training at reference orientations $105^{\circ}$ and $165^{\circ}$ was not affected by $\mathrm{P} 1$ training at the $135^{\circ}$ reference orientation. This may be due to half of the neuronal population associated with references $105^{\circ}$ and $165^{\circ}$ remaining unaffected by the training at the $135^{\circ}$ reference, which according to computational models (Vogels, 1990) is sufficient to permit normal orientation discrimination performance. Together, these findings show that the amount of overlap between populations involved in memory formation in low-level visual cortex (V1) is a highly determining factor for interference. Note that although our results suggest retrograde interference (P2 training affecting $P 1$ training) rather than anterograde interference ( $P 1$ training affecting $P 2$ training), the distinction between the two is difficult if the main factor leading to interference is stimulus-determined.

Our finding of equal behavioral interference for a range of time intervals is difficult to interpret in terms of the classical concept of time-limited consolidation. There is no doubt that time-dependent cellular processes take place in a behavioral interference experiment (likely in multiple periods). However, their interaction with systems-level factors makes the contributions of both factors difficult to separate. For short P1-P2 intervals, behavioral interference is likely to be related to interference with more short-lived cellular consolidation processes, but at longer time intervals (especially $24 \mathrm{~h}$ ), it may predominantly reflect the recruitment of a population of neurons that has become wired for a different task. However, regardless of the time interval between first and second training, overlap of memory traces is a precondition for interference to take place. Possibly, interference may be avoided by incorporating long periods without training (e.g., a week) after each pair of P1-P2 training sessions. This may permit segregation of memory traces through a slow competitive process in which memory traces may also become sparser. Even if this is true, the most straightforward interpretation of our data is that the behavioral interference observed in our study is stimulus-related and reflects a limit in the simultaneous 
representation of expertise on different skills in a single neuronal population $(\mathrm{Ni}$ and Maunsell, 2010).

This interpretation implies that memory traces remain highly malleable and far from immune for interference by other memory traces, even at asymptotic levels of expertise. In the domain of episodic memory formation, this idea has been proposed in the multiple-trace theory (Lewis, 1979; Nadel and Moscovitch, 2001). Here, previously consolidated episodic memories when retrieved become subject to reconsolidation (Lee et al., 2004; Lee, 2008), a process during which the original memory is modified. A related distinction has been proposed between active and inactive memory traces (Lewis, 1979; Caithness et al., 2004; $\mathrm{Xu}$ et al., 2009; Yang et al., 2009). In this framework, it is proposed that memory traces are active when they are formed, become inactive when they are not accessed, and are reactivated when retrieved (Nader et al., 2000; Nader, 2003). This reactivation returns the memory trace to a labile state in which it can be modified. Recent studies have shown that sensory experience leaves traces in the form of the size and number of synapses and dendritic spines ( $\mathrm{Xu}$ et al., 2009; Yang et al., 2009), and that the size of spines is related to the age of memories so that spine size may be a correlate of trace reactivation (Alvarez and Sabatini, 2007; Hofer et al., 2009). In the domain of skill learning, the malleability of memory traces provides a form of flexibility that can be advantageous. It permits efficient memory storage, because increases in expertise will lead to updating of a single memory rather than the creation of new memories for each level of increased skill, and replacement of expertise in an old skill with expertise required for a new skill.

In conclusion, we have provided the first full description of behavioral interference during asymptotic learning of two visual skill-learning tasks. We found that the extent of overlap between sensory neuronal populations stimulated in two competing tasks was the best predictor for the magnitude of behavioral interference, regardless of the state of memory formation for the first task when training in the second task was started. Hence, behavioral interference paradigms are not a sensitive approach to test the existence of specific time-limited consolidation processes. Instead, interference paradigms can help to identify limits in the capacity of neuronal subpopulations to simultaneously represent expertise for different skills during asymptotic learning.

\section{References}

Aberg KC, Herzog MH (2010) Does perceptual learning suffer from retrograde interference? PLoS One 5:e14161.

Agranoff BW, Davis RE, Brink JJ (1965) Memory fixation in the goldfish. Proc Natl Acad Sci U S A 54:788-793. Ahissar M, Hochstein S (2004) The reverse hierarchy theory of visual perceptual learning. Trends Cogn Sci 8:457-464.

Alvarez VA, Sabatini BL (2007) Anatomical and physiological plasticity of dendritic spines. Annu Rev Neurosci 30:79-97. 
Appelle S (1972) Perception and discrimination as a function of stimulus orientation-oblique effect in man and animals. Psychol Bull 78:266-278.

Atkinson RC, Shiffrin RM (1968) Human memory: a proposed system and its control processes. In: The psychology of learning and motivation (Spence KW, Spence JT, eds). London: Academic.

Bourtchouladze R, Abel T, Berman N, Gordon R, Lapidus K, Kandel ER (1998) Different training procedures recruit either one or two critical periods for contextual memory consolidation, each of which requires protein synthesis and PKA. Learn Mem 5:365-374. Brashers-Krug T, Shadmehr R, Bizzi E (1996) Consolidation in human motor memory. Nature 382:252-255.

Bütefisch CM, Davis BC, Wise SP, Sawaki L, Kopylev L, Classen J, Cohen LG (2000) Mechanisms of use-dependent plasticity in the human motor cortex. Proc Natl Acad Sci U S A 97:3661-3665.

Caithness G, Osu R, Bays P, Chase H, Klassen J, Kawato M, Wolpert DM, Flanagan JR (2004) Failure to consolidate the consolidation theory of learning for sensorimotor adaptation tasks. J Neurosci 24:8662-8671.

Craik FIM, Lockhart RS (1972) Levels of processing: a framework for memory research. J Verb Learn Verb Behav 11:671-684.

David SV, Hayden BY, Gallant JL (2006) Spectral receptive field properties explain shape selectivity in area V4. J Neurophysiol 96:3492-3505.

Doyon J, Penhune V, Ungerleider LG (2003) Distinct contribution of the cortico-striatal and cortico-cerebellar systems to motor skill learning. Neuropsychologia 41:252-262.

Dudai Y (2004) The neurobiology of consolidations, or, how stable is the engram? Annu Rev Psychol 55:51-86.

Duncan CP (1949) The retroactive effect of electroshock on learning. J Comp Physiol Psychol 42:32-44.

Fahle M, Poggio T (2002) Perceptual learning. Cambridge, MA: MIT Press.

Fine I, Jacobs RA (2002) Comparing perceptual learning across tasks: a review. J Vis 2:190-203.

Gervan P, Kovacs I (2010) Two phases of offline learning in contour integration. J Vis 10:24. Goedert KM, Willingham DB (2002) Patterns of interference in sequence learning and prism adaptation inconsistent with the consolidation hypothesis. Learn Mem 9:279-292.

Hochstein S, Ahissar M (2002) View from the top: hierarchies and reverse hierarchies in the visual system. Neuron 36:791-804.

Hofer SB, Mrsic-Flogel TD, Bonhoeffer T, Hulbener M (2009) Experience leaves a lasting structural trace in cortical circuits. Nature 457:313-317.

Hoffman KL, McNaughton BL (2002) Coordinated reactivation of distributed memory traces in primate neocortex. Science 297:2070-2073.

Hung SC, Seitz AR (2011) Retrograde interference in perceptual learning of a peripheral hyperacuity task. PLoS One 6:e24556.

Jensen O (2006) Maintenance of multiple working memory items by temporal segmentation. Neuroscience 139:237-249.

Kandel ER, Kupfermann I, Iversen S (2000) Learning and memory. In: Principles of neural science (Kandel ER, Schwartz JH, Jessell TM, eds), New York: McGraw-Hill. 


\section{Time-Limited Consolidation and Task Interference: No Direct Link}

Karni A (1996) The acquisition of perceptual and motor skills: a memory system in the adult human cortex. Brain Res Cogn Brain Res 5:39-48.

Karni A, Bertini G (1997) Learning perceptual skills: behavioral probes into adult cortical plasticity. Curr Opin Neurobiol 7:530-535.

Karni A, Tanne D, Rubenstein BS, Askenasy JJ, Sagi D (1994) Dependence on REM sleep of overnight improvement of a perceptual skill. Science 265:679-682.

Karni A, Meyer G, Rey-Hipolito C, Jezzard P, Adams MM, Turner R, Ungerleider LG (1998)

The acquisition of skilled motor performance: fast and slow experience-driven changes in primary motor cortex. Proc Natl Acad Sci U S A 95:861-868.

Korman M, Raz N, Flash T, Karni A (2003) Multiple shifts in the representation of a motor sequence during the acquisition of skilled performance. Proc Natl Acad Sci U S A

100:12492-12497.

Lee JL (2008) Memory reconsolidation mediates the strengthening of memories by additional learning. Nat Neurosci 11:1264-1266.

Lee JL, Everitt BJ, Thomas KL (2004) Independent cellular processes for hippocampalmemoryconsolidationandreconsolidation.Science304:839-843.

Lewis DJ (1979) Psychobiology of active and inactive memory. Psychol Bull 86:1054-1083. Luft AR, Buitrago MM, Ringer T, Dichgans J, Schulz JB (2004) Motor skill learning depends on protein synthesis in motor cortex after training. J Neurosci 24:6515-6520. Muellbacher W, Ziemann U, Wissel J, Dang N, Kofler M, Facchini S, Boroojerdi B, Poewe W, Hallett M (2002) Early consolidation in human primary motor cortex. Nature 415:640-644.

Nadel L, Moscovitch M (1997) Memory consolidation, retrograde amnesia and the hippocampal complex. Curr Opin Neurobiol 7:217-227.

Nadel L, Moscovitch M (2001) The hippocampal complex and long-term memory revisited. Trends Cogn Sci 5:228-230.

Nadel L, Samsonovich A, Ryan L, Moscovitch M (2000) Multiple trace theory of human memory: computational, neuroimaging, and neuropsychological results. Hippocampus 10:352-368.

Nader K (2003) Neuroscience: re-recording human memories. Nature 425:571-572.

Nader K, Schafe GE, LeDoux JE (2000) The labile nature of consolidation theory. Nat Rev Neurosci 1:216-219.

Ni AM, Maunsell JH (2010) Microstimulation reveals limits in detecting different signals from a local cortical region. Curr Biol 20:824-828.

O'Reilly RC, Rudy JW (2001) Conjunctive representations in learning and memory: principles of cortical and hippocampal function. Psychol Rev 108:311-345.

Raiguel S, Vogels R, Mysore SG, Orban GA (2006) Learning to see the difference specifically alters the most informative V4 neurons. J Neurosci 26:6589-6602.

Robertson EM (2004) Skill learning: putting procedural consolidation in context. Curr Biol 14:R1061-R1063.

Robertson EM, Pascual-Leone A, Miall RC (2004) Current concepts in procedural consolidation. Nat Rev Neurosci 5:576-582.

Schoups AA, Vogels R, Orban GA (1995) Human perceptual learning in identifying the oblique orientation: retinotopy, orientation specificity and monocularity. J Physiol 


\section{References}

483:797-810.

Schoups A, Vogels R, Qian N, Orban G (2001) Practising orientation identification improves orientation coding in V1 neurons. Nature 412:549-553.

Seitz AR, Yamagishi N, Werner B, Goda N, Kawato M, Watanabe T (2005) Task-specific disruption of perceptual learning. Proc Natl Acad Sci U S A 102:14895-14900.

Shadmehr R, Holcomb HH (1997) Neural correlates of motor memory consolidation. Science 277:821-825.

Sirota A,Montgomery S,Fujisawa S,Isomura Y,Zugaro M,Buzsat'ki G (2008) Entrainment of neocortical neurons and gamma oscillations by the hippocampal theta rhythm. Neuron 60:683-697.

Squire LR (1992) Memory and the hippocampus: a synthesis from findings with rats, monkeys, and humans. Psychol Rev 99:195-231.

Squire LR (2004) Memory systems of the brain: a brief history and current perspective. Neurobiol Learn Mem 82:171-177.

Squire LR, Alvarez P (1995) Retrograde amnesia and memory consolidation: a neurobiological perspective. Curr Opin Neurobiol 5:169-177.

Stickgold R, James L, Hobson JA (2000) Visual discrimination learning requires sleep after training. Nat Neurosci 3:1237-1238.

Vogels R (1990) Population coding of stimulus orientation by striate cortical cells. Biol Cybern 64:25-31.

Vogels R, Orban GA (1985) The effect of practice on the oblique effect in line orientation judgments. Vision Res 25:1679-1687.

Wanisch K, Wotjak CT, Engelmann M (2008) Long-lasting second stage of recognition memory consolidation in mice. Behav Brain Res 186: 191-196.

Willingham DB (1999) The neural basis of motor-skill learning. Curr Dir Psychol Sci 8:178-182.

Wilson MA, McNaughton BL (1994) Reactivation of hippocampal ensemble memories during sleep. Science 265:676-679.

Xu T, Yu X, Perlik AJ, Tobin WF, Zweig JA, Tennant K, Jones T, Zuo Y (2009) Rapid formation and selective stabilization of synapses for enduring motor memories. Nature 462:915-919.

Yang G, Pan F, Gan WB (2009) Stably maintained dendritic spines are associated with lifelong memories. Nature 462:920-924.

Yotsumoto Y, Sasaki Y, Chan P, Vasios CE, Bonmassar G, Ito N, Náñez JE Sr, Shimojo S, Watanabe T (2009) Location-specific cortical activation changes during sleep after training for perceptual learning. Curr Biol 19:1278-1282.

Zhang JY, Kuai SG, Xiao LQ, Klein SA, Levi DM, Yu C (2008) Stimulus coding rules for perceptual learning. PLoS Biol 6:e197.

Zola-Morgan SM, Squire LR (1990) The primate hippocampal formation: evidence for a time-limited role in memory storage. Science 250:288-290. 



\section{Post-training TMS of striate cortex disrupts consolidation early in visual skill learning}

based on: De Weerd, P., Reithler, J., van de Ven, V., Been, M., Jacobs C., \& Sack, A.T. Post-training TMS of striate cortex disrupts consolidation early in visual skill learning. Journal of Neuroscience, 32(6): 1981-1988. 


\section{Abstract}

Practice-induced improvements in skilled performance reflect "offline " consolidation processes extending beyond daily training sessions. According to visual learning theories, an early, fast learning phase driven by high-level areas is followed by a late, asymptotic learning phase driven by low-level, retinotopic areas when higher resolution is required. Thus, low-level areas would not contribute to learning and offline consolidation until late learning. Recent studies have challenged this notion, demonstrating modified responses to trained stimuli in primary visual cortex (V1) and offline activity after very limited training. However, the behavioral relevance of modified V1 activity for offline consolidation of visual skill memory in V1 after early training sessions remains unclear. Here, we used neuronavigated transcranial magnetic stimulation (TMS) directed to a trained retinotopic $\mathrm{V} 1$ location to test for behaviorally relevant consolidation in human low-level visual cortex. Applying TMS to the trained V1 location within $45 \mathrm{~min}$ of the first or second training session strongly interfered with learning, as measured by impaired performance the next day. The interference was conditional on task context and occurred only when training in the location targeted by TMS was followed by training in a second location before TMS. In this condition, high-level areas may become coupled to the second location and uncoupled from the previously trained low-level representation, thereby rendering consolidation vulnerable to interference. Our data show that, during the earliest phases of skill learning in the lowest-level visual areas, a behaviorally relevant form of consolidation exists of which the robustness is controlled by high-level, contextual factors.

\section{Introduction}

The neural mechanisms driving the remarkable improvements in performance after prolonged skill training (Karni and Sagi, 1991; Karni et al., 1995; Schoups et al., 1995; Gilbert et al., 2001; Doyon and Benali, 2005; Roelfsema et al., 2010; Sasaki et al., 2010) remain poorly understood. Interestingly, performance increments during skill learning appear larger between consecutive daily training sessions than within sessions (Karni and Sagi, 1993; Schoups et al., 1995; Gervan and Kovacs, 2010), which indicates an offline consolidation process between training sessions. Many studies of the cellular processes contributing to plasticity emphasize the importance of the first few hours after an experience for offline consolidation (Abel and Lattal, 2001; Dudai, 2004), although memory traces likely are subject to consolidation processes over much longer time windows (Lewis, 1979; Nader et al., 2000; Caithness et al., 2004).

In the visual system, areas as low in the anatomical hierarchy as primary visual cortex (V1) can be involved in training-induced neural plasticity and consolidation. Training-induced response changes have been shown after prolonged training (Schoups et al., 2001) but also after a single training session (Schwartz 
et al., 2002; Yotsumoto et al., 2008). Moreover, human neuroimaging (Yotsumoto et al., 2009) has revealed offline V1 activity during sleep $6 \mathrm{~h}$ after a single session of visual skill learning, implicating V1 in offline consolidation after a small amount of training. In contrast, influential theories of visual skill learning (Karni and Bertini, 1997; Ahissar and Hochstein, 2004) predict that the contribution of low-level visual areas to offline consolidation is maximized toward the end of learning (late, asymptotic phase) rather than at the beginning (early phase). The stimulus specificity of perceptual learning during asymptotic but not early learning has been considered a strong argument for the contribution of early visual areas to memory formation in the asymptotic learning phase (Karni and Sagi, 1991; Karni et al., 1995; Schoups et al., 1995; Ahissar and Hochstein, 1997; Gilbert et al., 2001). Here, we investigated, to our knowledge for the first time, whether early indications of plasticity and offline activity in human V1 are functionally relevant for early visual skill learning. We had human participants train on a visual orientation discrimination task and applied repetitive transcranial magnetic stimulation ( $r T M S$ ) to the trained retinotopic location in V1 45 min after the end of the first or second training session. Impaired performance on the day after rTMS would indicate a causally relevant contribution of low-level visual cortex to offline visual skill consolidation during early learning.

\section{Materials and Methods}

\section{Experimental design}

Participants were trained in a visual orientation discrimination task, in which just noticeable differences (JNDs) in orientation were measured for Gabor stimuli presented in the lower-left and upper-right visual quadrant at a fixed peripheral location (Fig. 1A). Training included daily training sessions on 3 consecutive days for both quadrants, presented in blocks and in a counterbalanced order. In one subgroup of participants, training in the lower-left quadrant was followed by training in the upper-right quadrant on the first day ( $L-R)$, and visual quadrant order was alternated on consecutive days (L-R, R-L, L-R: Fig. 1B, Order 1). For the other participants, the learning order was reversed (Fig. 1B, Order 2). rTMS was administered $\sim 45 \mathrm{~min}$ after the end of the first and second daily training session in approximately half of the participants (the other participants served as non-TMS controls). The TMS coil was positioned over right occipital cortex to interfere with processing at the stimulus location in the lower-left visual quadrant ( $\mathrm{L}_{T}$ quadrant) using fMRI-based neuronavigation (Sack et al., 2009). The upper-right visual quadrant was not targeted by TMS ( $\mathrm{R}_{\mathrm{nT}}$ quadrant) and served as a within-subject control quadrant. 
A

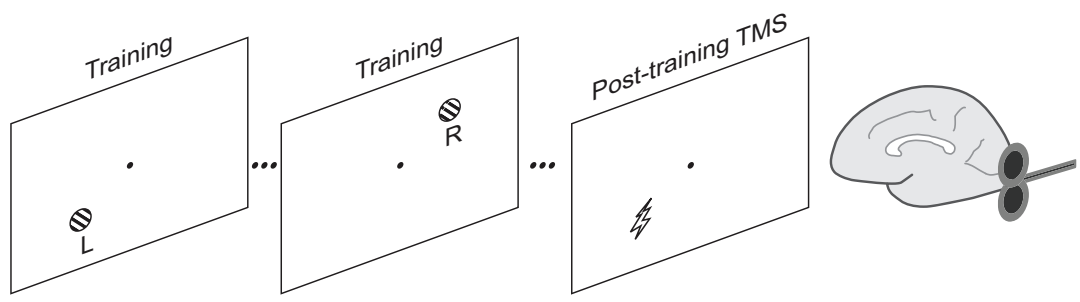

B

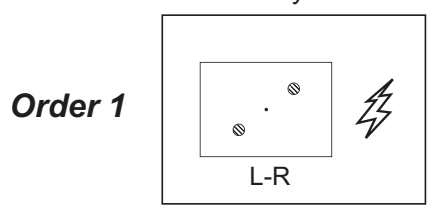

Day 1

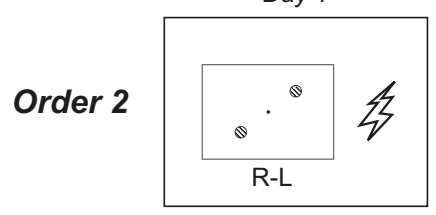

C

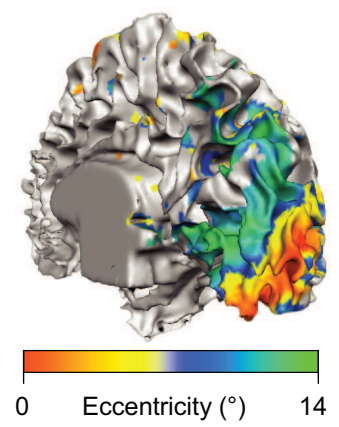

Day 2

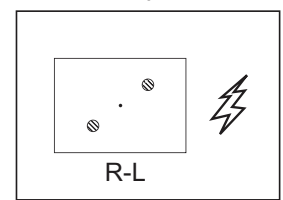

Day 2
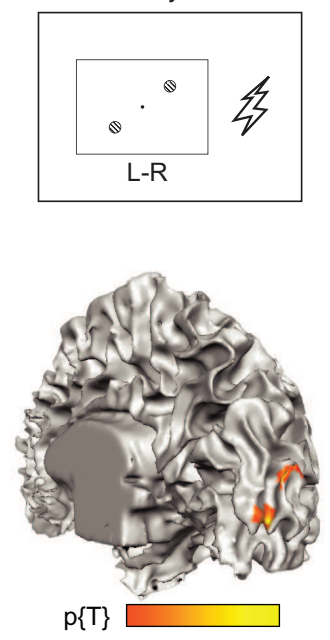

$\mathrm{q}=0.05 \quad \mathrm{p}<1.7 \times \mathrm{e}^{-14}$
Day 3

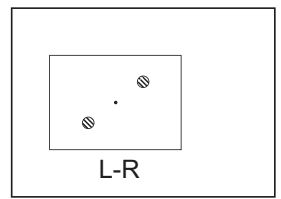

Day 3
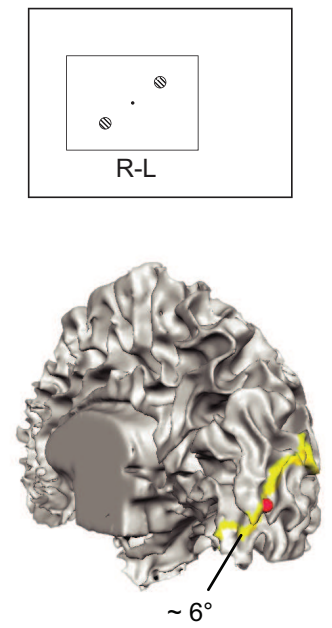

Figure 1. Experimental design. Participants performed an orientation discrimination task on Gabor patches presented either in the lower-left or upper-right visual quadrant (A). Training sessions took place on 3 consecutive days, of which the first two sessions were followed by rTMS in the TMS group (A, B). Training regimens followed one of two possible orders (Order 1 : day $1 \mathrm{~L}-\mathrm{R}$, day $2 \mathrm{R}-\mathrm{L}$, day $3 \mathrm{~L}-\mathrm{R}$; Order 2: $\mathrm{R}-\mathrm{L}, \mathrm{L}-\mathrm{R}$, $\mathrm{R}-\mathrm{L}$ ), related to the quadrant that was trained first on a particular day. After the training session, neuronavigated rTMS was applied to the cortical representation in V1 of the L stimulus (TMS group only) (C). The cortical reconstruction of the right hemisphere of one participant is shown with the superimposed functional results of the eccentricity mapping (left), stimulus localizer (middle), and selected TMS target site (right). In the right image, the approximate localization of $6^{\circ}$ eccentricity (yellow color) according to eccentricity mapping verifies the eccentricity of the target site. The selected TMS target site was constrained to lie within striate cortex (upper bank of the calcarine fissure). 


\section{Participants}

We recruited seven participants for the TMS experiment (three females; mean \pm SD age, $27.4 \pm 2.8$ years) and six participants for the control experiment (two females; mean \pm SD age, $28.6 \pm 4.6$ years). All participants had (corrected to) normal visual acuity, gave written informed consent, and were medically screened for inclusion by an independent physician following published safety guidelines (Rossi et al., 2009). All procedures were approved by the local medical ethics committee of the Maastricht University Medical Centre.

\section{Functional and structural MRI}

TMS target sites on the cortical surface of the right hemisphere were individually determined in each participant of the TMS group using functional MRI localizers. We acquired functional and anatomical images on a 3 tesla Siemens Allegra MR scanner at the Maastricht Brain Imaging Centre at Maastricht University, The Netherlands. Three-dimensional (3D) anatomical T1-weighted images were acquired at an isotropic voxel resolution of $1 \times 1 \times 1 \mathrm{~mm}^{3}$ (192 slices). For functional measurements, we acquired retinotopic maps using polar angle and eccentricity localizers (Sereno et al., 1995; Engel et al., 1997; Linden et al., 1999) (TR/TE, 2000/30 ms; 268 volumes of 28 slices; $128 \times 128$ measurement matrix; $2 \times 2 \mathrm{~mm}^{2}$ in-plane pixel resolution; $2 \mathrm{~mm}$ slice thickness, no gap between slices). We also presented a localizer for the stimulus locations in two separate functional runs, which used the same sequence parameters with the exception of the number of volumes (184 per run).

For the retinotopic localizer runs, we presented a counterclockwise rotating checkerboard wedge (polar mapping) and an expanding checkerboard ring (eccentricity mapping). The luminance of the checkerboard of the stimuli changed every $125 \mathrm{~ms}(8 \mathrm{~Hz})$, and each cycle of rotation or expansion lasted $64 \mathrm{~s}$ (Sereno et al., 1995; Engel et al., 1997; Linden et al., 1999). Each participant saw eight cycle repetitions.

The localizer for perceptual learning location on the visual cortex comprised a checkerboard circle and ring that were presented at the same visual field position as the Gabor patches in the perceptual learning task. The width of the inner dimension of the ring was $3^{\circ}$ (matching the diameter of the circle), and the width of the ring was $1^{\circ}$. The checkerboards of the circle and ring changed color (red-green, green-red, yellow-blue, and blue-yellow) every 125 $\mathrm{ms}(8 \mathrm{~Hz})$ to minimize the occurrence of after-images. The circle and ring were presented in separate blocks in either the lower-left or the upper-right visual field in random order. Brain activity associated with the circle and ring that were presented at the same visual field position was contrasted to optimize the statistical localization of the intended visual field representation in visual cortex (see below). 


\section{Preprocessing and analysis of $\mathrm{FMRI}$ data}

Preprocessing and analysis of the functional and anatomical data were performed using the BrainVoyager QX software version 2.0 (Goebel et al., 2006) (Brain Innovation). The anatomical images were rotated to the anterior-posterior commissure plane (AC-PC plane). Next, the cortical surface of the right hemisphere was segmented and tessellated into a 3D surface representation (Kriegeskorte and Goebel, 2001; Goebel et al., 2006). Also, the outer surface of the head was tessellated into a 3D representation. The 3D representations were used for navigation of the TMS coil over the scalp and cortical surface of each individual participant.

The first two volumes of the functional data were discarded, and the remaining time series were preprocessed using slice scan time correction, headmovement correction, and linear trend removal of the time series and were then rotated to the AC-PC plane and resampled to an isotropic voxel resolution of $3 \times 3 \times 3 \mathrm{~mm}^{3}$. The retinotopy runs (polar and eccentricity mapping) were analyzed using lagged correlation analysis (Linden et al., 1999), with a model of the hemodynamic response to the starting wedge or ring position shifted across 32 lags. Each voxel was color coded according to the model lag of the wedge or ring with the highest correlation.

The localizer for the stimulus position was analyzed using a general linear model (Friston et al., 1995) with four predictors (circle and ring presented in left-lower or right-upper visual field). We defined the functional target location on the upper bank of the calcarine fissure of the right hemisphere by the overlap (logical conjunction) of the statistical contrast between the activity elicited by the right versus left visual field stimulus and the contrast between circle and ring in the left-lower visual field quadrant. We compared the selected target location to the eccentricity mapping results to verify that the target location referred to the presented stimuli at $6^{\circ}$ eccentricity (Fig. 1C). The target location was then marked by a marker on the cortical surface to guide the neuronavigation (see below).

\section{TMS neuronavigation}

For positioning of the TMS coil over the head, we used the BrainVoyager TMS Neuronavigation System (Brain Innovation) (Sack et al., 2009). This system uses ultrasound markers in 3D space to monitor the position of the coil relative to the participant's head. Ultrasound transmitters that continuously transmit pulses to a receiving sensor device are attached to the participant's head and to the TMS coil. Local spatial coordinate systems are created by linking the relative raw spatial position of the ultrasound senders to a set of fixed additional landmarks on the participant's head: the nasion and the two incisurae intertragicae. The specification of these fixed landmarks is achieved via a digitizing pen that also hosts two transmitting ultrasound markers to measure its relative position in $3 \mathrm{D}$ space. The neuronavigation system thus provides topographic 
information of the head-based transmitters relative to a participant-based coordinate frame. The same landmarks digitized on the participant's head are specified on the head reconstruction of the anatomical MR data to achieve TMS-MRI coregistration. After coregistration, events occurring around the head of the participant in real space are registered online and are visualized in real time at correct positions relative to the anatomical reconstruction of the participant's brain. The same system also permits the estimation of the distance between the center of the surface of the coil and the reconstruction of the cortical surface (coil-target distance) and the offset between the target location and the point of entry of the putative magnetic pulse "beam" on the cortical surface (beam-target distance). Through manual adjustment, we aimed to minimize these values during coil positioning and monitored these values for variations during the TMS pulse delivery. Thus, the use of fMRI-localizer-guided neuronavigation maximized the probability that the primary effect of the TMS pulses was in the target location in V1.

\section{TMS}

Primed $1 \mathrm{~Hz}$ rTMS [known to suppress cortical excitability for at least $1 \mathrm{~h}$ (lyer et al., 2003)] was applied to the right visual cortex 45 min after cessation of training on the first and second day, based on the individual fMRI localizer results using neuronavigation techniques (Sack et al., 2009). The biphasic TMS pulses were delivered using a figure-of-eight coil (MCB70) and a MagPro X100 stimulator (Medtronic Functional Diagnostics; maximum stimulator output, 1.9 $\mathrm{T})$. The TMS protocol consisted of a priming phase and a low-frequency $r \mathrm{TMS}$ phase. In the priming phase, 20 trains of 30 pulses were presented at a frequency of $6 \mathrm{~Hz}$ (i.e., each pulse train lasted $5 \mathrm{~s}$ ). Consecutive trains were separated by $25 \mathrm{~s}$. In total, 600 pulses were delivered at an intensity of $45 \%$ maximum stimulator output. The following rTMS phase comprised 400 pulses presented at a frequency of $1 \mathrm{~Hz}$, delivered at $50 \%$ maximum stimulator output.

\section{Perceptual learning task}

Participants performed a visual orientation discrimination task in which they indicated whether a shown stimulus was tilted clockwise or counterclockwise with respect to a (never-shown) reference orientation of $135^{\circ}$ (Orban et al., 1984). Stimuli consisted of Gabor patches ( 0.75 cycles $/{ }^{\circ}$ spatial frequency, $50 \%$ Michelson contrast) that were presented in either the lower-left or upperright visual field $\left(3^{\circ}\right.$ diameter at $6^{\circ}$ eccentricity) on a personal computer monitor (19 inch screen size, $60 \mathrm{~Hz}$ refresh rate, $1280 \times 1024$ pixel resolution) while participants were seated in a dimly lit room. Participants rested their head in a chinrest to stabilize head position relative to the stimulus monitor. A trial started with a blank screen, followed by presentation of a fixation dot at the middle of the screen. In the first $500 \mathrm{~ms}$ of presentation of the fixation dot, 
participants were expected to orient their eyes toward the fixation dot. This was followed by a 750 ms period in which accurate fixation would initiate another $250 \mathrm{~ms}$ period in which fixation was to be maintained, followed by a 500 ms presentation of the Gabor stimulus. Participants responded with their right index or middle finger, respectively, representing a counterclockwise or clockwise deviation from the reference orientation. The response window was 1000 ms. Participants received feedback on their response by brief coloring of the fixation dot (green for correct, red for incorrect). JNDs were determined using a Wetherill and Levitt staircase procedure (Wetherill and Levitt, 1965) tracking $84 \%$ correct, and JNDs were computed as the geometric average of the last 10 reversal points. Stimulus presentation and response recording were performed using Cortex (version 5.9.6; Cortex Software, National Institutes of Health freeware). Furthermore, we used eye-tracking software (Viewpoint EyeTracker version 2.8.3; Arrington Research) with an infrared eye camera (60 Hz sampling rate) to track fixation behavior of the participants. Trials with eye position deviations beyond $1.5^{\circ}$ relative to the fixation dot were aborted and replaced by a new randomly generated trial. Trials in which no response was given were randomly replaced as well.

The protocol consisted of three training sessions scheduled on consecutive days. During each session, five staircases of the orientation discrimination task were performed for each of the two quadrants, tracking $84 \%$ correct performance. The quadrant order was counterbalanced across days/participants, that is, on the first day of learning, half of the participants started with the left-lower quadrant (i.e., completing the order LR-RL-LR across the $3 \mathrm{~d}$ ) and the other half with the right-upper quadrant (completing the order RL-LR-RL across the $3 \mathrm{~d}$ ). Staircases were terminated after 14 reversal points or when a total of 120 trials was reached (on average, $\sim 100$ trials were performed per staircase). During the initial session, starting levels were fixed to $\pm 15^{\circ}$ deviations. On days 2 and 3 , the starting levels were separately determined for both quadrants based on the average performance achieved on the previous day. In total, 30 min of training were spent on each quadrant per day.

\section{Results}

\section{Effect of offline rTMS on learning indices}

Figure 2 shows learning curves and learning indices of representative participants for the two testing orders in both the TMS (A, B) and control (C, D) groups. In each figure panel, testing day and quadrant testing order are shown on top. Line graphs show learning as a function of session for the two quadrants used ( $L_{T}$ quadrant, solid line; $R_{n T}$ quadrant, dashed line), with JNDs normalized within quadrants to the average performance in the first session. In the bar graphs, gray bars ( $R_{n T}$ quadrant) and black bars ( $L_{T}$ quadrant) show learning 
indices quantifying relative improvement between consecutive sessions i - 1 and i by computing the following:

$$
\text { Learning index }=100 \times \frac{\left(J N D_{i-1}-J N D_{i}\right)}{J N D_{i-1}}
$$

A positive index thus indicates learning compared with the preceding session. The indices were computed to quantify learning from the first to second session (left of vertical stippled line) and learning from the second to third session (right from vertical stippled line). All five JNDs contributing to data points per session were used to compute the index, because there was no within-session learning. The absence of within-session learning was confirmed by testing whether, across participants $(n=13)$, the regression coefficients of the lines fitted to the five JNDs in each session were significantly negative (indicating a reduction of threshold size), which was not the case $(\mathrm{t}(38)=-1.7, \mathrm{p}=0.094)$. Positive colored bars (learning index $R_{n T}$ - learning index $L_{T}$ ) show impairment of between-session learning in the $L_{T}$ quadrant.

In Figure 2A, between-session learning after the first session was less strong in the $L_{T}$ quadrant (receiving TMS) compared with the $R_{n T}$ control quadrant, as witnessed by the higher JNDs in $L_{T}$ (filled symbol) compared with the $R_{n T}$ control quadrant (open symbol) in the second session. As a result, learning indices from session 1 to session 2 were higher in the $R_{n T}$ (gray bar) than in the $\mathrm{L}_{\mathrm{T}}$ (black bar) quadrant. However, after the second session, there was strong between-session learning in the $L_{T}$ quadrant because, during the third session, JNDs in the $L_{T}$ quadrant caught up with the level of JNDs in the $R_{n T}$ quadrant (despite the $L_{T}$ quadrant receiving TMS). Consequently, the learning index from session 2 to session 3 was lower in the $R_{n T}$ (gray bar) than in the $L_{T}$ (black bar) quadrant. In Figure 2B, between-session learning after the first session was approximately equally strong in the $L_{T}$ and $R_{n T}$ quadrants, as witnessed by the similar JNDs in the two quadrants during the second session. This resulted in very similar learning indices from session 1 to session 2 in the $R_{n T}$ (gray bar) and $L_{T}$ (black bar) quadrant. However, after the second session, there was no between-session learning in the $L_{T}$ quadrant, as revealed by stagnating JNDs in the $L_{T}$ quadrant during the third session (filled symbol), which were strongly elevated compared with JNDs of the third session in the RnT quadrant (open symbol). As a result, the learning index from session 2 to session 3 was lower in the $L_{T}$ quadrant (black bar) than in the $R_{n T}$ quadrant (gray bar). Hence, the TMS-induced slowing of acquisition in the $L_{T}$ quadrant was present only when TMS was administered after a session with an L-R training order (red bars). When TMS was applied after a session with an R-L training order (blue bars), there was no evidence of slowed acquisition.

Control experiments without TMS in different participants who performed the same training as participants subjected to TMS showed equal acquisition in the two quadrants, regardless of the training order. Figure 2, C and D, shows that JNDs decreased at a comparable rate as a function of session in both 
TMS

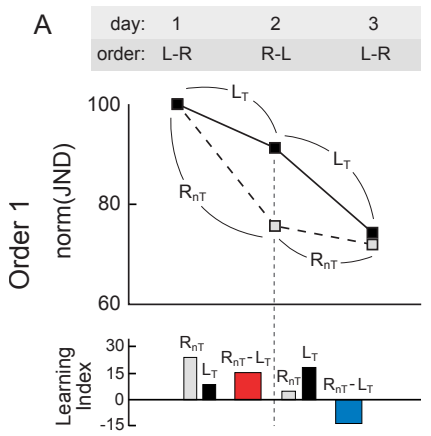

B

$\begin{array}{cccc}\text { day: } & 1 & 2 & 3 \\ \text { order: } & R-L & L-R & R-L\end{array}$
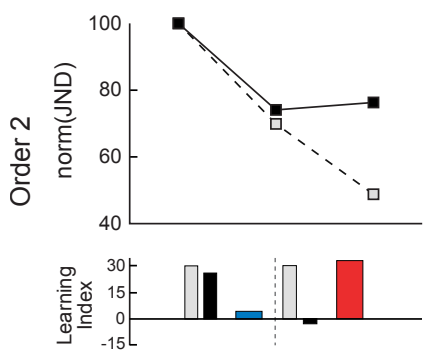

Control

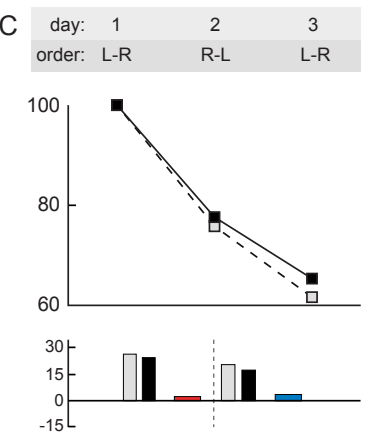

$\begin{array}{rccc}\text { day: } & 1 & 2 & 3 \\ \text { order: } & \text { R-L } & \text { L-R } & \text { R-L }\end{array}$
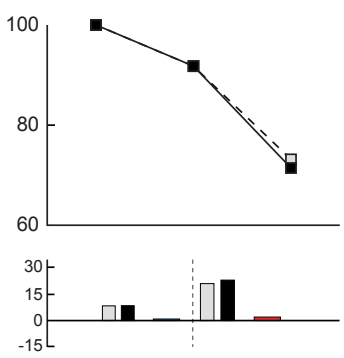

Figure 2. Interaction between TMS interference and learning order. Learning curves for representative TMS (A, B) and control (C, D) participants. Offline TMS hampers learning in the TMS quadrant ( $L_{T}$, solid lines) but not in the control quadrant $\left(R_{n T}\right.$, dashed lines) (A, B). Moreover, the time point of interference depends on the learning order: for the order 1 training scheme $(L-R, R-L, L-R)$, the TMS-induced interference is clearly visible in session $2(A)$; for the order 2 training scheme ( $R-L, L-R, R-L)$, interference only occurs in session 3 (B). The bar graphs in each of the panels show learning indices that quantify the relative improvement between consecutive sessions separately for both quadrants $\left(R_{n T}\right.$, gray bars; $L_{T}$, black bars). Colored bars represent the differences in learning between the $R_{n T}$ and $L_{T}$ quadrant (after $L-R$ training, red bars; after R-L training, blue bars). Conjointly, these findings indicate that the TMS intervention leads to spatially specific learning impairments that exclusively emerge when the targeted quadrant is trained first (i.e., after L-R sessions). Conversely, no differences were found between the left and right quadrant in either training order in the control participants (C, D). We propose that ending a training session with the $R_{n T}$ quadrant leads to an uncoupling of retinotopic regions representing the trained $L_{T}$ quadrant from high-level areas that help guide the plastic changes in these retinotopic regions leading to memory formation. This uncoupling may leave low-level areas representing the $L_{T}$ quadrant vulnerable to offline TMS interference (see Fig. 4). Average SEMs of normalized JNDs were 4.27, 6.26, 3.25, and 4.20, respectively, for plots A-D (not shown for clarity). 


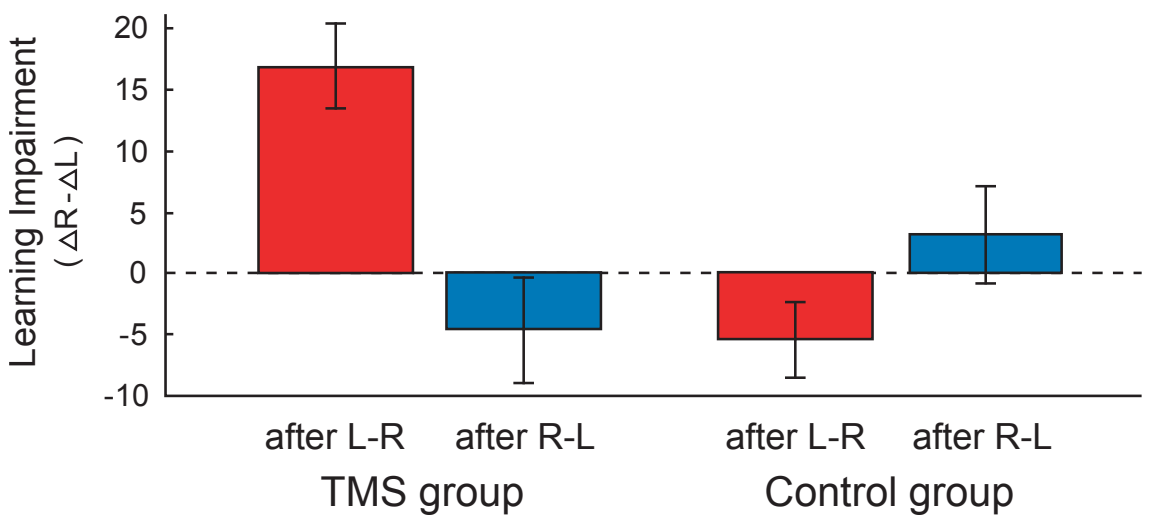

Figure 3. Group-level TMS-induced learning impairment specific for $L-R$ learning order. The bar graph shows a quantification of the TMS-induced learning impairment (defined as the difference between learning indices for the $L_{T}$ and $R_{n T}$ quadrant) when pooling the data across participants (TMS, $n=7$; control, $n=6$ ) according to the followed learning order (i.e., corresponding to a pooling of the red vs blue color-coded bars in Fig. 2). In the TMS group, learning is clearly impaired after L-R training (red bar), whereas TMS has no effect after R-L training (blue bar). In the control group, learning is very similar in both quadrants regardless of the followed training order. For interpretation, see Figure 4 and Discussion.

quadrants tested, resulting in similar learning indices in each quadrant (gray and black bars). The order of training did not appear to influence the learning rate in the two quadrants.

To test the effect of training order in the two groups statistically, the $R_{n T}$ $L_{T}$ difference scores between learning indices were pooled over all participants in the TMS group $(\mathrm{n}=7$ ) within $\mathrm{L}-\mathrm{R}$ order (Fig. 2, red bars) and within $\mathrm{R}-\mathrm{L}$ order (Fig. 2, blue bars), and the same was done within the control group $(n=6)$ (Fig. 3). The difference scores quantify the learning impairment induced by TMS, with positive values indicating impairment. We then applied a mixed-effects repeated-measures ANOVA to the learning impairment indices (Fig. 3). As a between-subject factor, we used group (TMS, control), and as a within-subject factor, we used order (order 1 , order 2 ). The analysis yielded a significant interaction effect of group $x$ order $(F(1,11)=27.7, p<0.001)$. This interaction was attributable to a significant reduction of learning in the $L_{T}$ quadrant for rTMS after $L-R$ sessions (red bar; $t(6)=5.0, p=0.002$, one-sample $t$ test), which was absent in the $L_{T}$ quadrant after $R-L$ sessions (blue bar; $\mathrm{t}(6)=-1.0, \mathrm{p}=0.35$ ) and also absent in the $\mathrm{L}_{\mathrm{nT}}$ quadrant of the control group for both training orders (after $\mathrm{L}-\mathrm{R}, \mathrm{t}(5)=-1.9, \mathrm{p}=0.11$; after $\mathrm{R}-\mathrm{L}, \mathrm{t}(5)=0.8, \mathrm{p}=0.49)$.

The differential effect of $r T M S$ after $R-L$ and $L-R$ training orders in the TMS group was not attributable to a difference between training orders in the 
time elapsed from end of training to onset of rTMS in the $L_{T}$ quadrant. The presence of some variation in the precise timing of TMS among sessions and subjects allowed us to compute a correlation between these time intervals and the difference in learning impairments between training orders. This correlation was not significant $(r=-0.04, p>0.93)$, indicating that the different findings for the two training orders truly represent an order effect.

\section{Verification of results using JNDs and learning indices}

In the following two analyses, we verified that the previous findings were not an artifact of the compression of the data into the learning impairment index used in Figure 3. Therefore, in one analysis, we entered the log-transformed JNDs of each session of the TMS and control participants into a mixed-model repeated-measures ANOVA with group (TMS, control) and order (order 1, order 2 ) as between-subject factors and session (1, 2, or 3) and quadrant $\left(L_{T}, R_{n T}\right)$ as within-subject factors. Results showed a significant main effect of session $(F(2,18)=237.0, p<0.001)$ and significant interaction effects of session $x$ order $(F(2,18)=4.4, p=0.029)$, session $\times$ Group $(F(2,18)=6.7, p=0.007)$, session $\times$ group $\times$ order $(F(2,18)=5.5, p=0.014)$ and session $\times$ group $\times$ order $x$ quadrant $(F(2,18)=4.4, p=0.028)$. The main effect of session indicated that participants improved on their performance across the three sessions. The interaction effects indicated that participants of the TMS group, but not the control group, showed differential improvements between the two quadrants, which occurred at different moments in the learning curve depending on training order.

In another analysis, we performed a repeated-measures ANOVA of the learning indices, using as between-subject factors group and order and as withinsubject factors learning period (after session 1, after session 2) and quadrant. The data that were entered in this analysis correspond to the gray and black bars in the bar graphs in Figure 2, i.e., before computing the learning impairment index $R_{n T}-L_{T}$. Results revealed significant interaction effects of learning period $\times$ group $(F(1,9)=21.0, p=0.001)$, learning period $\times$ group $\times$ order $(F(1,9)=31.8, p<0.001)$ and learning period $\times$ group $\times$ order $\times$ quadrant $(F(1,9)=24.9, p=0.001)$. Thus, these interaction effects corroborate the repeated-measures ANOVA of the JNDs and thereby the effects reported in Figure 3.

\section{Verification of between-session consolidation (offline learning)}

In our main analyses, we quantified offline learning indices as the normalized difference between the average JNDs of consecutive learning sessions, thereby assuming that there was no improvement during training. This assumption is defensible because statistical analysis showed that the trend for within-session 
improvement was not significant. Conversely, a small trend seemed present, and therefore we reanalyzed the data estimating between-session improvement as the difference between the average of the last two JNDs of training session $\mathrm{i}-1$ and the average of the first two JNDs of session $\mathrm{i}$ :

$$
\text { index }=\frac{\left(J N D_{i-1,4}+J N D_{i-1,5}\right)}{2}-\frac{\left(J N D_{i, 1}+J N D_{i, 2}\right)}{2}
$$

Here, higher positive values indicated more between-session improvement. We arranged these values according to training order and entered them into a repeated-measures ANOVA with factors quadrant (left, right), learning period (after session 1, after session 2), and group (TMS, control). Only the interaction term quadrant $x$ learning period $x$ group was significant $(F(1,11)=10.1$, $p=0.009)$, which replicates the core finding shown in Figure 3.

\section{Discussion}

Our study presents three new findings. First, we present the first causal evidence for a behaviorally relevant contribution of low-level visual areas to offline consolidation in a visual skill learning task. Second, this contribution occurred surprisingly early in the time course of learning. Third, the contribution of early visual cortex depended on modulations in the offline brain state induced by the different training orders.

In light of two influential theories of perceptual learning, the early contribution of low-level sensory areas in skill consolidation may be qualified as unexpected. According to the "lowest-level " theory (Karni and Bertini, 1997), consolidation processes induced by training on a specific stimulus parameter take place in the most low-level cortical areas that most efficiently encode that parameter. Here, we used an orientation discrimination task, and therefore consolidation is expected in low-level visual areas in which neurons show strong orientation tuning. Furthermore, lowest-level theory postulates that the greatest changes will occur late in learning when task demands exceed sensory limits. In a previous neurophysiological study in monkeys (Schoups et al., 2001), changes in orientation tuning were recorded in V1 neurons stimulated passively (stimuli ignored by the monkeys). These tuning changes were observed after lengthy orientation discrimination training and likely represent a memory trace that resulted from offline consolidation after training sessions in the asymptotic learning phase. We suggest that the finding of interference caused by rTMS during the offline period after training sessions early in learning is unlikely to reflect interference with the plastic processes that lead to modification of neuronal tuning properties in V1 or other low-level visual areas. This is because, in our experiment, no lengthy training has taken place that would have tested the limits of sensory encoding at the lowest levels in the visual system. Moreover, even if there had 
been a change in the automatic filtering properties of $\mathrm{V} 1$ neurons (as measured with ignored stimuli), this would not explain the conditional nature of the rTMS-induced interference.

According to reverse hierarchy theory (Ahissar and Hochstein, 2004), performance enhancements depend on higher-level areas establishing better access to increasingly more specific information at progressively lower levels in the visual system. Hence, the plasticity required to embed readout routines at increasingly lower levels in the visual system (consolidation) would occur toward the end of learning rather than at the beginning. Our data, in contrast, indicate that low-level visual areas are already accessed at the very beginning of learning. Therefore, there may not only be a late recruitment of low-level visual areas reflecting a specificity-driven process designed to gain access to detailed sensory information but also an early recruitment reflecting the setting up of synaptic connectivity in low-level areas for readout routines with the purpose of later fine-tuning ( $\mathrm{Li}$ et al., 2004). We suggest that, because of this, large neural networks that include low- to high-level areas may play a role in offline consolidation from the beginning of learning (Lewis et al., 2009). Reports of changes in stimulus-driven activation observed with fMRI early in visual skill learning (Schwartz et al., 2002; Mukai et al., 2007; Yotsumoto et al., 2008), of reverberating activity in large functional networks after a learning experience (Hoffman and McNaughton, 2002; Ji and Wilson, 2007; Tambini et al., 2010), and of behavioral interference early in visual learning (Seitz et al., 2005) all support this possibility. Interfering input, such as delivered by TMS, may reset the synaptic weights of readout routines more easily when the network that maintains them is smaller (Buonomano and Maass, 2009) and does not include high-level areas that during offline consolidation may help strengthening the synaptic weights appropriate for the task (Fig. 4). In this view, training in the $L_{T}$ quadrant followed by $R_{n} T$ training before TMS leads to a brain state in which low-level areas representing the lower-left quadrant are uncoupled from high-level areas, thereby increasing the vulnerability of plastic processes in the low-level areas to TMS interference (Fig. 4, top row). Conversely, training in the $L_{T}$ quadrant after $R_{n T}$ training leads to a brain state in which low-level networks representing the lower-left quadrant remain coupled with high-level networks while TMS is delivered, thereby decreasing the vulnerability of processes in the low-level areas to TMS interference (Fig. 4, bottom row). We suggest this is why TMS directed to the lower-left quadrant interfered with consolidation and learning after an $\mathrm{L}-\mathrm{R}$, but not after an $\mathrm{R}-\mathrm{L}$, training order. Remarkably, the contribution of low-level visual areas to offline consolidation depended on contextual aspects in a manner unexpected for areas low in the visual hierarchy but in agreement with characteristics of early skill learning (Karni and Bertini, 1997; Ahissar and Hochstein, 2004).

We have interpreted our data in light of a coupling between high- and lowlevel networks that protects a memory trace in low-level areas from TMS interference, and it is likely that attention is a factor that contributes to the 
A

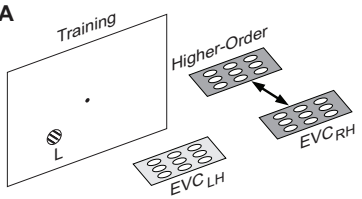

B
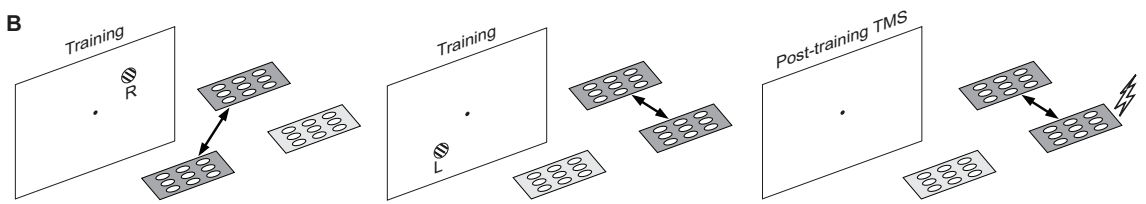

Figure 4. Graphical depiction of the proposed mechanism underlying the interaction between TMS and learning order. A, L-R learning order. In the first block (left), coupling between higher-order areas and early visual cortex (EVC) is stronger (represented by dark shading and arrow) in the right hemisphere (as a result of active processing of visual stimuli presented in the left hemifield). This more intense coupling then switches to the left hemisphere during the second block (right visual field stimulation; middle). Finally, the induced interareal coupling persists during the offline consolidation phase and is stronger in the hemisphere that was most recently involved in active task performance (right). Consequently, TMS to the (now uncoupled) right hemisphere is effective in disrupting ongoing consolidation processes. $B$, The same chain of events but now for the R-L training order. Here, TMS is not effective as the targeted visual cortex is still coupled to the higher-order areas in the functional network. 
instatement of this coupling. Several studies indicate that repeated exposure to stimuli that are not used in a task, or even presented outside visual awareness, can also produce memory traces (Watanabe et al., 2001, 2002; Gutnisky et al., 2009; for review, see Seitz and Watanabe, 2009). Mere exposure to stimuli produces bottom-up activity leading to memory traces that are likely limited to low-level sensory areas (Watanabe et al., 2002). Thus, if coupling between high- and low-level areas is the crucial factor protecting memory traces in lowlevel areas from TMS interference, then a large contribution of mere stimulus exposure to the consolidation process studied here is unlikely.

Recently, Cohen and Robertson (2011) reported that offline rTMS after a pair of tasks prevented retroactive interference by the second task on the first task. Specifically, when word list learning was followed by a motor task, subsequent offline TMS over dorsolateral prefrontal cortex decreased interference of the motor task onto the word task. Conversely, when motor learning was followed by word list learning, subsequent offline TMS over motor cortex decreased interference of the word list task onto the motor task. The reduced interference after TMS appears to contradict the increased interference after TMS reported in our study. However, in a control condition, Cohen and Robertson (2011) did report interference when TMS followed performance in a single task, in line with our own study. In our study, performance in a single type of task executed at one location did not interfere with performance when the same task was executed in another location (Fig. 2C,D), but under specific conditions TMS could interfere with consolidation of the task performed in one of the locations. Therefore, any TMS effect we reported after a single task cannot be related easily to the retroactive interference between different tasks and its reduction by TMS, as reported by Cohen and Robertson (2011). Nevertheless, both our own study and that of Cohen and Robertson (2011) show a state-dependent effect of TMS, which may differ between studies because of the nature of the network interactions that contribute to consolidation. Cohen and Robertson's (2011) data suggest an antagonistic form of interactions between two networks contributing to two tasks, which when abolished by TMS, restores consolidation and subsequent performance. In our study, we postulated a cooperative interaction between higher and lower levels of the sensory system, which when abolished or weakened by task conditions makes consolidation processes at lower levels more vulnerable to TMS interference.

Although TMS was directed to $\mathrm{V} 1$, the induced change in activity may have extended to other areas. Therefore, it could be argued that our results were attributable to remote TMS effects in distant network nodes that in turn would have secondarily affected early visual areas. If true, this would invalidate the interpretation of our data as revealing a behaviorally relevant contribution of low-level visual areas to skill consolidation in the earliest phases of learning. Remote activation changes via TMS have been described in several domains (Bestmann et al., 2004; Ruff et al., 2006; Sack et al., 2007; for review, see Reithler et al., 2011) and could have extended from V1 into V2 or V3. Nev- 
ertheless, significant behaviorally relevant remote effects of TMS to date have only been described in the top-down direction by direct stimulation of higherorder areas (Ruff et al., 2006). So far, there is no evidence that significant and behaviorally relevant top-down effects in higher-order areas can be induced indirectly by bottom-up effects caused by TMS stimulation of V1. More importantly, remote TMS-induced effects are state dependent in the sense that local increases in activation and interareal functional coupling induced by TMS are stronger when the targeted site belongs to an active network (Morishima et al., 2009; Blankenburg et al., 2010). Consequently, in our study, indirect remote effects in high-level areas induced by TMS to V1 should be strongest when low- and high-level areas are coupled. This was likely the case when the most recently trained retinotopic locus was targeted by TMS. Therefore, a learning impairment ascribed to remote TMS effects should be highest when the session ended with training in $L_{T}$ compared with when the session ended with training in $R_{n T}$, yet we found the opposite.

In summary, our findings indicate that training-induced plasticity during early learning of visual discriminations can take place simultaneously in a large network incorporating the lowest levels of the visual hierarchy, including V1. Offline consolidation in early visual areas occurs early in the time course of learning and is behaviorally relevant, and its robustness is modulated by task context. These findings reveal a new and unexpected role of early visual cortex in visual skill learning.

\section{References}

Abel T, Lattal KM (2001) Molecular mechanisms of memory acquisition, consolidation and retrieval. Curr Opin Neurobiol 11:180-187.

Ahissar M, Hochstein S (1997) Task difficulty and the specificity of perceptual learning. Nature 387:401-406.

Ahissar M, Hochstein S (2004) The reverse hierarchy theory of visual perceptual learning. Trends Cogn Sci 8:457-464.

Bestmann S, Baudewig J, Siebner HR, Rothwell JC, Frahm J (2004) Functional MRI of the immediate impact of transcranial magnetic stimulation on cortical and subcortical motor circuits. Eur J Neurosci 19:1950-1962.

Blankenburg F, Ruff CC, Bestmann S, Bjoertomt O, Josephs O, Deichmann R, Driver J (2010) Studying the role of human parietal cortex in visuospatial attention with concurrent TMS-fMRI. Cereb Cortex 20:2702-2711.

Buonomano DV, Maass W (2009) State-dependent computations: spatiotemporal processing in cortical networks. Nat Rev Neurosci 10:113-125.

Caithness G, Osu R, Bays P, Chase H, Klassen J, Kawato M, Wolpert DM, Flanagan JR (2004) Failure to consolidate the consolidation theory of learning for sensorimotor adaptation tasks. J Neurosci 24:8662-8671.

Cohen DA, Robertson EM (2011) Preventing interference between different memory tasks. Nat Neurosci 14:953-955. 
Doyon J, Benali H (2005) Reorganization and plasticity in the adult brain during learning of motor skills. Curr Opin Neurobiol 15:161-167.

Dudai Y (2004) The neurobiology of consolidations, or, how stable is the engram? Annu Rev Psychol 55:51-86.

Engel SA, Glover GH, Wandell BA (1997) Retinotopic organization in human visual cortex and the spatial precision of functional MRI. Cereb Cortex 7:181-192.

Friston KJ, Holmes AP, Poline JB, Grasby PJ, Williams SC, Frackowiak RS, Turner R (1995) Analysis of fMRI time-series revisited. Neuroimage 2:45-53.

Gervan P, Kovacs I (2010) Two phases of offline learning in contour integration. J Vis 10:24. Gilbert CD, Sigman M, Crist RE (2001) The neural basis of perceptual learning. Neuron 31:681-697.

Goebel R, Esposito F, Formisano E (2006) Analysis of functional image analysis contest (FIAC) data with Brainvoyager QX: from single-subject to cortically aligned group general linear model analysis and self- organizing group independent component analysis. Hum Brain Mapp 27:392-401.

Gutnisky DA, Hansen BJ, Iliescu BF, Dragoi V (2009) Attention alters visual plasticity during exposure-based learning. Curr Biol 19:555-560.

Hoffman KL, McNaughton BL (2002) Sleep on it: cortical reorganization after-the-fact. Trends Neurosci 25:1-2.

lyer MB, Schleper N, Wassermann EM (2003) Priming stimulation enhances the depressant effect of low-frequency repetitive transcranial magnetic stimulation. J Neurosci 23:10867-10872.

Ji D, Wilson MA (2007) Coordinated memory replay in the visual cortex and hippocampus during sleep. Nat Neurosci 10:100-107.

Karni A, Bertini G (1997) Learning perceptual skills: behavioral probes into adult cortical plasticity. Curr Opin Neurobiol 7:530-535.

Karni A, Sagi D (1991) Where practice makes perfect in texture discrimination: evidence for primary visual cortex plasticity. Proc Natl Acad Sci USA 88:4966-4970.

Karni A, Sagi D (1993) The time course of learning a visual skill. Nature 365:250-252.

Karni A, Meyer G, Jezzard P, Adams MM, Turner R, Ungerleider LG (1995) Functional MRI evidence for adult motor cortex plasticity during motor skill learning. Nature 377:155-158. Kriegeskorte N, Goebel R (2001) An efficient algorithm for topologically correct segmentation of the cortical sheet in anatomical mr volumes. Neuroimage 14:329-346. Lewis CM, Baldassarre A, Committeri G, Romani GL, Corbetta M (2009) Learning sculpts the spontaneous activity of the resting human brain. Proc Natl Acad Sci USA 106:17558-17563.

Lewis DJ (1979) Psychobiology of active and inactive memory. Psychol Bull 86:1054-1083. Li W, Pieĺch V, Gilbert CD (2004) Perceptual learning and top-down influences in primary visual cortex. Nat Neurosci 7:651-657.

Linden DE, Kallenbach U, Heinecke A, Singer W, Goebel R (1999) The myth of upright vision. A psychophysical and functional imaging study of adaptation to inverting spectacles. Perception 28:469-481.

Morishima Y, Akaishi R, Yamada Y, Okuda J, Toma K, Sakai K (2009) Task- specific signal transmission from prefrontal cortex in visual selective attention. Nat Neurosci 12:85-91. 
Mukai I, Kim D, Fukunaga M, Japee S, Marrett S, Ungerleider LG (2007) Activations in visual and attention-related areas predict and correlate with the degree of perceptual learning. J Neurosci 27:11401-11411.

Nader K, Schafe GE, LeDoux JE (2000) The labile nature of consolidation theory. Nat Rev Neurosci 1:216-219.

Orban GA, Vandenbussche E, Vogels R (1984) Human orientation discrimination tested with long stimuli. Vision Res 24:121-128.

Reithler J, Peters JC, Sack AT (2011) Multimodal transcranial magnetic stimulation: using concurrent neuroimaging to reveal the neural network dynamics of noninvasive brain stimulation. Prog Neurobiol 94:149-165.

Roelfsema PR, van Ooyen A, Watanabe T (2010) Perceptual learning rules based on reinforcers and attention. Trends Cogn Sci 14:64-71.

Rossi S, Hallett M, Rossini PM, Pascual-Leone A; Safety of TMS Consensus Group (2009)

Safety, ethical considerations, and application guidelines for the use of transcranial magnetic stimulation in clinical practice and research. Clin Neurophysiol 120:2008-2039.

Ruff CC, Blankenburg F, Bjoertomt O, Bestmann S, Freeman E, Haynes JD, Rees G, Josephs O, Deichmann R, Driver J (2006) Concurrent TMSfMRI and psychophysics reveal frontal influences on human retinotopic visual cortex. Curr Biol 16:1479-1488.

Sack AT, Kohler A, Bestmann S, Linden DE, Dechent P, Goebel R, Baudewig J (2007) Imaging the brain activity changes underlying impaired visuospatial judgments: simultaneous FMRI, TMS, and behavioral studies. Cereb Cortex 17:2841-2852.

Sack AT, Cohen Kadosh R, Schuhmann T, Moerel M, Walsh V, Goebel R (2009) Optimizing functional accuracy of TMS in cognitive studies: a comparison of methods. J Cogn Neurosci 21:207-221.

Sasaki Y, Nanez JE, Watanabe T (2010) Advances in visual perceptual learning and plasticity. Nat Rev Neurosci 11:53-60.

Schoups AA, Vogels R, Orban GA (1995) Human perceptual learning in identifying the oblique orientation: retinotopy, orientation specificity and monocularity. J Physiol 483:797-810.

Schoups A, Vogels R, Qian N, Orban G (2001) Practising orientation identification improves orientation coding in V1 neurons. Nature 412:549 -553.

Schwartz S, Maquet P, Frith C (2002) Neural correlates of perceptual learning: a functional MRI study of visual texture discrimination. Proc Natl Acad Sci USA 99:17137-17142.

Seitz AR, Watanabe T (2009) The phenomenon of task-irrelevant perceptual learning. Vision Res 49:2604-2610.

Seitz AR, Yamagishi N, Werner B, Goda N, Kawato M, Watanabe T (2005) Task-specific disruption of perceptual learning. Proc Natl Acad Sci USA 102:14895-14900.

Sereno MI, Dale AM, Reppas JB, Kwong KK, Belliveau JW, Brady TJ, Rosen BR, Tootell RB (1995) Borders of multiple visual areas in humans revealed by functional magnetic resonance imaging. Science 268:889-893.

Tambini A, Ketz N, Davachi L (2010) Enhanced brain correlations during rest are related to memory for recent experiences. Neuron 65:280-290.

WatanabeT,Náñez JE,SasakiY (2001) Perceptuallearningwithoutperception. Nature 413:844-848. 
Watanabe T, Náñez JE Sr, Koyama S, Mukai I, Liederman J, Sasaki Y (2002) Greater plasticity in lower-level than higher-level visual motion processing in a passive perceptual learning task. Nat Neurosci 5:1003-1009.

Wetherill GB, Levitt H (1965) Sequential estimation of points on a psychometric function. Br J Math Stat Psychol 18:1-10.

Yotsumoto Y, Watanabe T, Sasaki Y (2008) Different dynamics of performance and brain activation in the time course of perceptual learning. Neuron 57:827-833.

Yotsumoto Y, Sasaki Y, Chan P, Vasios CE, Bonmassar G, Ito N, Nat́n ezJESr, Shimojo S, Watanabe $T$ (2009) Location-specific cortical activation changes during sleep after training for perceptual learning. Curr Biol 19:1278-1282. 


\section{Lasting memory traces of visual skill learning in human V1}

based on:Been, M., Valente, G., Mussgens, D., Jans, B., De Weerd, P., Lasting memory traces of visual skill learning in human V1. In Preparation 


\section{Abstract}

Controversial new views hold that primary sensory cortex does not contribute to long-term storage of expertise related to perceptual skills. However, nine weeks after completing visual orientation discrimination training, we found that voxelwise patterns of fMRI activity in human striate (but not extrastriate) cortex carried increased information about stimulus orientation in the previously trained stimulus conditions. This shows V1's behavioural relevance for the long-term representation of visual expertise.

\section{Introduction}

According to classical views of perceptual learning, prolonged training in a perceptual task leads to asymptotic, stimulus-specific enhancements in performance that are due to plastic changes in low-level sensory areas (Karni, 1996; Fahle, 2005). This view has been supported by neurophysiological studies reporting tuning changes in primary sensory neurons after prolonged training (Recanzone et al., 1993; Schoups et al., 2001) as well as topographic remapping (Kaas et al., 1990; Recanzone et al., 1992). However, the existence of behaviourally relevant plasticity in primary sensory areas has been challenged by several new lines of evidence. First, psychophysical studies have questioned the stimulus specificity of perceptual learning, thereby putting the contribution of early sensory areas to the formation of skill memory in doubt (Xiao et al., 2008). Second, neurophysiological studies (Law and Gold, 2008) and fMRI studies (Zhang and Kourtzi, 2010) have demonstrated perceptual learning without measurable correlates of plasticity in visual primary cortex (V1) at the conclusion of training. Third, a carefully performed combined electrophysiologi$\mathrm{cal} / \mathrm{fMRI}$ study failed to confirm topographic remapping in V1 following retinal lesions, which poses a serious challenge to earlier studies reporting this phenomenon (Smirnakis et al., 2005). Hence, there is accumulating evidence that provides little support for the idea of significant plasticity in early sensory areas contributing to long-lasting memory traces related to perceptual skills. Instead, new proposals are emerging in which primary sensory cortex may contribute to the acquisition of perceptual skills, but not to the formation of long-term memory traces (Yotsumoto et al., 2008; Reed et al., 2011).

Here, we used $\mathrm{fMRI}$ to test the existence of long-term memory traces in primary visual cortex (V1) in a visual perceptual learning paradigm. Because some studies might have failed to detect memory-related plasticity due to their choice of stimuli (Zhang and Kourtzi, 2010), or insufficient training (Xiao et al., 2008), we used square wave gratings, known to drive V1 neurons well, and trained participants $(\mathrm{N}=6)$ in an orientation discrimination task at a fixed orientation difference, in which grating contrast was adapted to performance, until they reached asymptotic performance (average length of training 11.3 daily sessions, see Methods). Nine weeks after termination of training, we used 
Multi Voxel Pattern Analysis (MVPA) of fMRI data (Norman et al., 2006; Mur et al., 2009) to test whether distributed activation patterns in 'trained' regions of V1 contain more information to classify grating orientation than response patterns in 'untrained' regions.

\section{Materials en Methods}

\section{Participants}

Seven healthy participants (1 male, six females, age 22-35 years) had normal or corrected to normal visual acuity, gave written informed consent for all parts of the experiment, were financially compensated, and were debriefed following completion of data acquisition (same participants as in chapter 5). One subject was removed from data analyses, because of excessive head motion during the fMRI MVPA scan session. All aspects of the experiments were in accordance with formal approval by the Psychology and Neuroscience Faculty Ethical Committee at Maastricht University. All participants were experienced psychophysical participants who had no experience with the specific tasks used in the present experiment.

\section{Experimental Design}

As the time course of contrast-training in an orientation discrimination task at constant orientation difference was unknown, learning curves were first determined in the psychophysics lab for one of the two lower quadrants of the visual field (Figure 1A, lower quadrant picked at random in different participants). The training started by estimating personal orientation thresholds at a high contrast $(16 \%)$ in a single session of orientation training ( 8 thresholds estimating $84 \%$ correct with a Wetherill and Levitt staircase procedure, 1965). Across participants, this resulted in average thresholds of 7.2 degrees. This value was then divided by 2 to estimate the hypothetical performance on the next day, resulting in an average orientation difference of 3.6 degrees. At that orientation difference, at reference orientation $135^{\circ}$, training was continued by making contrast dependent on performance (84\% correct staircase procedure, Wetherill and Levitt, 1965). Average asymptotic performance converged on a contrast threshold of $4.25 \%$, and the total average length of the initial learning curve was 6 sessions.

Pilot experiments showed that this performance was not maintained in the scanner, and we found that equivalent performance in the scanner at contrasts used in the psychophysics lab was only possible after doubling the orientation difference at which participants had performed their training in the psychophysics lab. (This may be due to the slightly blurred image produced by the back-projection system used in the scanner.) The training used for the purpose of $\mathrm{FMRI}$ measurements was designed based on that finding. Thus, training 
started again in a new quadrant (an upper quadrant, Figure 1B), in the same participants who had participated in the initial training in the lower quadrant of the same hemifield (Figure $1 \mathrm{~A}$ ). We used the same fixed orientation difference as determined at the outset of the first training $\left(3.6^{\circ}\right)$, doubled $\left(7.2^{\circ}\right)$ for measurements in the scanner (Figure 1C, D).

The main goal of the study was to compare MVPA in a trained (upper quadrant, Figure 1E) and a control quadrant (diagonally opposite in the other hemifield) after the conclusion of training. The trained upper quadrant was chosen to be above the lower quadrant in which initial training had taken place (Figure 1A), so that the diagonally opposite control stimuli would not coincide with the location of initial training. The complete course of behavioural training took place over an average of 11.3 sessions, with on average 6.7 sessions taking place in the psychophysics lab, and 4.6 sessions in the fMRI scanner (at doubled orientation difference).

The constant stimuli method (involving data collection at a set of 8 fixed contrasts ranging from $1 \%$ to $32 \%$ ) was used for the collection of longitudinal fMRI learning data, which fall outside the scope of the present report. However, In the present report, both the behavioural data obtained in the scanner and those obtained in the psychophysics lab will be used to illustrate the extent of skill learning from beginning to end of training. The learning progress measured in the psychophysics lab with staircase thresholds may have profited from the additional experience in the scanner.

To match the control quadrant with the training quadrant, each stimulus in the trained quadrant was paired with an ignored stimulus of matched contrast in the diagonally opposite Control quadrant, chosen randomly from the two orientation alternatives used in the trained quadrant. The presentation of matching stimuli was performed both during staircase measurements in the psychophysics lab and during constant stimulus measurements in the scanner.

Stimuli used in the MVPA design were presented in a similar fashion as during the behavioural training sessions (in the psychophysics lab and the scanner). However stimuli were presented outside the focus of attention, while participants performed an attentional demanding fixation task (Figure 1E, F) (Kastner et al., 1998).

\section{Stimuli, stimulus delivery and responses in psychophysics lab}

Stimuli consisted of square wave gratings (2c/deg spatial frequency, presented on a PC monitor (19 inch TFT screen, $60 \mathrm{~Hz}$ refresh rate, $1280 \times 1024$ pixel resolution, $5^{\circ}$ diameter, $5^{\circ}$ eccentricity, phase-randomized). Participants were seated in a dimly-lit room. Average luminance of stimuli and of the grey background was $56 \mathrm{~cd} / \mathrm{m}^{2}$. Participants placed their head in a chin rest to stabilize head position relative to the stimulus monitor. Eye position was monitored with an infrared eye camera (60 Hz sampling rate Viewpoint Eye Tracker v.2.8.3, 

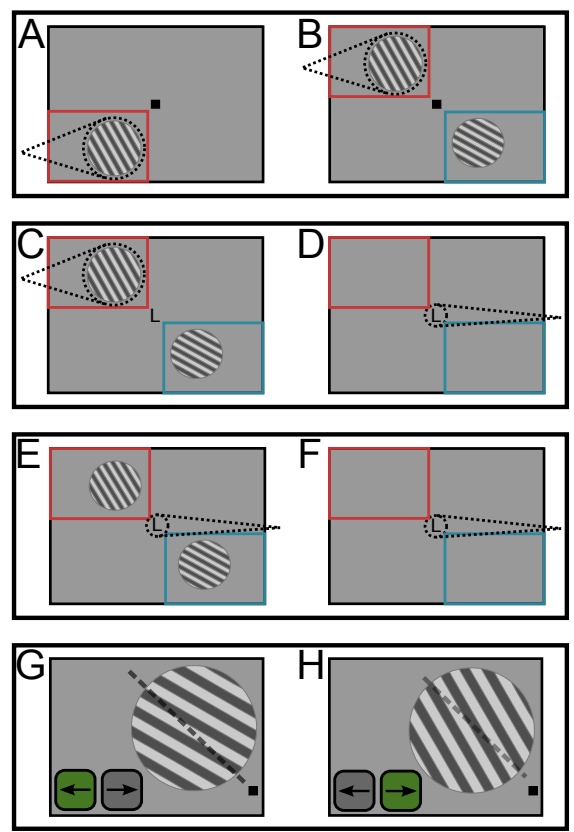

Figure 1. Experimental design. Participants fixated the screen's centre, covertly attended and discriminated the stimulus in one of the visual field quadrants, depending on the experimental condition (Trained quadrant, red frame), and ignored the stimulus in the diametrically opposite quadrant (Control quadrant, blue frame). Cone shows allocation of attention. (A) Square-wave gratings and orientation discrimination task (ODT) used during psychophysical training to determine the time course of contrast learning, control condition was not necessary during this stage of the experiment. (B) Stimulus configuration during main experiment for the psychophysical training sessions, participants received performance feedback through the colour of the fixation square (green-correct, red-incorrect). (C) Stimulus configuration of training in the fMRI scanner, participants performed the same discrimination task as during psychophysical sessions. However stimulus contrast was varied using the method of constant stimuli. (D) Baseline condition belonging to the training sessions in the fMRI scanner, participants performed a central letter discrimination task at fixation. (E) MVPA session in the fMRI scanner. Stimuli in the Trained and Control quadrants were ignored, while participants performed a central letter discrimination task at fixation. (F) The baseline condition for MVPA measurements same as in figure D. (G, H) Details of ODT in Trained quadrant (used during training in psychophysics lab and in scanner). Participants indicated the clockwise $(\mathrm{G})$ or anticlockwise $(\mathrm{H})$ deviation from a never-presented $135^{\circ}$ reference (dashed line). Left/Right arrow keys were used outside the fMRI scanner (correct response illustrated in green); a Go/No-Go paradigm was used during scanning. 
Arrington Research Inc, Scottsdale, USA). A trial started with a blank screen, followed by presentation of a fixation dot at the middle of the screen. In the first $500 \mathrm{~ms}$ of presentation of the fixation dot, participants oriented their eyes towards the fixation dot. This was followed by a $750 \mathrm{~ms}$ period in which accurate fixation would initiate another $250 \mathrm{~ms}$ period in which fixation was to be maintained, followed by a $250 \mathrm{~ms}$ presentation of the grating stimulus. During training in the psychophysics lab, participants responded with their right index (left arrow key on keyboard) or middle finger (right arrow key on keyboard), respectively representing a counter-clockwise or clockwise deviation from a reference orientation $\left(135^{\circ}\right.$ ) (Figure $1 \mathrm{G}, \mathrm{H}$ ). The response window was $750 \mathrm{~ms}$. Participants received feedback on their response by brief colouring of the fixation dot (green for correct, red for incorrect). Stimulus presentation and response recording were performed using Cortex (v.5.9.6; Cortex Software, NIH freeware). Trials with eye position deviations beyond $1.5^{\circ}$ relative to the fixation dot were aborted and replaced by a new randomly generated trial. Trials in which no response was given were also randomly replaced.

\section{Stimuli, stimulus delivery, and responses in scanner}

Stimuli of the same properties were used in the scanner. They were presented using a combination of back projection (Sanyo PLC XT11) on a translucent screen, and a mirror system mounted in front of the eyes on the head cage of the scanner. Stimuli were presented and responses recorded using Presentation software (NeuroBehavioral Systems, NBS, Albany CA USA). Luminances and contrasts of all stimuli and backgrounds were matched by means of careful measurements (Konika Minolta CS 100A luminance meter) in the psychophysics laboratory and scanner. During training in the scanner, participants pushed a button with their right index finger for stimuli representing a counter-clockwise orientation, and withheld a response for stimuli representing a clockwise deviation from the reference orientation $\left(135^{\circ}\right)$. The response window was 750-1750 ms (randomized over trials), participants received no feedback on their response. No eye movement measurements were carried out, but we suggest participants were able to fixate sufficiently accurately also inside the scanner by virtue of training in the psychophysics laboratory (see also MRI Design for MVPA data collection).

\section{Details of behavioural tasks during training in the scanner}

The present paper presents behavioural data obtained during a scanning session at the beginning of training, and at the end of training (respectively referred to as the Pre- and Post learning fMRI sessions). The behavioural data were collected during scanning in three runs while participants were actively engaging in an orientation discrimination task (ODT). Thus, only the behavioural data are used here (Figure $1 \mathrm{C}$ ), and the fMRI data collected in these runs fall outside 
the scope of the present paper. During each run, blocks of square-wave grating stimuli were presented in the upper left and lower right visual field. In half of the participants the location of the stimuli was mirrored. Stimuli were varied both in orientation and contrast (computed as a percentage using Michelson index), Contrast levels varying over blocks between $1,2,3,4,6,8,16$, and $32 \%$ (pseudo randomized, every contrast appearing twice per run). A run contained $16 \times 16$ seconds discrimination blocks (each second presenting a trial, see below) interleaved with 16 seconds fixation blocks, and was divided in two parts by a 32 seconds rest period in the middle of the run where participants were allowed to make eye movements but were instructed not to move their heads (total length of a run 576 seconds).

Within a discrimination trial in a discrimination block, stimuli were presented for 250 ms followed by a 750-1750 ms response period without stimuli. Stimuli were presented, simultaneously in the two locations of the visual field, with contrast level the same in both locations but with orientation randomized between both locations. Specifically, the orientation presented in the Control quadrant was chosen randomly from the same two orientations from which the orientation in the Training quadrant was chosen on a given trial.

Stimulation blocks were interchanged with 'fixation' blocks, in which the participants had to attend to white $T$ and L's presented in the centre of the screen and respond to 1 out of 8 possible different letter/orientation combinations, and in which the grating stimuli were absent (Figure 1C, D). Both in fixation- and in grating discrimination blocks stimuli were pseudo randomized so that targets on average appeared once every 2 seconds. In the fixation task (as in the orientation discrimination task), participants responded to target stimuli with a go-response (button press) and to non-target stimuli with a no-go response (withholding button press).

\section{Details of behavioural task in the psychophysics laboratory}

While performance in the scanner was measured as a function of contrast using a constant stimuli design, performance in the psychophysics lab was measured using staircase thresholds, during which contrast was made dependent on performance at a fixed orientation difference. This fixed orientation difference was determined from preliminary staircase orientation thresholds measured in another unused quadrant (see identification of constant orientation difference, reflecting pre-training performance level).

Participants were trained on the orientation discrimination task in the left or right upper visual field (randomized between participants), using stimuli and stimulus locations as in the fMRI sessions. Contrast JNDs (at fixed orientation difference) were determined using a Wetherill and Levitt staircase that tracked an $84 \%$ correct performance. Measurements were terminated after 14 reversal points or when a total of 120 trials was reached (on average, $\sim 100$ trials were 
performed per staircase), and computed as the geometric mean of the last 10 reversal points.

The training period (comprising sessions in which participants trained in orientation discrimination) had a total average length of 11.3 sessions. These included training sessions in the psychophysics lab and training during fMRI scanning sessions (see identification of constant orientation difference reflecting pre-training performance level). Training was measured using a constant stimuli design in the scanner, and using the staircase procedure in the psychophysics lab. Staircase contrast thresholds measured in the orientation discrimination task decreased from $15-20 \%$ to $3-4 \%$ after reaching the learning asymptote (typically after about 7 training sessions).

\section{MRI data acquisition and preprocessing}

Images were acquired on a 3T Siemens Magnetom Allegra head-scanner (Siemens medical Systems, Erlangen, Germany) located at the Maastricht Brain Imaging Centre (M-BIC). The scanner is equipped with a standard birdcage head-coil. A high resolution anatomical dataset (voxel size $1 \times 1 \times 1 \mathrm{~mm}^{3}$ ) comprising the whole brain was collected at the beginning of each scan session just before the functional scans, in order to facilitate later co-registration of the functional images with the high-resolution structural images, (ADNI, TI $=900 \mathrm{~ms}$, TR $=2250 \mathrm{~ms}$, TE $=2.6 \mathrm{~ms}$, flip angle $=9^{\circ}, 192$ sagittal slices). In each participant 8 runs were acquired. Blood oxygenation changes were monitored using a T2*-sensitive sequence (28 gapless slices, TR/TE, 2,000/30ms; flip angle $90^{\circ}$; square in plane matrix size of $128^{2}$; resulting in isotropic $2 \mathrm{~mm}^{3}$ voxels). Slices were oriented along the calcarine sulcus, and spaced to include upper and lower field of areas from V1 up to at least V4. In a separate session, borders of the visual areas were determined using conventional polar angle mapping (single pie shaped wedge, red/green checkerboard pattern). Each participant completed 4 short runs of 140 volumes. Additionally, two localizer runs of 189 volumes were acquired.

Functional and anatomical images were preprocessed using BrainVoyager QX (Brain Innovation). The steps included sinc-interpolated slice-time correction, 3D-motion correction with sinc-interpolation and linear trend removal. No spatial or additional temporal smoothing was applied. Functional slices were then co-registered to the structural volume and transformed into Talairach space with sinc interpolation.

\section{Localizer stimulus and retinotopic mapping}

Preceding the first fMRI session of the learning paradigm, two localizer runs with high contrast coloured checkerboard stimuli interchanged with fixation blocks (21 blocks, 18 seconds per block, $378 \mathrm{sec}$ total length), were used to determine the exact locations of the regions responding to the positions of the 
stimuli in the two parts of the visual field (trained and control quadrant, $5^{\circ}$ eccentricity, $3^{\circ}$ diameter). The borders of the visual areas were determined using 4 runs of a conventional polar mapping technique (Sereno et al., 1995; Engel et al., 1997; Linden et al., 1999).

\section{MRI Design for MVPA data collection}

Participants' stimulus-driven brain activity was measured during 8 experimental runs of 288 volumes on a single daily session, 9 weeks after the end of training. Blocks of square wave grating stimuli $\left(5^{\circ}\right.$ eccentricity, $5^{\circ}$ diameter, in both trained and control quadrant) of 8 levels of contrast $(1,2,3,4,6,16$ and $32 \%)$ were interchanged with fixation-only blocks. Each run consisted of 16 stimulus blocks, each contrast was shown once for the two orientations; orientation differences depended on personal thresholds. Blocks had a duration of 8 TRs, i.e. 16 seconds, stimuli were presented in a $250 \mathrm{~ms}$ on, $250 \mathrm{~ms}$ off fashion, resulting in 24 stimulus presentations per block. During the run, participants ignored all stimuli and were required to maintain fixation at the centre of the screen $\left(50 \mathrm{~cd} / \mathrm{m}^{2}\right)$. Fixation was ensured by having participants perform a target letter identification task at fixation (see Details of behavioural tasks during training in the scanner); timing of fixation stimuli and grating stimuli was identical (Figure 1E, F. We were not in the opportunity to use fixation control in the scanner. However, participants were highly trained observers (after on average 11 sessions of training), and the MVPA results (see also below) could not have been obtained without accurate fixation. In addition, all stimuli were presented in the experiment during execution of a difficult letter discrimination task at fixation (see Details of behavioural task during training in the scanner), which ensured fixation and locked attention centrally, away from the stimuli.

\section{Analyses}

To investigate in which areas BOLD signal reflected changes associated to perceptual learning, we conducted a multi-voxel pattern analysis (MVPA) on the trained and the control quadrant, using a Region Of Interest (ROI) approach. Multi-subject univariate analyses did not indicate any significant difference $(p<0.05$, Bonferroni corrected) between the average BOLD responses, in the considered ROls, to the different grating orientations at any contrast, and we therefore employed MVPA, due to its increased sensitivity to small distributed effects. MVPA exploits variations in the distribution of orientation channels sampled by individual voxels, leading to specific activity distributions in the population of voxels stimulated by a specific grating orientation (Recanzone et al., 1993). Training-induced changes in these distributions may reflect changes in orientation tuning in orientation selective neurons (Schoups et al., 2001). ROls limited to V1, V2 and V3 areas for both the trained and the control 
quadrant $\left(5^{\circ}\right.$ radius, $5^{\circ}$ eccentricity) were identified based on two independent localizer runs, resulting in 6 separate ROls. Since the selected regions contained different numbers of voxels, we restricted our analysis to 90 voxels per orientation per ROI to avoid a potential dimension bias during MVPA. This was implemented with a univariate preselection: we first estimated a General Linear Model on the training data, and subsequently subselected for each orientation the 90 voxels with highest ranked activity, based on the normalized estimates of the regression coefficients of the GLM, and considered the union of these two sets for the MVPA analysis similarly to (De Martino et al., 2008).

For each of these ROls, we aimed at finding distributed patterns that could reliably discriminate between the left and the right tilted orientation. As only eight samples per orientation per contrast level were available, we increased the available samples per orientation by grouping together contrast levels two by two resulting in 4 sets of contrast levels with a total of 16 samples per orientation per set. Clustering involved the grouping of pairs of contrast levels that were physically closest to each other. Without this grouping, insufficient statistical power was available to conduct the MVPA analyses. We used identical sets of contrast levels for the analyses of the behavioural data (for behavioural analyses at single contrast levels, see chapter 5). We averaged the time-series of each voxel within each block from 3 to 11 volumes after the stimulus onset (first 2 volumes were discarded). To account for changes in baseline, we subtracted from each voxel's average in each trial its baseline, which was estimated in a window comprising four volumes before the block onset. We estimated empirically a different hemodynamic response function in each participant, based on another independent dataset and used it for the univariate preselection.

These voxelwise sets of BOLD signal averaged over time for each orientation at the 4 contrast sets were used by a Support Vector Machine (SVM) - based classifier, for all ROIs in V1, V2 and V3. The SVM determines a discriminative hyperplane that maximally differentiates between the two oriented stimuli. We left a run out for testing and trained the model on the remaining 7 runs. This procedure was repeated for each run, leading to 8 different cross-validation tests, whose accuracy was averaged. To test for statistical significance of our finding, we employed a one sample t-test to determine, for each quadrant and each region, whether the set of accuracies across the different participants were significantly different from chance level $(=50 \%)$. As we tested 6 regions (3 areas for the attended quadrant and 3 areas for the control quadrant), and 4 contrast categories, we applied Bonferroni correction accounting for 24 independent tests.

\section{Results}

Figure 2A shows the learning curve resulting from the data from the 7 psychophysics training sessions that were collected over the course of the experiment. For the trained quadrant, participants show a significant decrease in 
contrast thresholds for which they are able to distinguish between the two stimulus orientations. Asymptotic learning is reached after five sessions, where thresholds have decreased until 3 to $4 \%$ Michelson's contrast. In order to asses behavioural performance changes in the scanner, we compared pre- and post training behavioural data collected during scanning using a paired t-test per contrast interval which revealed a significant training-induced enhancement of discrimination performance, but only at mid-range contrasts 3 and $4 \%$ ( $\mathrm{t}=$ $-4.642, d f=5, p=0.006$; see Figure $2 B$ ). Pairwise comparisons comparing the pre-post learning difference revealed significant differences between contrast levels 1 and $2 \%$ compared to 3 and $4 \%$, but not between the other contrast levels $(p=0.025,0.408,0.272)$. Taken together, learning-induced performance changes in both the scanner and psychophysics laboratory clearly show a similar pattern, with significant learning occurring at contrast levels around 3 and $4 \%$.

To test whether the observed improved discrimination of (on average) an $7.3^{\circ}$ difference (in the scanner) between two gratings at contrasts 3 and $4 \%$ was reflected in an increased dissociation of fMRI responses to these two stimuli, an fMRI experiment was conducted 9 weeks after completing training. The two orientations were shown in blocks, separately at 8 different contrast levels (16 conditions, Methods). To increase statistical power for MVPA, we pooled data from contrasts 1 and $2 \%, 3$ and $4 \%, 6$ and $8 \%$, and 16 and $32 \%$. MVPA was performed on Regions of Interest (ROI) in V1, V2 and V3 corresponding to stimulus locations in trained and control quadrants, identified by two independent localizer runs (Methods). For each visual area, we trained a linear SVM (Christianini and Shawe-Taylor, 2000) on 4 different linear classification problems (2 orientations, 4 contrast sets). We tested whether the decoding accuracies obtained for a specific quadrant were significantly different from the theoretical chance level (50\%) in the participant's pool, by means of one-sample t-tests in each of the 4 grouped contrast conditions, for each area and quadrant.

Remarkably, the only ROI where decoding accuracies were significantly different from chance level (50\%) was in V1 of the trained quadrant (Figure 3), for the 3 and $4 \%$ contrast set, with an average accuracy of $64 \%(t=2.8458$, $\mathrm{df}=5, \mathrm{p}=0.036$; Bonferroni-corrected, for 24 tests). This is precisely the condition in which training had resulted in significant increases in discrimination performance. Thus, MVPA did not reveal differences between the two trained orientations in any of the stimulus conditions, and in any of the visual areas tested, except in V1 for the contrasts at which the greatest increase in behavioural performance had taken place.

\section{Discussion}

Our results revealed tantalizing evidence of long-lasting, training-induced plastic changes in human V1, 9 weeks after completing training in a visual perceptual task. Our behavioural data show that contrast thresholds in an orientation discrimination task with fixed orientation difference decrease most at interme- 

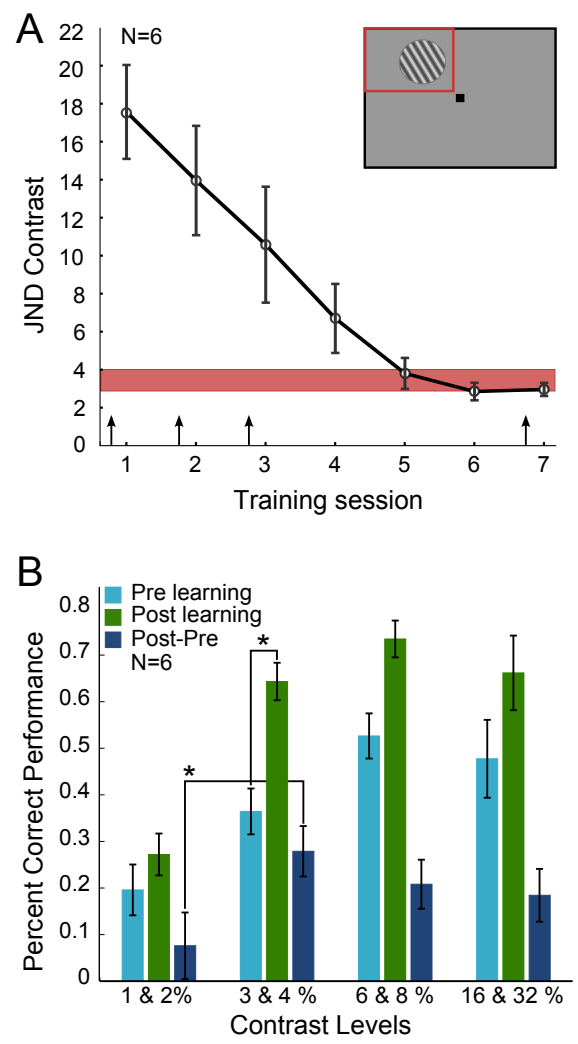

Figure 2. Behavioural training effects. (A) Learning curve resulting from psychophysical training sessions, the just noticeable difference [(JND)] plotted as a function of session. The arrows indicate the timing of the fMRI training sessions (some participants trained on extra sessions depending on when they reached asymptotic performance). Contrast performance saturated around 3 to $4 \%$. (B) Behavioural performance during fMRI sessions. The bar graph shows $\%$ correct performance per contrast category for Pre- (light blue) and Post (green) training fMRI sessions, as well as their difference (dark blue) ( 576 trials per bar). A t-test per contrast interval between pre and post training performance revealed a significant increase in performance but only for the 3 and $4 \%$ interval $(\mathrm{t}=-4.642, \mathrm{df}=5, \mathrm{p}=0.006$, pairwise comparisons comparing the pre-post learning difference revealed significant differences between contrast levels 1 and $2 \%$ compared to 3 and $4 \%$, but not between the other contrast levels $(p=0.025$, $0.408,0.272)$. 


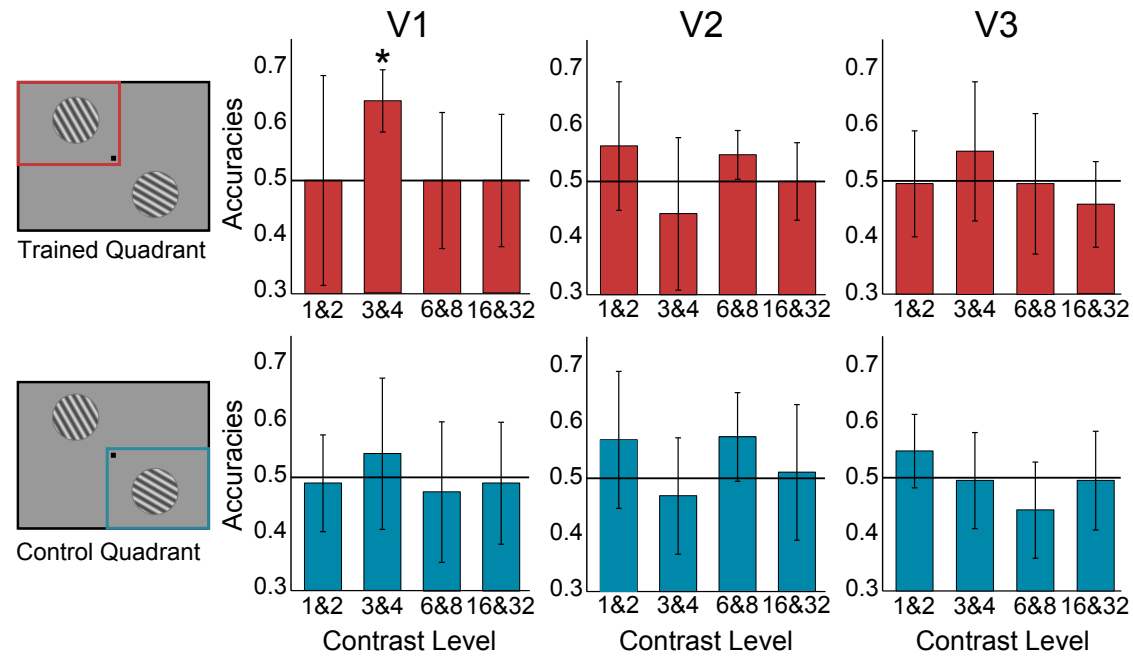

Figure 3. Enhanced difference between brain-activation patterns elicited by grating stimuli used during ODT. The figure shows the resulting decoding accuracies for the trained quadrant (red frame), and the passively exposed control quadrant (blue). The columns show the accuracies for the three visual areas (V1, V2 and V3), each depicting the accuracies for the 4 sets of contrasts $(1 \& 2,3 \& 4,6 \& 8$ and $16 \& 32 \%)$. Asterisk shows accuracy significantly higher than chance $(0.5)(t=2.8458, d f=5, p=0.036)$. 
diate contrasts ( 3 and $4 \%$ ) Remarkably, at those contrasts, the two orientations used for training $\sim 8^{\circ}$ difference) could be successfully classified by MVPA of fMRI data after training, but not before training. Remarkably, the successful classified orientation difference (80) was much smaller than that classified in other studies (typically $90^{\circ}$ ) (Haynes and Rees, 2005), suggesting significant training-induced cortical plasticity. The results (Figure 3) show that the memory trace was present only in $\mathrm{V} 1$, only in the trained location, and only in the stimulus conditions showing the training effects. Moreover, the fMRI responses were measured while participants ignored the stimuli and performed a demanding task at fixation. This excludes the possibility that our findings were an artefact of attention or effort, or differences in these variables among contrast conditions (Yotsumoto et al., 2008). Hence, our data suggest that V1 contributes to the long-term maintenance of memory traces induced by visual skill learning.

The finding of greatest training-induced improvements in discrimination performance at intermediate to low contrasts is reminiscent of neurophysiological studies of attention that showed the largest enhancement of neural response exactly for that category of contrasts (Reynolds et al., 2000). These enhanced responses for a specific sub range of contrasts were described as a shift of the contrast curve towards lower contrasts, in line with input gain theories of attention (Luck et al., 2000; Reynolds et al., 2000; Martinez-Trujillo and Treue, 2002; Reynolds and Chelazzi, 2004). These enhanced responses, bringing neural responses to low contrast stimuli clearly above threshold, may contribute to better discriminability of the stimuli (Lu and Dosher, 1998; Carrasco et al., 2000; Cameron et al., 2002; Carrasco et al., 2004). The enhanced neural responses induced by attention to low contrast stimuli may become hard-wired by visual skill learning, leading to a more permanent enhancement of discriminability of low contrast stimuli. This may result in better classification in V1 by MVPA of the two orientations at 3 and $4 \%$ after training.

However, this interpretation has some limitations. To statistically asses claims of specificity of the effect for V1 and the 3 and 4\% contrast level, we would have to additionally perform a set of statistical analyses comparing classification accuracies between quadrants, visual areas and contrast levels, which is difficult because of the small number of participants $(\mathrm{N}=6)$ and the resulting lack of statistical power. More importantly, the question can be asked why the behavioural classification of the orientations at about $70 \%$ correct (for behaviour during scan sessions) reached after training at contrasts 3 and $4 \%$ is paired by successful classification of these orientations by the MVPA classifier, whereas similar levels of behavioural classification between orientations around $70 \%$ correct before and after training at higher contrasts are not associated with successful MVPA classification. In interpreting this apparent inconsistency, it is important to keep in mind that prior studies that used MVPA of cortical fMRI responses to oriented stimuli, typically used stimuli with larger orientation differences (Kamitani and Tong, 2005). Some studies have shown 
for these large differences that classification accuracy increased with increasing contrast (Smith et al., 2011). However, the features of the patterns used by MVPA to distinguish very small differences in orientation may be different, and it is possible that saturation phenomena take place at fairly low contrasts, so that successful MVPA classification of stimuli that differ little in orientation at higher contrast might become difficult. Systematic studies of MVPA classification performance as a function of contrast and orientation difference are currently lacking, but they could shed light on the findings of the present study.

An alternative possibility is that the behavioural training, which has its largest effect for contrasts of 3 to $4 \%$, led to contrast-specific modifications of the stimulus representations that helped the MVPA classifier to distinguish the orientations. This is not implausible as several studies have demonstrated contrastspecific visual perceptual learning (Yu et al., 2004; Zhang et al., 2008). These training-induced alterations of the stimulus representations specifically for 3 and $4 \%$ contrast stimuli, may have been disproportionally effective in creating distinguishable distributed representations for the two orientations that could be captured by MVPA. For higher contrast levels these alterations might have been less pronounced, thereby explaining our results. Nonetheless this remains speculative, and requires more knowledge about both the underlying mechanisms of plasticity and the relationship between neuronal patterns and fMRI patterns (which are captured with MVPA, (Formisano and Kriegeskorte, 2012). Ultra high field follow up studies might be decisive in shedding light on these issues.

Despite some open question regarding the interpretation of the data, we suggest that the finding of a significant increase in MVPA classification accuracy in V1 at the very contrasts (3 and 4\%) for which we observed significant traininginduced enhancements of performance is not a random finding. The idea that these highly specific changes in stimulus representation occur only in primary visual cortex is in line with early theoretical proposals on the neural substrate of visual perceptual learning (Karni and Sagi, 1991, 1993; Karni and Bertini, 1997). The finding that more than 2 months after completion of training, neural activity patterns in parts of V1 corresponding to the trained quadrant still carry information about trained stimuli gives a tantalizing indication that human V1 indeed plays a privileged role in the storage of lasting memory traces related to specific visual expertise.

\section{References}

Cameron EL, Tai JC, Carrasco M (2002) Covert attention affects the psychometric function of contrast sensitivity. Vision Res 42:949-967.

Carrasco M, Penpeci-Talgar C, Eckstein M (2000) Spatial covert attention increases contrast sensitivity across the CSF: support for signal enhancement. Vision Res 40:1203-1215.

Carrasco M, Ling S, Read S (2004) Attention alters appearance. Nat Neurosci 7:308-313.

Christianini N, Shawe-Taylor J (2000) An Introduction to Support Vector Machines. 


\section{Lasting memory traces of visual skill learning in human V1}

Cambridge: Cambridge University Press.

De Martino F, Valente G, Staeren N, Ashburner J, Goebel R, Formisano E (2008)

Combining multivariate voxel selection and support vector machines for mapping and classification of fMRI spatial patterns. Neuroimage 43:44-58.

Engel SA, Glover GH, Wandell BA (1997) Retinotopic organization in human visual cortex and the spatial precision of functional MRI. Cereb Cortex 7:181-192.

Fahle M (2005) Perceptual learning: specificity versus generalization. Curr Opin Neurobiol 15:154-160.

Formisano E, Kriegeskorte N (2012) Seeing patterns through the hemodynamic veil - The future of pattern-information fMRI. Neuroimage.

Haynes JD, Rees G (2005) Predicting the orientation of invisible stimuli from activity in human primary visual cortex. Nat Neurosci 8:686-691.

Kaas JH, Krubitzer LA, Chino YM, Langston AL, Polley EH, Blair N (1990) Reorganization of retinotopic cortical maps in adult mammals after lesions of the retina. Science

248:229-231.

Kamitani Y, Tong F (2005) Decoding the visual and subjective contents of the human brain. Nat Neurosci 8:679-685.

Karni A (1996) The acquisition of perceptual and motor skills: a memory system in the adult human cortex. Brain Res Cogn Brain Res 5:39-48.

Karni A, Sagi D (1991) Where practice makes perfect in texture discrimination: evidence for primary visual cortex plasticity. Proc Natl Acad Sci U S A 88:4966-4970.

Karni A, Sagi D (1993) The time course of learning a visual skill. Nature 365:250-252.

Karni A, Bertini G (1997) Learning perceptual skills: behavioral probes into adult cortical plasticity. Curr Opin Neurobiol 7:530-535.

Kastner S, De Weerd P, Desimone R, Ungerleider LG (1998) Mechanisms of directed attention in the human extrastriate cortex as revealed by functional MRI. Science 282:108-111.

Law CT, Gold JI (2008) Neural correlates of perceptual learning in a sensory-motor, but not a sensory, cortical area. Nat Neurosci 11:505-513.

Linden DE, Kallenbach U, Heinecke A, Singer W, Goebel R (1999) The myth of upright vision. A psychophysical and functional imaging study of adaptation to inverting spectacles. Perception 28:469-481.

Lu ZL, Dosher BA (1998) External noise distinguishes attention mechanisms. Vision Res 38:1183-1198.

Luck SJ, Woodman GF, Vogel EK (2000) Event-related potential studies of attention. Trends Cogn Sci 4:432-440.

Martinez-Trujillo J, Treue S (2002) Attentional modulation strength in cortical area MT depends on stimulus contrast. Neuron 35:365-370.

Mur M, Bandettini PA, Kriegeskorte N (2009) Revealing representational content with pattern-information fMRI-an introductory guide. Soc Cogn Affect Neurosci 4:101-109. Norman KA, Polyn SM, Detre GJ, Haxby JV (2006) Beyond mind-reading: multi-voxel pattern analysis of fMRI data. Trends Cogn Sci 10:424-430.

Recanzone GH, Merzenich MM, Dinse HR (1992) Expansion of the cortical representation of a specific skin field in primary somatosensory cortex by intracortical microstimulation. 


\section{References}

Cereb Cortex 2:181-196.

Recanzone GH, Schreiner CE, Merzenich MM (1993) Plasticity in the frequency

representation of primary auditory cortex following discrimination training in adult owl monkeys. J Neurosci 13:87-103.

Reed A, Riley J, Carraway R, Carrasco A, Perez C, Jakkamsetti V, Kilgard MP (2011) Cortical map plasticity improves learning but is not necessary for improved performance. Neuron 70:121-131.

Reynolds JH, Chelazzi L (2004) Attentional modulation of visual processing. Annu Rev Neurosci 27:611-647. Reynolds JH, Pasternak T, Desimone R (2000) Attention increases sensitivity of V4 neurons. Neuron 26:703-714.

Schoups A, Vogels R, Qian N, Orban G (2001) Practising orientation identification improves orientation coding in V1 neurons. Nature 412:549-553.

Sereno MI, Dale AM, Reppas JB, Kwong KK, Belliveau JW, Brady TJ, Rosen BR, Tootell RB (1995) Borders of multiple visual areas in humans revealed by functional magnetic resonance imaging. Science 268:889-893.

Smirnakis SM, Brewer AA, Schmid MC, Tolias AS, Schuz A, Augath M, Inhoffen W, Wandell BA, Logothetis NK (2005) Lack of long-term cortical reorganization after macaque retinal lesions. Nature 435:300-307.

Smith AT, Kosillo P, Williams AL (2011) The confounding effect of response amplitude on MVPA performance measures. Neuroimage 56:525-530.

Xiao LQ, Zhang JY, Wang R, Klein SA, Levi DM, Yu C (2008) Complete transfer of perceptual learning across retinal locations enabled by double training. Curr Biol 18:1922-1926.

Yotsumoto Y, Watanabe T, Sasaki Y (2008) Different dynamics of performance and brain activation in the time course of perceptual learning. Neuron 57:827-833.

Yu C, Klein SA, Levi DM (2004) Perceptual learning in contrast discrimination and the (minimal) role of context. J Vis 4:169-182.

Zhang J, Kourtzi Z (2010) Learning-dependent plasticity with and without training in the human brain. Proc Natl Acad Sci U S A 107:13503-13508.

Zhang JY, Kuai SG, Xiao LQ, Klein SA, Levi DM, Yu C (2008) Stimulus coding rules for perceptual learning. PLoS Biol 6:e197. 



\section{Neural correlates of contrast learning in the human visual cortex}

based on: Been, M.M., Jans, B., and Peter De Weerd, P., Neural correlates of contrast learning in the human visual cortex. In Preparation. 


\section{Abstract}

The neural mechanisms underlying visual skill learning remain poorly understood. In particular, there is a persistent debate about the role of primary visual cortex (V1) compared to higher-level areas in the visual system. Moreover, the potentially varying contribution of different visual areas in the course of learning is virtually unexplored. We devised a task in which participants were trained to perform an orientation discrimination task at a fixed orientation difference while contrast was made dependent on performance using a staircase procedure. Participants were trained in 6-9 (average 6.7) sessions in the psychophysics laboratory, which were interspersed with 4-6 (average 4.7) fMRI sessions. Psychophysical contrast thresholds determined in this task decreased strongly, and proved highly position specific, in line with a contribution of lowlevel visual areas. However, behavioural data collected during fMRI showed less clear evidence of learning, and we found only limited evidence for plasticity in V1 or other low-level visual areas. Moreover, this evidence for plasticity was present only for a comparison of pre-training and post-training fMRI sessions. We discuss these findings in the context of current literature, of potential limitations of analysis performed so far, and indicate future perspectives.

\section{Introduction}

Perceptual learning involves improvements in sensory abilities and gains in performance induced by extensive sensory experience or training. Once a skill is acquired, it is retained over a long period of time, which suggests that training can induce long term changes. In particular, perceptual learning is often regarded as a manifestation of experience-dependent plasticity in sensory systems (Karni and Sagi, 1991; Gilbert et al., 2001; Watanabe et al., 2001; Fahle and Poggio, 2002), yet the underlying neural mechanisms remain unclear.

One of the major objectives of neuroscience research is to link behaviour to neural mechanisms. One of the characteristic features of (perceptual) skill learning is its slow time course. Hence, to study neural correlates of perceptual learning, it is important to study changes in neural activity monitored at regular time intervals along the entire learning curve. Surprisingly, with a few exceptions (see Discussion), studies of perceptual learning have been limited to comparisons of neural correlates associated with pre- and post-training performance. A major goal of the present study was to track changes in fMRI activity in all areas activated by the chosen stimuli in the course of learning, using a grating orientation discrimination task as a learning paradigm. The rationale for our study was to distinguish different theories of perceptual learning, which make different predictions for the involvement of areas at different levels of the visual hierarchy at different levels of expertise. In the training paradigm we used, learning was mediated by attention to the stimulus, and we therefore based our predictions solely on theories related to attentional learning. 
There are two main theoretical proposals regarding the locus and timing of plastic changes induced by perceptual learning. The first emphasizes changes in filtering properties at the lowest levels of the visual hierarchy. In the Lowest Level Theory (LLT), (Karni and Bertini, 1997), it is predicted that the formation of a memory trace will occur at the lowest level of the information processing stream where the best neuronal processing capability is available for the representation of the stimulus parameters relevant for task performance. The plastic changes at the relevant lowest processing levels are hypothesized to take place when the demands in the task exceed processing capacity. From that moment on, session-to-session performance increments become slow, and learning becomes asymptotic. Thus, perceptual expertise and corresponding low-level plastic changes are thought to develop relatively late in learning. The other main proposal focuses on the fine-tuning of readout mechanisms. Here, it is assumed that the expertise that is built up during perceptual learning is related to changes in read-out from low-level sensory cortex, and not in modification of filtering properties through plasticity in local circuits. According to Reversed Hierarchy Theory (RHT) (Ahissar and Hochstein, 2002, 2004; Ahissar et al., 2009), expert performance during the asymptotic phase of learning is due to changes in connectivity to read out information from areas increasingly lower in the visual hierarchy. Recent studies have demonstrated that perceptual learning can occur without obvious neural correlates of plasticity in relevant low-order motor areas (Law and Gold, 2008) or primary visual cortex (Zhang and Kourtzi, 2010).

RHT and LLT make different predictions about the location and timing of changes during perceptual learning involving oriented stimuli. According to a strong prediction based on LLT, in our task (an orientation discrimination task, in which performance is measured as a function of contrast) changes would occur only in V1, late in learning, whereas a strong hypothesis based on RHT or read-out would predict changes in high-level areas first, followed by changes in increasingly lower-level areas, possibly involving V1. Some recent evidence (Yotsumoto et al., 2008) suggests that the time course of learning-induced plasticity in visual tasks is uncoupled from (precedes) the changes in behavioural performance. Except for that study, and the above theoretical considerations, there is little basis to propose detailed hypotheses on the precise time course of plastic changes in different visual cortical areas in the course of skill learning, and it is therefore important to investigate this question.

Even without considering the lack of information on the timing of traininginduced plasticity, findings on the involvement of different levels of the visual system in visual perceptual learning at the end of training are contradictory. On the one hand, there are findings that perceptual learning in the visual system can be very specific for stimulus location or orientation (Ball and Sekuler, 1987; Karni and Sagi, 1991; Schoups et al., 1995; Ahissar and Hochstein, 1996). These specificities are usually interpreted as indications of contributions of low-level visual cortical areas to perceptual learning. In line with plasticity 
at low-levels in the visual system, Schoups et al. (2001) reported an increase in steepness of the slopes of tuning curves for trained orientations after training on an orientation identification task in the primary visual cortex (V1) of rhesus monkeys. On the other hand, several physiological studies have not been able to find any changes in the responsiveness of V1 and V2 neurons that could account for the observed perceptual learning. For example Yang and Maunsell (2004) recorded in areas V1 up to V4 and found learning-induced narrowing of orientation tuning only in V4. Raiguel et al., (2006) also found an increase in orientation selectivity in V4, similar to Schoups et al., (2001)'s findings in V1. So for simple stimuli, some studies do not find evidence of learning-induced plasticity in V1/V2, while other studies do suggest plasticity in V1. Taken together these results illustrate the uncertainty related to the existence of training-induced plasticity both in V1 and higher-level visual areas.

There are several fMRI studies relevant for the design of our own study that also have addressed the question of training-induced plasticity in low-level visual cortex in simple visual tasks. They have shown interesting results, in terms of the levels of the visual system involved and the timing of involvement in the time course of learning. Schwartz et al. (2002) let participants train on a monocular texture discrimination task, 24 hours before scanning. During the fMRI session the participants had to perform the same task using the trained and untrained eye in an alternating sequence, and they showed increased V1 activity selectively for the trained eye. A second fMRI study by Vaina et al. (1998) set out to try to characterize the rapid learning of direction discrimination in global motion stimuli. The results indicated that learning occurred very fast over less than 300 trials and was direction and location specific. MT showed a significant increase in activation over the course of runs, but no increase in activity was found for V1. A third interesting fMRI study is that of Furmanski et al., (2004). They exploited the fact that oblique gratings require higher contrast for detection than gratings oriented at cardinal axes (oblique effect, (Appelle, 1972). They trained their participants specifically on detecting oblique gratings, and found that after training they reached a performance level similar to that for horizontal gratings. In addition, training increased the response of V1 to the oblique orientation, so that BOLD responses to the two stimuli became close to equal, whereas before training, V1 responses were higher for the horizontal stimulus. The three studies indicate that the amplitude of the fMRI signal in the visual cortex increases as a function of the amount of perceptual learning. For texture and motion stimuli, the activity increase occurred after limited training, while training to increase contrast sensitivity required more training to increase V1 activity.

Visual perceptual learning has an intimate relationship with attention. An important function of attention as proposed by Reynolds and colleagues (2000) is to enhance the processing of weak stimuli; they found that attention selectively increased activations for low to medium contrasts, thereby improving the stimulus' detectability selectively for weak stimuli, which is in line with the in- 
put gain model (Reynolds et al., 2000). Thus, one possible prediction derived from a possible link between visual perceptual learning and attention is that upon completion of visual skill learning with stimuli along a range of contrasts, stimuli at low to mid-range contrasts presented outside the focus of attention might yield an automatically enhanced neural response, as if the stimuli were being attended. According to this hypothesis, skill learning would lead to an automatic incorporation of the attentional effect in the response to trained stimuli, and the response to trained stimuli would show input gain (compared to a baseline of untrained stimuli). Skill learning would become visible as a form of 'frozen attention'.

Here, we used fMRI to test the time-course of learning and the concept of learning being a form of frozen attention, in a visual perceptual learning paradigm. Because some studies might have failed to detect memory-related plasticity due to their choice of stimuli (Zhang and Kourtzi, 2010), or insufficient training (Xiao et al., 2008), we used square wave gratings, known to drive V1 neurons well, and trained participants on an orientation discrimination task in a psychophysics setting $(\mathrm{N}=7)$ until they reached asymptotic performance. During training, the orientation difference was kept constant (as determined by personal thresholds, see Methods) and performance was measured by means of contrast thresholds. To assess the effect of learning on the contrast response curve and the involvement of areas along the visual hierarchy over the timecourse of learning, we scanned participants multiple times along the time course of learning while showing grating stimuli of eight different contrast levels. Our paradigm was designed to probe the contribution of cortical areas along the visual hierarchy in a longitudinal design spanning multiple weeks, and to test the influence of learning on the contrast response curves, which we hypothesized might show a form of training-induced input gain compared to matching untrained stimuli. The comparison between contrast curves for trained and untrained stimuli was done using passive stimulation (without attention directed to the stimuli). This is required to ensure that the effects measured are not confounded by attention, and to separate effects of training from effects of passive exposure, as some studies have indicated that learning can occur through passive unattended stimulation (Godde et al., 2000; Dinse et al., 2006; Frenkel et al., 2006).

\section{Materials and Methods}

\section{Participants}

All seven (6 female, 1 male, age 22-35) participants had normal or corrected to normal visual acuity, gave written informed consent for all parts of the experiment, and were debriefed following completion of data acquisition (same participants as in chapter 4). Participants were financially compensated for their participation. Participants were carefully selected on their motivation to 
participate in a long-term learning study. Before the start of the study, every participant was first tested on a series of orientation training sessions in order to assess the orientation threshold to be used in the main experiment. Moreover, we aimed to asses the participants' fixation ability as well as the course and speed of learning in order to plan the amount and distribution of fMRI sessions over time. This assessment we called pre-training and was done in a quadrant in the lower visual field that is not used in the main experiment (Fig 1a).

\section{MRI data acquisition}

Images were acquired on a 3T Siemens Magnetom Allegra head-scanner (Siemens medical Systems, Erlangen, Germany) located at the M-BIC institute in Maastricht, The Netherlands. The scanner is equipped with a standard birdcage head-coil. A High resolution Anatomical dataset (voxel size $1 \times 1 \times 1 \mathrm{~mm}^{3}$ ) comprising the whole brain was collected at the beginning of each scan session just before the functional scans, in order to facilitate later co-registration of the functional images with the high-resolution structural images, (ADNI, TI $=900$ $\mathrm{ms}, \mathrm{TR}=2250 \mathrm{~ms}, \mathrm{TE}=2.6 \mathrm{~ms}$, flip angle $=9^{\circ}, 192$ sagittal slices). In each participant 7 experimental runs were acquired, 2 passive runs followed by 3 active runs and concluding with 2 passive runs (see Stimuli and experimental design in the fMRI scanner). Blood oxygenation changes were monitored using a T2*-sensitive sequence (28 gapless slices, TR/TE, 2,000/30ms; flip angle $90^{\circ}$; square in plane matrix size of $128^{2}$; resulting in isotropic $2 \mathrm{~mm}^{3}$ voxels). Slices were oriented along the bank of the calcarine sulcus, and spaced to include upper and lower field of areas from V1 up to at least V4.

In a separate session, borders of the visual areas were determined using conventional polar angle mapping (single pie shaped wedge, red/green checkerboard pattern) (Sereno et al., 1995; Goebel et al., 1998). Each participant completed 4 short runs of 140 volumes. Additionally, two localizer runs of 189 volumes were acquired, high contrast checkerboard stimuli interchanged with fixation blocks (21 blocks, 18 seconds per block, 378 sec total length) were used to determine the exact locations of the regions corresponding to the positions of the stimuli in the two parts of the visual field (trained and control quadrant, $5^{\circ}$ eccentricity, $5^{\circ}$ diameter).

\section{Stimuli and procedure}

\section{Psychophysical design}

\section{Stimuli, stimulus delivery in psychophysics laboratory}

FMRI sessions were separated by multiple psychophysical training sessions. Psychophysical training continued until participants reached asymptotic learning, which resulted on average in a total of 6.7 (range 6 to 9) psychophysical ses- 
sions, interspersed with on average 4.7 (range 4 to 6 ) fMRI scanning sessions. During training sessions, participants were trained on an orientation discrimination task with performance measured in contrast thresholds, in the left or right upper visual field (for details, see Task in psychophysics laboratory, and Fig. 1B).

Stimuli consisted of square wave gratings (1c/deg spatial frequency, presented on a PC monitor 19 inch TFT screen, $60 \mathrm{~Hz}$ refresh rate, $1280 \times 1024$ pixel resolution, $5^{\circ}$ diameter, $5^{\circ}$ eccentricity, phase-randomized). The experiment was conducted in a dimly-lit room. The average luminance of stimuli and of the grey background was kept constant with luminance set to $56 \mathrm{~cd} / \mathrm{m}^{2}$. Participants placed their head in a chin rest to stabilize their heads position relative to the monitor. Eye position was monitored with an infrared eye camera (60 Hz sampling rate Viewpoint Eye Tracker v.2.8.3, Arrington Research Inc, Scottsdale, USA). A trial started with a blank screen, followed by presentation of a fixation dot at the middle of the screen. In the first $500 \mathrm{~ms}$ of presentation of the fixation dot, participants oriented their eyes towards the fixation dot. This was followed by a $750 \mathrm{~ms}$ period in which accurate fixation would initiate another $250 \mathrm{~ms}$ period in which fixation was to be maintained, followed by a $250 \mathrm{~ms}$ presentation of the grating stimulus.

\section{Task in Psychophysics laboratory}

In the task used in our experiments, the efficiency of grating orientation discrimination was measured at a constant orientation difference, and grating contrast was made dependent on performance using a staircase procedure (Wetherill and Levitt, 1965). As the time course of contrast-training in an orientation discrimination task at constant orientation difference was unknown, learning curves were first determined in the psychophysics laboratory for one of the two lower quadrants of the visual field (Figure 1A, lower quadrant picked at random in different participants). This training phase is referred to as 'pre-training'. The pre-training started by estimating personal orientation thresholds at an oblique reference orientation $\left(135^{\circ}\right)$ and a relatively high contrast $(16 \%)$ in one or two sessions of orientation training (8 thresholds estimating $84 \%$ correct with a Wetherill and Levitt, 1965 staircase procedure). This resulted in average thresholds of 7.5 degrees. This value was then divided by 2 to estimate the hypothetical performance on the next day, resulting in an average orientation difference of 3.7 degrees. At that orientation difference, training was continued by performing a staircase procedure wherein the orientation discrimination difficulty depended on the contrast of the grating (84\% correct staircase procedure, (Wetherill and Levitt, 1965). During training in the psychophysics laboratory, participants had to indicate a counter-clockwise or clockwise deviation (from a never shown 135 degree reference orientation) by pressing either with their right index (left arrow key on keyboard) or middle finger (right arrow key on keyboard), respectively (Fig $1 \mathrm{C}$ ). The response window was $750 \mathrm{~ms}$. Partic- 

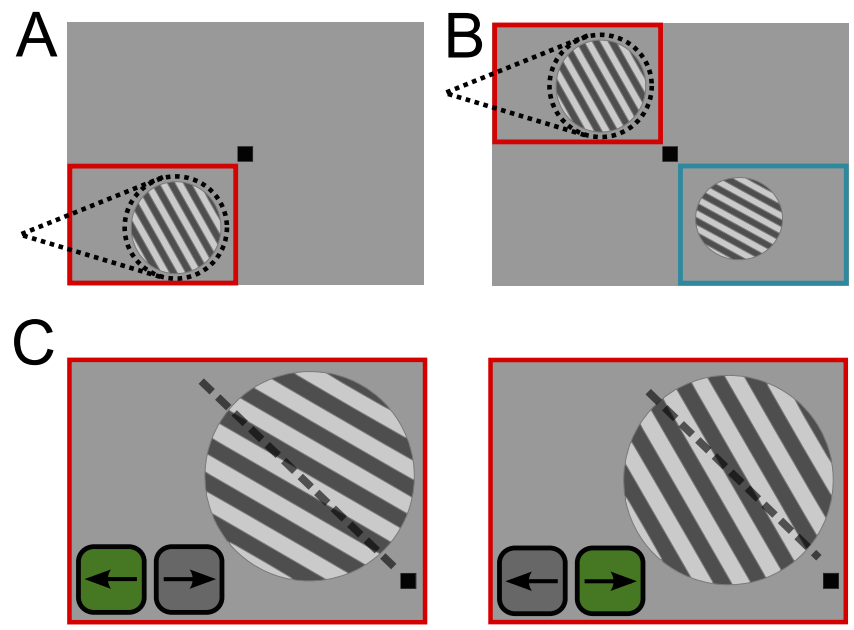

Figure 1. Experimental design. Participants fixated at the centre of the screen, covertly attended and discriminated the stimulus in one of the visual field quadrants, depending on the experimental condition (trained quadrant, red frame), and ignored the stimulus in the diametrically opposite quadrant (control quadrant, blue frame). Cone shows allocation of attention. (A) Square-wave gratings and orientation discrimination task (ODT) used during psychophysical training to determine the time course of contrast learning, the control condition was not necessary during this stage of the experiment. (B) Stimulus configuration during the main experiment for the psychophysical training sessions, participants received performance feedback through the colour of the fixation square (green-correct, red-incorrect). (C) Details of the orientation discrimination task in trained quadrant (used during training in both the psychophysics laboratory and in the fMRI scanner). Participants indicated the clockwise or anticlockwise deviation from a never-presented $135^{\circ}$ reference (dashed line). Left/Right arrow keys were used outside the fMRI scanner (correct response illustrated in green).

ipants received feedback on their response by brief colouring of the fixation dot (green for correct, red for incorrect). Trials with eye position deviations beyond $1.5^{\circ}$ relative to the fixation dot were aborted and replaced by a new randomly generated trial. Trials in which no response was given were randomly replaced. Staircase measurements were terminated after 14 reversal points or when a total of 120 trials was reached (on average, 100 trials were performed per staircase). The just noticeable difference (JND) was then computed as the geometric mean of the last 10 reversal points. Stimulus presentation and response recording were performed using Cortex (v.5.9.6; Cortex Software, NIH freeware). 
B

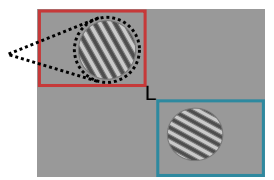

C

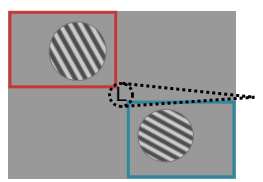

$\mathrm{D}$

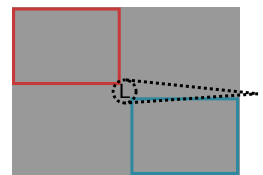

Figure 2. fMRI paradigm. (A) Overview of a typical run, in which experimental blocks are interchanged with fixation blocks. Each contrast level was presented twice in every run, once before and once after the break, the order of contrast levels was pseudo randomized. Every fMRI session consisted of 7 runs, which came in a sequence of 2 passive runs, 3 active runs, and 2 passive runs. Numbers refer to presentation blocks at a given \% Michelson contrast, and $F$ refers to blocks in which fixation task is performed. (B) Experimental task during the active runs. Participants kept central fixation, while covertly attended the grating stimulus in the upper visual field (choice of left or right upper quadrant was randomized among participants) in the context of an orientation discrimination task. Participants were instructed to ignore the stimuli that were presented in the lower visual field. (C) Experimental task during the passive runs. Stimulus configuration during passive runs was identical to that of active runs. During passive runs participants performed an attentional demanding fixation task, while ignoring contrast stimuli that were presented in two quadrants of the visual field (run number 1,2,6,7). (D) Experimental task during baseline periods. In both passive and active runs, experimental blocks were interleaved with fixation only blocks (baseline); here the grating stimuli were absent, where they performed the central fixation task. Cone in dashed line indicates location of attention (which in B was covert).

\section{Stimuli and experimental design in the fMRI scanner}

Stimuli of the same properties as those in the psychophysics design were used in the $\mathrm{fMRI}$ scanner. They were presented using a combination of back projection (Sanyo PLC XT11) on a translucent screen, and a mirror system mounted in front of the eyes on the head coil of the scanner. Stimuli were presented and responses recorded using Presentation software (NeuroBehavioral Systems, NBS, Albany CA USA). Luminance and contrasts of all stimuli and backgrounds were matched by means of careful measurements (Konika Minolta CS 100A luminance meter) in the psychophysics laboratory and fMRI scanner $\left(50 \mathrm{~cd} / \mathrm{m}^{2}\right)$.

Each fMRI session consisted of seven runs, during which blocks of squarewave grating stimuli were presented in the upper left and lower right visual field (in one half of participants), or upper right and lower left visual quadrant (on the other half of participants). Gratings were presented in a circular aperture with a radius of $5^{\circ}$, and centered $5^{\circ}$ from fixation. The gratings had a spatial frequency of 2 cycles/degree and contrast level varying over blocks between 
$1,2,3,4,6,8,16$, and $32 \%$ Michelson contrast (pseudo randomized, every contrast appearing twice per run). Gratings deviated clockwise or anticlockwise from a $135^{\circ}$ reference orientation at a fixed orientation difference. The fixed orientation difference between the stimuli equaled the personal threshold measured at the beginning of the pre-training in the psychophysics laboratory $\left(7.5^{\circ}\right)$. Hence, the orientation difference used in the scanner was twice as high as during pre-training in the psychophysics laboratory (see Tasks in Psychophysics laboratory). As indicated by pilot experiments, this was necessary to keep performance levels in the scanner similar to those of the psychophysical measurements. Blocks of grating were interchanged with 'fixation' blocks (to serve as a baseline).

Due to the length of the runs, every run had a 16 volume break where a red square was presented at fixation, during which the participants were instructed to relax their eyes but keep movement to an absolute minimum (Fig 2A). The grating stimuli were presented in two types of runs in which the stimulus configurations stayed the same, but in which participants carried out different tasks. We refer to these two types of runs as Active and Passive runs.

The 7 runs in each fMRI session were divided into two passive runs, followed by three active runs and then two more passive runs. During active runs participants kept central fixation, and covertly attended the grating stimulus in the upper visual field (left/right, randomized between participants. Here, participants performed an orientation discrimination task (orientation was kept constant, contrast was varied) while ignoring the stimuli presented in the lower visual field (see Fig $2 \mathrm{~B}$ ). During active runs, the $\mathrm{T} / \mathrm{L}$ stimuli at fixation purely served as an anchor to maintain fixation. During passive runs, participants attended to white $T$ and L's presented in the centre of the screen while ignoring the gratings in both quadrants (Fig 2C)(details in next section). In both active and passive runs, blocks of grating presentations (16s) were interspersed with $\mathrm{T} / \mathrm{L}$ blocks in which the grating stimuli were absent and in which only the $\mathrm{T} / \mathrm{L}$ task was performed at fixation (figure 1D). The total length of a single run was 576 seconds.

Importantly, each stimulus presentation in the trained quadrant was paired with a stimulus presentation in the control (untrained) quadrant (stimuli matched in contrast, but there was no correspondence in their clockwise/anticlockwise deviation from the reference orientation). These paired presentations were done both during training in the psychophysics laboratory and during scanning.

\section{Details of tasks performed in fMRI scanner}

The task performed with the gratings during active runs was different from the task performed in the psychophysics laboratory (for details, see section Task in Psychophysics laboratory). In the scanner, participants were instructed to respond to one of the two grating orientations (the orientation deviating anti-clockwise from the $135^{\circ}$ reference orientation). However, stimulus pre- 
sentations continued even if the participants did not give a response, which happened frequently at the very lowest contrasts especially at the beginning of learning (i.e., there was no forced response). Hence, performance could vary from $0 \%$ correct (if participants were unable to detect the stimulus) to $100 \%$ correct (if participants did detect the stimuli and discriminated them correctly). Performance in this task was analyzed using signal detection theory (see Fig. 7).

Within a block, the orientation of the stimuli was pseudo randomized so that grating targets (gratings deviating anticlockwise from the reference) appeared on average every 2 seconds. Note that in the T/L fixation task, participants responded to 1 out of 8 possible different stimuli (2 letters $\times 4$ orientations) presented in a continuous stream of $250 \mathrm{~ms}$ per letter. This was an attention demanding task (Kastner et al., 1998). Hence, target presentation rate in the target grating detection task matched the letter target presentation rate at fixation (also once every 2 seconds on average).

\section{fMRI Analyses}

The imaging data were analyzed using BrainVoyager QX (Brain Innovation, Maastricht, The Netherlands). Anatomical images were Talairach transformed (Talairach \& Tournoux, 1988) with a voxel size of $1 \mathrm{~mm}^{3}$. Functional measurements were co-registered to pre-standardized 3D anatomical space and preprocessed. Of each functional run, the first two images were excluded from data analysis. Functional images then were Talairach transformed with a resampled isotopic voxel size of $2 \mathrm{~mm}^{3}$, and pre-processed using slice time correction, 3D motion correction (sinc interpolation) and temporal filtering (linear trend removal and a high-pass filter of 3 cycles per time course). The spatially standardized anatomical images for each hemisphere were segmented according to the grey and white matter boundaries, and subsequently transformed into a 3D surface representation (Kriegeskorte and Goebel, 2001; Goebel et al., 2006). The 3D cortical surfaces were then inflated and flattened to achieve a 2D representation of the cortical surface of each hemisphere. Time series analysis of localizer data was conducted using a general linear model (GLM) for fMRI (Worsley \& Friston, 1995), using one predictor (checkerboard stimulus versus fixation) which was convolved with a two-gamma function to account for the hemodynamic response delay. Retinotopic mapping (polar angle mapping) (Engel, Glover, \& Wandell, 1997; Linden, Kallenbach, Heinecke, Singer, \& Goebel, 1999; Sereno et al., 1995) was used to delineate borders between V1, V2, and V3, which were mapped on inflated cortical renderings of individual participants. Regions of interest in V1, V2 and V3 for two quadrants of the visual field were determined using the retinotopic maps in combination with the localizer data. 

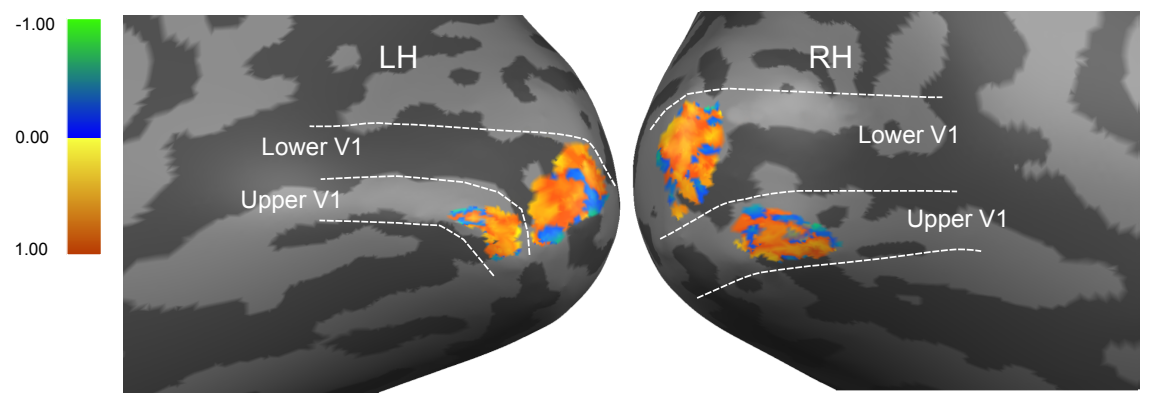

Figure 3. ROI and Voxel selection in a single participant. After determining a ROI from a localizer experiment, voxels were selected within the ROI based on their contrast sensitivity. Contrast sensitivity was determined for each voxel by fitting its responses to the range of contrasts we used $(1,2,3,4,6,8,16,32 \%)$ with a sigmoid function. Coloured patches depict voxels selected on the basis of localizer and retinotopic mapping. Colour scale depicts the goodness of fit based on the fitted sigmoid function; voxels were selected based on a goodness of fit criterion $\left(r^{2}>0.8\right)$.

\section{ROI and Voxel selection}

In order to optimize sensitivity of analyses, we first determined Regions of Interest (ROIs) $\left(5^{\circ} \varnothing, 5^{\circ}\right.$ eccentricity, two visual quadrants, visual area V1, V2 and V3) based on the localizer stimulus and the mapping of the retinotopic borders (see Methods). Then we made an extra subselection of contrast-sensitive voxels within each ROI. We selected voxels that showed contrast response functions, or put differently, increased BOLD response as a function of contrast. To do so we fitted a sigmoidal function to the contrast response of each voxel for each quadrant, in each visual area using 2 passive runs from the first fMRI session. Voxels were selected based on a goodness of fit criterion $\left(r^{2}>0.8\right)$, and Figure 3 shows an example of the voxel selection for a representative participant. The selection of voxels within a region of interest was defined by the intersection of the independent localizer results and the contrast response fitting procedure. Figure 4 shows the difference in average contrast response from all ROI voxels versus only the within-ROI subselection (single participant data from the trained quadrant in V1). The plots clearly show that our voxel selection procedure identified a subset of voxels showing a reliable contrast response function, while the clear contrast-dependency of the fMRI response is less clear when taking all ROI voxels into account. 

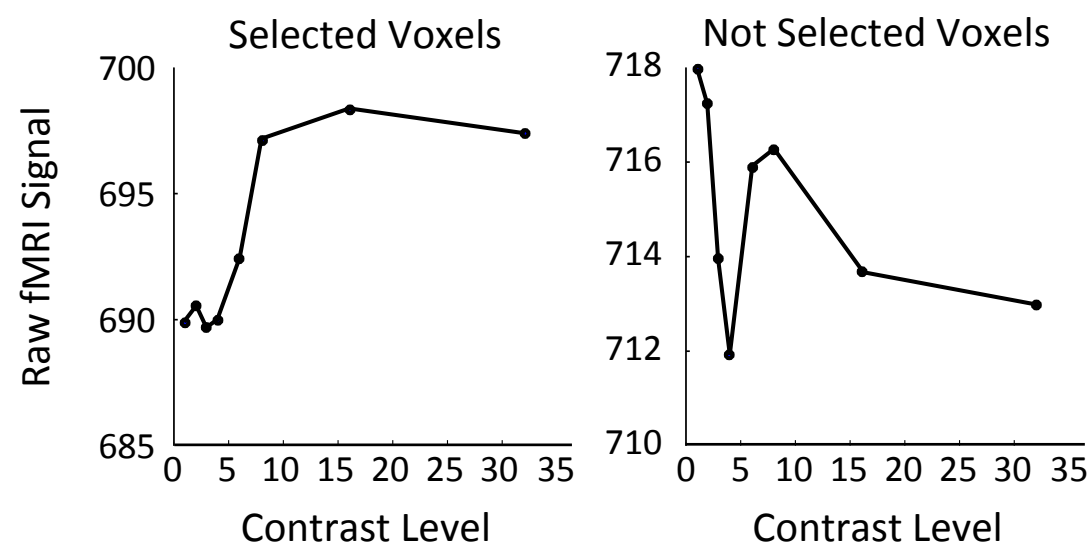

Figure 4. The result of Voxel selection. Left panel shows averaged contrast response function after voxel selection. Right panel shows averaged contrast response function without voxel selection. The left panel clearly shows a contrast response curve while the right panel does not.

\section{Results}

\section{Behavioural results}

Participants showed significant contrast learning during on average 6.7 training sessions in the psychophysics laboratory (Fig. 5). Contrast thresholds decreased from an average of $18 \%$ at the start of learning to $3.5 \%$ Michelson's contrast during asymptotic learning (session 1 versus $6, t=7.490, d f=6, p=0.0003$, paired T-test). Note that participants had also been trained in an otherwise unused lower visual quadrant (pre-training, see Task in Psychophysics laboratory). In Figure 6, we show that learning curves between the pre-test quadrant and the trained quadrant are very similar, suggesting that there is no generalisation between quadrants (Bonferroni corrected paired t-test, 6 sessions, $\mathrm{df}=$ $6, p>0.05)$. In the fMRI scanner, stimuli were presented using the method of constant stimuli (see Stimuli and experimental design in the fMRI scanner). To asses behavioural performance in the scanner we performed a receiver operating characteristic (ROC) analysis on the Active runs, and made a comparison between pre and post training performance. Figure 7 shows the results of that analysis, in which we calculated d' per contrast for the first and last fMRI session. We found that $d$ ' increased significantly for contrast 3\% $(t=-4.5971$, $\mathrm{df}=6, \mathrm{p}=0.0037$, Bonferroni corrected) and for contrast 32\% $(\mathrm{t}=-5.5009$, $\mathrm{df}=6, \mathrm{p}=0.0015$, Bonferroni corrected). The d' level for low contrasts in the first fMRI session was around zero, indicating that participants were not able to perform the task at low contrast levels before any training had taken 


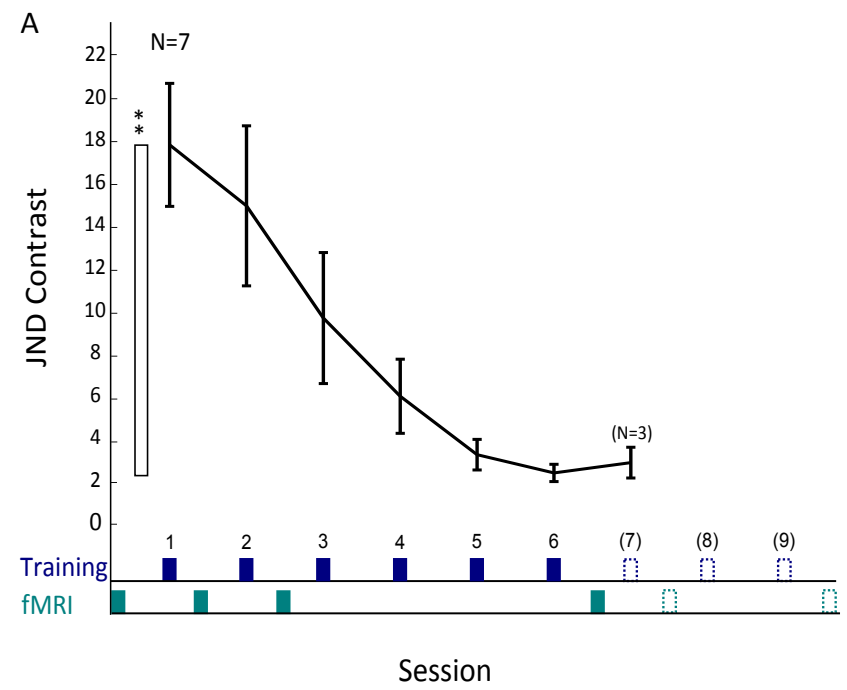

Figure 5. Behavioural training effects. (A) Learning curve resulting from psychophysical training sessions, the just noticeable difference (JND) plotted as a function of session. The grey bars on the $x$-axes depict the timing of the fMRI sessions, blue bars depict psychophysical training sessions (some participants trained and were scanned in extra sessions, dashed squares) depending on when they reached asymptotic performance. Contrast thresholds stabilized around $3 \%$ (error bars depict \pm one standard error). Asterisks indicate significant decreases in contrast thresholds when comparing session 1 to session $6(\mathrm{t}=7.490, \mathrm{df}=6, \mathrm{p}=0.0003$, paired T-test).

place. Note that d' was also close to zero during pre-training performance at the highest contrasts (see Discussion).

\section{FMRI data}

\section{Pre-Post comparison}

Voxel selection led on average to $67 \%$ (or 409 ) selected voxels, the amount and percentages of selected voxels were very similar across subjects, experimental quadrants and visual areas. ROl's for the two quadrants (trained, control, for V1, V2 and V3) were based upon selected voxels that showed a contrast response function with $80 \%$ goodness of fit (for details, see Methods). For both quadrants we compared the magnitude of the BOLD response (percent signal change) for the 8 different contrast levels for the passive runs between the first and last fMRI session. Figure 8 shows the resulting plots. For the control quadrant the only contrast that differed significantly between the pre and post training $\mathrm{fMRI}$ sessions, was the lowest contrast. For the trained quadrant, contrast 3 showed a significant difference in percent signal change 


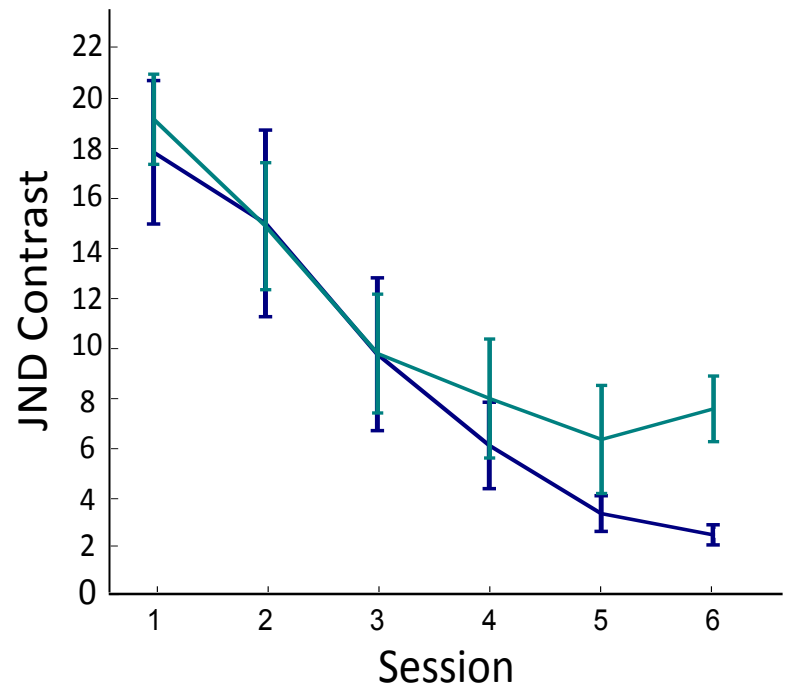

Figure 6. No transfer of learning between the two tested visual quadrants. The figure shows two learning curves, one that was determined before the start of the study in a further unused lower visual quadrant (green line) to determine the course of learning. The blue line depicts the contrast learning curve in the trained quadrant that was used for the main experiment (error bars depict \pm one standard error). The learning curves almost overlap indicating that pre-training performance did not transfer to the experimental, trained quadrant (Bonferroni corrected paired t-test, 6 sessions, $d f=$ $6, p>0.05)$. 

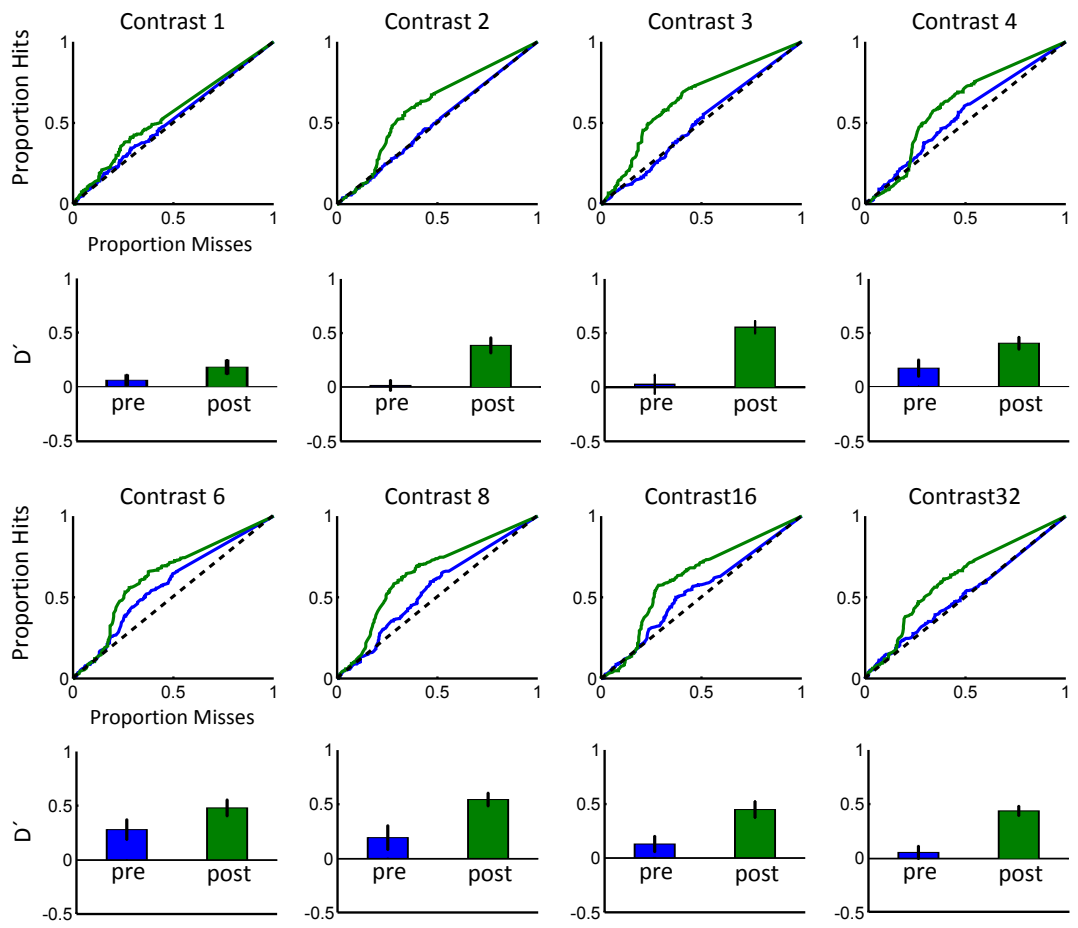

Figure 7. ROC analyses of participant's orientation discrimination performance for the 8 contrast levels, during the $\mathrm{fMRI}$ training sessions. Per contrast we plotted hits (correct positives) on the $y$-axes and misses (false negatives) on the $x$-axes. Hits and misses were determined as a function of reaction time criterion, between 0 and 1500 ms post stimulus, to determine ROC curves for behavioural data collected during the pre-training (blue line) and post-training (green line) fMRI sessions. The dotted line shows performance at chance level (HITS equal to misses). D' is defined as the z-values for hits minus the z-values for misses (Ehrenstein and Ehrenstein, 1999). Bar graphs show d' per contrast for both the pre-training (blue bars) and post-training fMRI training sessions (error bars depict \pm one standard error). 
of the BOLD response after learning $(t=2.0124$, $d f=6, p=0.045429$, not Bonferroni corrected). There were no significant differences between pre and post fMRI measures, for either quadrant in V2 and V3.

The results from the analysis above did not lend themselves for a longitudinal analysis as a function of learning/time, given the small effect. We made similar comparisons between first and second, first and third fMRI sessions etc., but no significant differences between sessions were found for these comparisons.

\section{Discussion}

Training on a grating contrast detection task at a fixed orientation difference significantly improved participants' performance. This was evident in the contrast thresholds obtained in the psychophysics laboratory, which showed a significant decrease from $18 \%$ to 3\% Michelson contrast (Fig. 5). Learning was fast between sessions 1-4, and was followed by slower asymptotic learning during session 5-7 (Karni \& Sagi, 1993) (Fig 5). In addition, we found that the decreased contrast thresholds at the end of learning in the pre-trained quadrant did not transfer to the trained quadrant used in the fMRI experiment, as learning curves were approximately the same for both quadrants (Fig. 6). Thus, contrast learning shows high position specificity, in line with a possible contribution of retinotopic areas to the learning of this skill.

Our results and analysis of the behavioural data and of the plasticity underlying memory formation in the task we employed have focused on a pre-training versus post-training comparison. The pre-training versus post-training comparison of the behavioural data collected during active runs in the scanner was carried out using ROC. The pre-training d'values varied across contrast levels in an unexpected manner. D' was close to zero for the 3 lowest contrasts $(1,2,3 \%)$, and then increased for $4 \%$ and $6 \%$ Michelson contrast, but then decreases again for the 3 highest contrasts $(8,16,32 \%$ ) (see Fig. 7 ). The pattern of the participants' pre-training behavioural data may be explained by participants' response strategies. For low contrasts, participants showed a large number of misses, or performed at chance level, which resulted in a d' close to zero. For medium contrasts participants seem to have opted for a conservative response strategy resulting in a high hit rate and almost no false positives leading to a higher d'. However when participants were presented with high contrast stimuli they appear to have adopted a very liberal strategy, resulting in many more hits but also more false positives resulting in a return to $d^{\prime}$ 'values close to zero. The post-training d' values showed a significant increase in d' compared to the pretraining d'values for 3\% Michelson's contrast (see Fig. 7). Prior to training, the orientation difference in the stimulus at that contrast was indistinguishable likely because the stimulus was undetectable ( $d$ ' close to zero). After training, $d^{\prime}$ at that contrast showed a significant increase, indicating that participants were able to perform the orientation detection above chance level. Note that we also found a significant difference between pre-training and post-training d' 

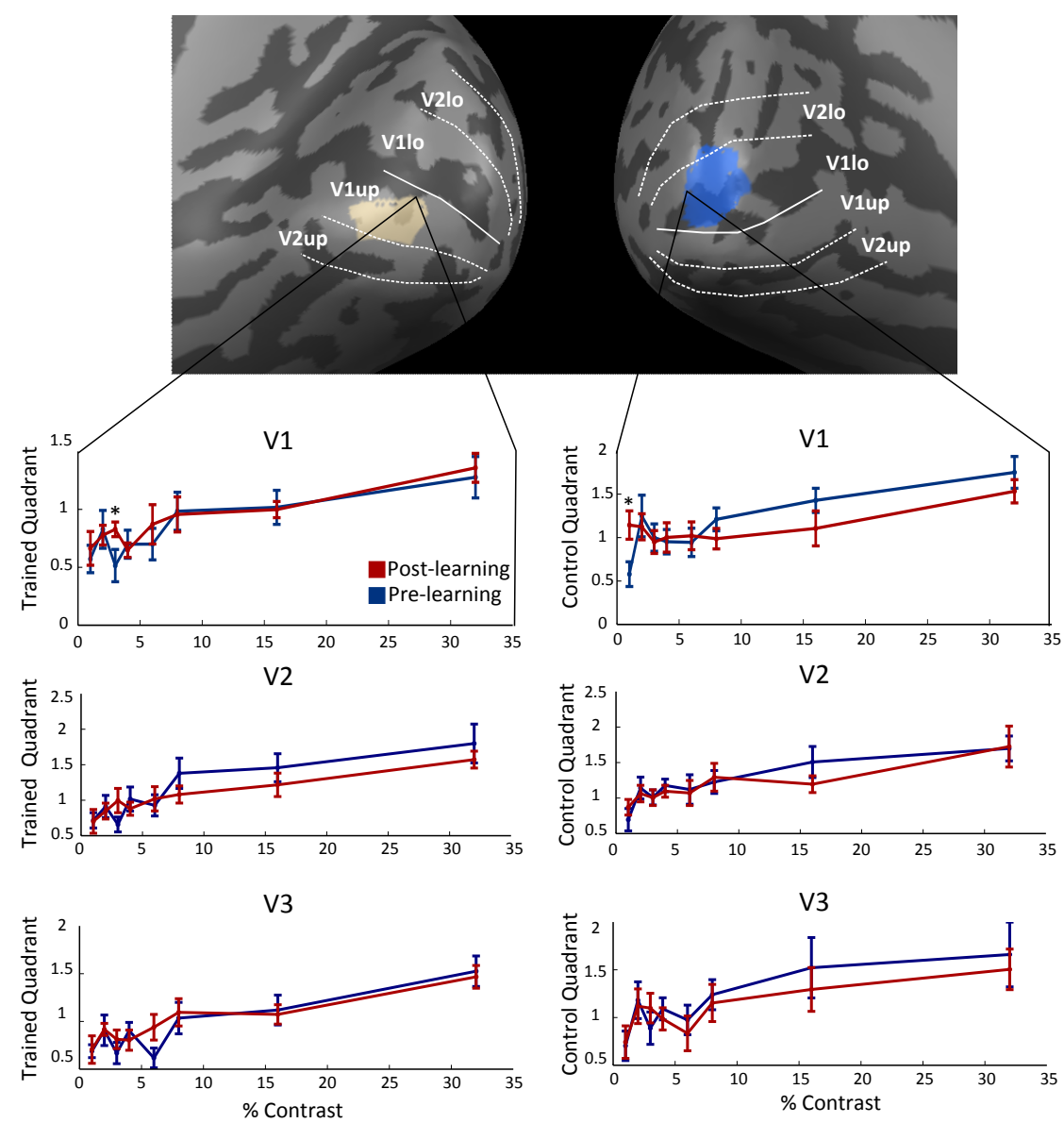

Figure 8. FMRI Results for V1 to V3 (from top to bottom) separated for the trained (left) and the control (right) quadrant. Here, the size of the BOLD response (percent signal change) is plotted as a function of the 8 different contrast levels, with the blue line depicting the first session and red line depicting the last fMRI session. In V1 for the control quadrant (upper right plot) the lines diverge significantly for the lowest contrast $(1 \%),(t=2.6404, d f=6, p=0.038$, not Bonferroni corrected), but not for any of the other contrast levels. For the trained quadrant in V1 (upper left plot), the contrast that shows a significant increase in BOLD response after learning is $3 \%$, $(\mathrm{t}=2.0124, \mathrm{df}=6, \mathrm{p}=0.045$, not Bonferroni corrected). Neither quadrant in V2 and $\mathrm{V} 3$ showed a modification of the contrast response function after learning (error bars depict \pm one standard error). 
at $32 \%$ contrast, which is related to the peculiarly low pre-training d'values at high contrasts.

The pre-training versus post-training comparison of the fMRI data has focused on the passive runs, to separate effects of plasticity in the fMRI signal from attention effects. We had hypothesized that skill learning would lead to an automatic incorporation of the attentional effect in the response to trained stimuli, and that the response to trained stimuli would resemble input gain (Reynolds et al., 2000). This would be reflected in a leftward shift of the contrast curve after learning (towards lower contrasts) compared to the contrast curve prior to learning. In order to investigate this we constructed a contrast response function from the $\mathrm{fMRI}$ data before and after training. In the trained quadrant, we expected a learning-dependent signal enhancement for the lower to middle contrasts, resembling a leftward shift of the contrast response function. This leftward shift was expected to be absent in the control quadrant where stimuli were only presented passively. The results of our analysis so far do not support a leftward shift of the contrast response function induced by training. It is possible that this effect was missed due to insufficient sensitivity of the fMRI signal and insufficient statistical power. Therefore, our data at present are inconclusive in their support of the hypothesis of visual skill as a form of 'frozen attention'. However, the small trend in V1 for elevated activity at lower contrasts after training points towards a possible contribution of V1 to contrast learning, in line with other imaging studies that have found increased activity after perceptual learning in human primary cortex (Vaina et al., 1998; Schwartz et al., 2002; Furmanski et al., 2004).

One of the main purposes of this study and its design was to measure the hypothesized changes in contribution of cortical areas along the visual hierarchy along the time course of learning, for which RHT and LLT make different predictions (see Introduction). To that goal, we scanned participants multiple times (on average 4.7 during training, at specific points during the course of learning (Fig 1 ). However, our pre-post comparison of fMRI effects revealed only small effects of learning. We made similar comparisons between the first and all intermediate $\mathrm{fMRI}$ sessions, but no significant differences between sessions were found for these comparisons. To our knowledge, the only other fMRI study that investigated human visual perceptual learning longitudinally is the study of Yotsumoto et al (2008). They found that together with the increase of task performance, V1 activation during the first week of learning also increased. However, despite maintained performance levels, during the second stage of learning the signal in V1 decreased. They concluded that the time course of learning-induced plasticity in visual tasks is uncoupled from the changes in behavioural performance. This conclusion should be interpreted cautiously, because during the fMRI sessions stimuli were attended. Therefore the effects that are reported in Yotsumoto et al. (2008) can be explained by the withdrawal of attention towards the end of learning, as increased skill is usually considered to be paired with increased automaticity of performance. 
The fMRI effects we have found are smaller than those reported in Furmanski et al. (2004), which measured contrast thresholds before and after training in a grating contrast detection task. It is possible that the small training-induced fMRI effect in our study is due to aspects of the chosen task or paradigm. In particular, Furmanski et al. (2004) trained participants in a pure detection task (stimulus present or not), whereas in our task, there was a double requirement of optimizing orientation discrimination at contrasts as low as possible. We had chosen this task as pilot experiments showed that a pure contrast detection task led to learning that was too fast for a longitudinal approach of the learning. The chosen orientation difference was kept supra-threshold to maximize contrast-related learning, rather than orientation-related learning. It is possible, however, that the chosen orientation differences were small enough to put demands on discrimination abilities in addition to contrast detection abilities. It is possible that both our fMRI and behavioural data reflect an interaction between the possibly opposing demands of enhancing detection and discrimination (Recanzone et al., 1990; Pinaud et al., 2006).

A number of unexplored analyses exist that still can be performed with respect to the longitudinal analysis of these data, and it is possible that these analyses will reveal stronger effects. One interesting analysis would pool data based on a participant's individual performance per session. This would increase statistical power, and decrease the problem of participant variation with respect to the speed and amount of learning at an absolute time point (session number) in the learning curve. In addition, an individual 'behavioural performance index' for a given session could be paired with an fMRI 'plasticity index' based on a comparison of fMRI activity from trained and control quadrants for that session. This would permit correlating fMRI plasticity indexes and behavioural performance indexes for all contrasts individually, or pooled. Any correlation present in the trained quadrant would be expected to be greater than the correlation based on fMRI data from the control quadrant. Finally, we believe that the study we have performed here at $3 \mathrm{~T}$ would have had a better chance for success at $7 \mathrm{~T}$, thanks to the increased signal to noise ratio and spatial resolution afforded by higher field strengths.

\section{References}

Ahissar M, Hochstein S (1996) Learning pop-out detection: specificities to stimulus characteristics. Vision Res 36:3487-3500.

Ahissar M, Hochstein S (2002) View from the top: hierarchies and reverse hierarchies in the visual system. Neuron 36:791-804.

Ahissar M, Hochstein S (2004) The reverse hierarchy theory of visual perceptual learning.

Trends Cogn Sci 8:457-464.

Ahissar M, Nahum M, Nelken I, Hochstein S (2009) Reverse hierarchies and sensory

learning. Philos Trans R Soc Lond B Biol Sci 364:285-299.

Appelle S (1972) Perception and discrimination as a function of stimulus 


\section{References}

orientation-Oblique effect in man and animals. Psychol Bull 78:266-278.

Ball K, Sekuler R (1987) Direction-specific improvement in motion discrimination. Vision Res 27:953-965.

Dinse HR, Kleibel N, Kalisch T, Ragert P, Wilimzig C, Tegenthoff M (2006) Tactile coactivation resets age-related decline of human tactile discrimination. Ann Neurol 60:88-94.

Ehrenstein WH, Ehrenstein A (1999) Psychophysical methods. New York: Springer. Fahle M, Poggio T (2002) Perceptual Learning: MIT Press.

Frenkel MY, Sawtell NB, Diogo AC, Yoon B, Neve RL, Bear MF (2006) Instructive effect of visual experience in mouse visual cortex. Neuron 51:339-349.

Furmanski CS, Schluppeck D, Engel SA (2004) Learning strengthens the response of primary visual cortex to simple patterns. Curr Biol 14:573-578.

Gilbert CD, Sigman M, Crist RE (2001) The neural basis of perceptual learning. Neuron 31:681-697.

Godde B, Stauffenberg B, Spengler F, Dinse HR (2000) Tactile coactivation-induced changes in spatial discrimination performance. J Neurosci 20:1597-1604.

Karni A, Sagi D (1991) Where practice makes perfect in texture discrimination: evidence for primary visual cortex plasticity. Proc Natl Acad Sci U S A 88:4966-4970.

Karni A, Bertini G (1997) Learning perceptual skills: behavioral probes into adult cortical plasticity. Curr Opin Neurobiol 7:530-535.

Kastner S, De Weerd P, Desimone R, Ungerleider LG (1998) Mechanisms of directed attention in the human extrastriate cortex as revealed by functional MRI. Science 282:108-111.

Law CT, Gold JI (2008) Neural correlates of perceptual learning in a sensory-motor, but not a sensory, cortical area. Nat Neurosci 11:505-513.

Pinaud R, Tremere L, De Weerd P, eds (2006) Plasticity in the visual system: From genes to circuits. New York: Springer.

Raiguel S, Vogels R, Mysore SG, Orban GA (2006) Learning to see the difference specifically alters the most informative V4 neurons. J Neurosci 26:6589-6602.

Recanzone GH, Allard TT, Jenkins WM, Merzenich MM (1990) Receptive-field changes induced by peripheral nerve stimulation in SI of adult cats. J Neurophysiol 63:1213-1225. Reynolds JH, Pasternak T, Desimone R (2000) Attention increases sensitivity of V4 neurons. Neuron 26:703-714.

Schoups A, Vogels R, Qian N, Orban G (2001) Practising orientation identification improves orientation coding in V1 neurons. Nature 412:549-553.

Schoups AA, Vogels R, Orban GA (1995) Human perceptual learning in identifying the oblique orientation: retinotopy, orientation specificity and monocularity. J Physiol 483 ( Pt 3):797-810.

Schwartz S, Maquet P, Frith C (2002) Neural correlates of perceptual learning: a functional MRI study of visual texture discrimination. Proc Natl Acad Sci U S A 99:17137-17142. Vaina LM, Belliveau JW, des Roziers EB, Zeffiro TA (1998) Neural systems underlying learning and representation of global motion. Proc Natl Acad Sci U S A 95:12657-12662. Watanabe T, Nanez JE, Sasaki Y (2001) Perceptual learning without perception. Nature 413:844-848. 
5 Neural correlates of contrast learning in the human visual cortex

Wetherill GB, Levitt H (1965) Sequential Estimation of Points on a Psychometric Function. Br J Math Stat Psychol 18:1-10.

Xiao LQ, Zhang JY, Wang R, Klein SA, Levi DM, Yu C (2008) Complete transfer of perceptual learning across retinal locations enabled by double training. Curr Biol 18:1922-1926.

Yang T, Maunsell JH (2004) The effect of perceptual learning on neuronal responses in monkey visual area V4. J Neurosci 24:1617-1626.

Yotsumoto Y, Watanabe T, Sasaki Y (2008) Different dynamics of performance and brain activation in the time course of perceptual learning. Neuron 57:827-833.

Zhang J, Kourtzi Z (2010) Learning-dependent plasticity with and without training in the human brain. Proc Natl Acad Sci U S A 107:13503-13508. 
6 Discussion and summary 


\section{General Discussion}

The neural changes that are induced by learning or exposure to stimuli are generally referred to as plasticity. The extent of adult plasticity in early sensory areas, however, is still a matter of debate, as well as the involved mechanisms. The present thesis contributes to this debate on four specific topics, by investigating (1) the presence of learning-induced changes in primary sensory cortex, (2) the time course of learning-induced plasticity at multiple levels of sensory systems to test Reversed Hierarchy Theory (RHT) (Ahissar and Hochstein, 2002, 2004; Ahissar et al., 2009) and Lowest Level Theories (LLT) (Karni and Bertini, 1997), (3) the link between attention and visual skill learning and (4) the evidence underlying the concept of consolidation.

Specificity of learning for retinal location and stimulus feature has been interpreted as evidence for the involvement of early visual areas during learning and plasticity. In the literature there is evidence for retinal location specificity in studies of orientation discrimination (Schoups et al., 1995), for motion discrimination (Ball and Sekuler, 1987) and texture discrimination (Karni and Sagi, 1991; Ahissar and Hochstein, 1996). In addition to specificity for position, perceptual learning is also specific for the orientation of the stimulus or stimulus elements (Ramachandran and Braddick, 1973; McKee and Westheimer, 1978; Fiorentini and Berardi, 1980, 1981; Ball and Sekuler, 1987; Karni and Sagi, 1991; Poggio et al., 1992; Fahle and Edelman, 1993; Schoups et al., 1995; Ahissar and Hochstein, 1996; Westheimer et al., 2001). However a recent report from Xiao et al. (2008) demonstrated complete transfer of training from one to another location in a so-called 'double training paradigm'. Participants were trained on a feature (contrast) at location one and then additional training of an irrelevant feature (orientation) took place at a second location in the visual field. This additional training caused complete transfer of feature learning (i.e. contrast) to the second location. It is important to note that subjects were trained during 6 sessions, and it might be the case that asymptotic learning was not reached yet and that specificity was therefore missed. Nevertheless, Xiao et al. (2008) have reopened the debate on specificity of visual perceptual learning, and thereby the presence of plasticity in early visual cortex. In the research presented here, there are four findings that support the specificity of perceptual learning and the presence of plasticity in low-level visual cortex. First, in chapter 2 we demonstrated behavioural interference by training two orientation discrimination tasks consecutively in the same quadrant. Interference was measured against performance in a control quadrant, where participants performed only a single orientation discrimination task. The pattern of results, with interference confined to a single quadrant, implies position specificity of the plastic processes underlying memory formation induced by training a visual perceptual skill (orientation discrimination). Moreover, we found that the extent of interference depended on the difference in orientation between the reference orientations of the consecutively performed tasks. This 
indicates that processes forming the basis of visual perceptual skill also show orientation specificity. The position and the orientation specificity of interference thus support the idea of plastic changes in low-level visual cortex underlying the emergence of visual skills. Second, in chapter 3, we showed interference in a visual learning paradigm using TMS. This interference was conditional on task context and occurred only when training in the location targeted by TMS was followed by training in a second location before TMS was applied. Importantly, the TMS effect did not generalize across quadrants. These findings support the hypothesis of location-specific mechanisms of plasticity, likely at early sites of the visual hierarchy. Third, in chapter 5, we collected contrast thresholds as a measure of performance in an orientation discrimination task at a constant orientation difference. Learning curves were obtained sequentially in two different quadrants. We found no evidence for any generalization, as contrast thresholds at the start of training in the second visual quadrant were not different from thresholds at the start of the preceding training in the first quadrant. This indicates that the training effects were very location specific thereby supporting plasticity in early visual cortex as a mechanism for visual skill learning. Fourth, our fMRI data in Chapters 4 and 5 also provide support for plasticity in early visual cortex. We found increased fMRI activity in V1 during passive presentation of stimuli at contrasts for which behavioural performance had increased most after learning. These results are in line with recent imaging studies that show increased V1 activity after long-term learning (Vaina et al., 1998; Schwartz et al., 2002; Furmanski et al., 2004). Moreover, by employing MVPA, we found a significant increase in classification accuracy in V1 at the contrasts matching those for which we had observed significant training-induced enhancements. Interestingly, these observations were done nine weeks after the conclusion of contrast learning, suggesting that V1 plays a role in the long-term maintenance of visual skills (if there is no interference). Taken together, our data indicate a significant contribution of V1 to the formation and long-term maintenance of visual skill memory.

The second topic to which this thesis contributes is the question whether plastic changes related to visual skill learning with simple stimuli are limited to low-level areas at the end of learning (LLT) or whether they involve the entire visual hierarchy (RHT). RHT and read-out theories in general predict that plasticity starts early in training in high-level areas of the sensory system and trickles down to lower-level areas towards the end of learning. This 'trickle-down' process permits high-level areas to access increasingly specific information in increasingly lower areas as required by the increased processing requirements developing in the course of learning. LLT, in contrast, predicts rewiring within low-level visual areas to increase the accuracy of stimulus processing when the requirements of the task exceed available processing capabilities. As this increased processing demand occurs during the asymptotic phase of learning, plasticity in low-level areas is expected only towards completion of learning. Hence, both LLT and RHT predict plasticity in low-level visual areas at the 


\section{Discussion}

end of learning, and this effect, if present, therefore cannot be used to distinguish the two theories. However, RHT predicts plasticity in mid to high-level visual areas in relatively early phases of learning, whereas these effects are not expected in LLT. Therefore, in Chapter 5, we aimed to track activity changes related to plasticity in multiple visual areas at the same time, to test these two categories of theories. The index of learning we aimed to use was a traininginduced, enhanced response to passively shown low contrast gratings (without attention, see Chapter 5). However, this index revealed only very weak effects when comparing the pre- and post-training fMRI sessions, and we did not find significant differences induced by training when comparing the pre-training session with any of the intermediate sessions. This negative conclusion held in $\mathrm{V} 1$, V2, and V3. Hence, our analysis on the time course of plasticity in multiple visual areas so far did not reveal any effects that could be used to distinguish LLT from RHT. However, in chapter 3, the finding that TMS directed to V1 interfered with orientation discrimination learning from the first session is highly relevant for this debate. Our findings indicate that low-level visual areas are being accessed for readout from the very beginning of learning. This is difficult to reconcile with $\mathrm{RHT}$, as it suggests that information is read out from V1 from the beginning of learning. This is an interesting finding which requires revision of both RHT and LLT type theories, as it suggests training-induced plasticity in a network of areas that is much broader than anticipated at time point in the learning curve when neither theory would have predicted it.

Both the RHT and LLT assume a critical contribution of attention to perceptual learning. Reynolds and colleagues (2000) proposed that the main function of attention is to enhance the processing of weak stimuli, and found a leftward shift in the contrast response function as a result of attention leading them to propose an input gain model where attention selectively enhances weak inputs. Several studies have confirmed that visual perceptual learning indeed only occurs for those stimulus features that are attended (task relevant features) (Shiu and Pashler, 1992; Ahissar et al., 2001; Schoups et al., 2001), although it is now known that there are forms of perceptual learning that can take place outside the focus of attention, or even unconsciously (Watanabe et al., 2001; Watanabe et al., 2002; Seitz and Watanabe, 2003). Moreover, also pure exposure (Frenkel et al., 2006; Gutnisky et al., 2009) and reward signals (Seitz et al., 2009; Franko et al., 2010) can trigger perceptual learning. In chapter 5 we studied the contribution of attention to visual perceptual learning by training subjects on a range of contrasts and assed the effects of learning using fMRI. In the scanner, participants were exposed (in a subset of runs) to grating stimuli at a range of contrasts, while they performed an attention-demanding task at fixation. In this way, we measured an fMRI response contrast function before and after training. We had expected that the contrast curve after learning would have shifted leftwards (towards lower contrasts) compared to the contrast curve prior to learning. Our hypothesis was that skill learning would lead to an automatic incorporation of the attentional effect in the response to trained stimuli, 
and that the response to trained stimuli would resemble input gain. Our results did show significant enhancement of a low to medium contrast after learning $(3 \%)$, however we did not find a leftward shift of the contrast response function. It is likely that this effect was missed due to insufficient sensitivity of the fMRI signal and insufficient statistical power. Therefore, our data at present are too weak to support the hypothesis of visual skill as a form of 'frozen attention'.

Following learning, plasticity takes place for a discrete period of time after completion of the learning experience. During this time period, memory traces are thought to be malleable and susceptible to interference (Dudai, 2004). According to classical views (Dudai, 2004), memory traces become 'consolidated' upon closure of a several hour long period of plasticity, hence the concept of 'time-limited consolidation'. Several studies have found evidence of behavioural interference for time intervals of up to a few hours between consecutive tasks in support of time-limited consolidation in the motor domain (Brashers-Krug et al., 1996; Shadmehr and Holcomb, 1997) and visual domain (Seitz et al., 2005). However, other studies have shown interference for very long time intervals (Goedert and Willingham, 2002; Caithness et al., 2004), raising doubts about the concept of time-limited consolidation. In chapter 2 we studied behavioural interference of two visual skill learning tasks during asymptotic learning. We found behavioural interference of which the magnitude was independent of the time interval between consecutive tasks (for intervals up to $24 \mathrm{~h}$ ), and moreover, we found that the interference depended on the orientation difference between references used in consecutive tasks. This indicates that the amount of overlap between orientation selective neuronal populations involved in the two tasks was the determining factor for behavioural interference. The finding of interference when tasks are performed that recruit overlapping neuronal populations can be interpreted in two ways. It could suggest a long consolidation period, exceeding $24 \mathrm{~h}$, in which case the finding of behavioural interference would truly be an indication of malleability of the memory trace during the consolidation. Alternatively, the behavioural interference we observed may indicate that even after closure of learning-induced plasticity, memory traces might remain malleable, and never fully consolidate. This idea is in line with the multiple trace theory proposed in the domain of episodic memory formation (Lewis, 1979; Nadel and Moscovitch, 2001). According to this theory memories when retrieved will be susceptible to reconsolidation (Lee et al., 2004; Lee, 2008). A similar view proposes memory traces to be active when they are formed, to become inactive when not accessed and to be reactivated when retrieved ( $\mathrm{Xu}$ et al., 2009; Yang et al., 2009). Hence, during retrieval memory traces would return to a malleable state during which they can be modified. In the context of visual skill learning in subsequent daily sessions, it would be more efficient to modify the memory trace laid down in the preceding session than creating separate memory traces for each learning experience. In this sense, our data are most parsimoniously interpreted in the theoretical context of memory trace reactivation/reconsolidation. 


\section{Discussion}

The interference observed in chapter 2 emerged only after several training sessions on the condition that the orientation selective neurons recruited in the two trained tasks were overlapping. The delay in the emergence of interference indicates that orientation selective mechanisms started to contribute to learning only after several sessions of training. Hence, our behavioural interference design revealed contributions of mechanisms in lower-order visual areas. Interestingly, the TMS-induced interference observed in Chapter 3 appeared after the very first learning session. This indicates that the mechanism underlying interference with TMS is different from the mechanism underlying interference induced by consecutive performance of two tasks recruiting overlapping neuronal populations. The TMS-induced interference was context-dependent, and therefore more in line with high-level processes that are thought to play a role in setting up task routines at the outset of perceptual learning. The TMS data thus indicate that $\mathrm{V} 1$ is involved in these high-level processes, in addition to more stimulus specific plastic processes occurring later on in the learning curve.

In summary, the behavioural, TMS and fMRI research presented here has demonstrated evidence for the contribution of human area V1 to the formation of training-induced visual skill memories. Psychophysical evidence indicates that the plasticity mechanisms involved in memory formation lead to memory traces that remain highly malleable, rather than fully consolidated. The use of TMS revealed a contribution of V1 to plastic processes following the end of the very first training session, which requires modification of influential theories of perceptual learning. In visual skill learning tasks that require enhanced sensitivity to contrast, we found highly position-specific learning, and we also found an enhanced V1 fMRI response to stimuli at a low contrast belonging to the range of contrasts showing the greatest behavioural performance enhancement. However, the effects were too weak to support the hypothesis of skill as a form of hard-wired attention. The latter effect (or the absence thereof) is instructive in evaluating the sensitivity of $\mathrm{fMRI}$ in tracking small changes in neural activity as an index of plasticity in the course of perceptual learning.

\section{Future perspectives}

To distinguish between RHT and LLT, we have aimed to assess the involvement of visual areas across the visual hierarchy, over the full time-course of visual perceptual learning. Although our current analysis so far did not allow us to test the time course of plasticity in multiple areas, additional analysis in the current dataset or new datasets should be considered to test RHT and LLT. Measures of effective connectivity (Goebel et al., 2003; Roebroeck et al., 2005, 2011) can shed light on the dynamic changes in recruitment of visual areas during the course of learning. From the perspective of RHT we would expect strongest effective connectivity patterns limited to the higher visual areas at the start of learning when read out routines are being set up. When learning progresses towards asymptotic performance, effective connectivity patterns between higher 
visual areas and primary visual area are expected to gain in strength. Thus, RHT would predict an expanding network that would include the lowest level areas only towards the end of learning. It would be interesting to test this prediction, especially since the TMS interference paradigm we used (Chapter 3) does not support this prediction. By contrast, LLT predicts local plasticity to happen in the lowest visual area that is necessary for the task, and that in the case of training in an orientation discrimination task any network changes are expected to remain limited to V1 (and possibly directly associated retinotopic areas).

To successfully test the involvement of visual areas during the time course of perceptual learning it would be beneficial to enhance the signal to noise ratio (SNR) of our measures, as learning dependant signal changes have proved to be of a small magnitude. The advent of (ultra) high field fMRI gives us the opportunity to optimize both SNR and spatial/temporal resolution to answer specific questions regarding the location of plasticity induced by visual perceptual learning. For example, using 3T, BOLD activation from voxels represent averaged activity form all cortical layers, while the layers of greatest interest for perceptual learning are the superficial layers (Yacoub et al., 2008; Bissig and Berkowitz, 2009; Koopmans et al., 2010). At high field strengths, the ability to resolve the activity patterns originating from different layers (Koopmans et al., 2010; Polimeni et al., 2010; Koopmans et al., 2011; Zimmermann et al., 2011; Olman et al., 2012) may unveil layer specific learning effects that would currently remain masked by the activity of the cortical layers showing less plasticity. In addition, high field strength may allow one to resolve activity at the level of orientation columns (Yacoub et al., 2008), which may open the possibility to explore voxel patterns at better spatial resolution (Formisano and Kriegeskorte, 2012). Animal models also provide a useful approach to longitudinally investigate visual skill learning. Of particular interest is the possibility to implant several depth probes (Schroeder et al., 1998; Yoon et al., 2000) or other recording devices, because they give the opportunity to monitor activity of a constant population of neurons during and after learning. Depth probes permit simultaneous recording of layer-specific activity across several retinotopic areas. An alternative technique is the sub- or supradural insertion of large-scale ECoG grids (Rubehn et al., 2009). This grid can be used to record ultra-high spatial resolution EEG across the surface of a hemisphere, or of the entire brain, which can be especially useful for tracking dynamic learning-dependent changes in functional connectivity among brain areas.

Neuroscience is inherently multidisciplinary, and advanced understanding will increasingly depend on the combination of insights from neurophysiological and molecular approaches in animals with measures of human brain activity. In this respect, the combination of work in non-human and human primates will be highly useful, especially when exploring the new opportunities offered by high-field and ultra-high-field fMRI. In this context, the present thesis hopefully can represent a small piece in the great puzzle of neuroscience. 


\section{Discussion}

\section{References}

Ahissar M, Hochstein S (1996) Learning pop-out detection: specificities to stimulus characteristics. Vision Res 36:3487-3500.

Ahissar M, Hochstein S (2002) View from the top: hierarchies and reverse hierarchies in the visual system. Neuron 36:791-804.

Ahissar M, Hochstein S (2004) The reverse hierarchy theory of visual perceptual learning. Trends Cogn Sci 8:457-464.

Ahissar M, Laiwand R, Hochstein S (2001) Attentional demands following perceptual skill training. Psychol Sci 12:56-62.

Ahissar M, Nahum M, Nelken I, Hochstein S (2009) Reverse hierarchies and sensory learning. Philos Trans R Soc Lond B Biol Sci 364:285-299.

Ball K, Sekuler R (1987) Direction-specific improvement in motion discrimination. Vision Res 27:953-965.

Bissig D, Berkowitz BA (2009) Manganese-enhanced MRI of layer-specific activity in the visual cortex from awake and free-moving rats. Neuroimage 44:627-635.

Brashers-Krug T, Shadmehr R, Bizzi E (1996) Consolidation in human motor memory.

Nature 382:252-255.

Caithness G, Osu R, Bays P, Chase H, Klassen J, Kawato M, Wolpert DM, Flanagan JR (2004) Failure to consolidate the consolidation theory of learning for sensorimotor adaptation tasks. J Neurosci 24:8662-8671.

Dudai Y (2004) The neurobiology of consolidations, or, how stable is the engram? Annu Rev Psychol 55:51-86.

Fahle M, Edelman S (1993) Long-term learning in vernier acuity: effects of stimulus orientation, range and of feedback. Vision Res 33:397-412.

Fiorentini A, Berardi N (1980) Perceptual learning specific for orientation and spatial frequency. Nature 287:43-44.

Fiorentini A, Berardi N (1981) Learning in grating waveform discrimination: specificity for orientation and spatial frequency. Vision Res 21:1149-1158.

Formisano E, Kriegeskorte N (2012) Seeing patterns through the hemodynamic veil - The future of pattern-information fMRI. Neuroimage.

Franko E, Seitz AR, Vogels R (2010) Dissociable neural effects of long-term stimulus-reward pairing in macaque visual cortex. J Cogn Neurosci 22:1425-1439.

Frenkel MY, Sawtell NB, Diogo AC, Yoon B, Neve RL, Bear MF (2006) Instructive effect of visual experience in mouse visual cortex. Neuron 51:339-349.

Furmanski CS, Schluppeck D, Engel SA (2004) Learning strengthens the response of primary visual cortex to simple patterns. Curr Biol 14:573-578.

Goebel R, Roebroeck A, Kim DS, Formisano E (2003) Investigating directed cortical interactions in time-resolved fMRI data using vector autoregressive modeling and Granger causality mapping. Magn Reson Imaging 21:1251-1261.

Goedert KM, Willingham DB (2002) Patterns of interference in sequence learning and prism adaptation inconsistent with the consolidation hypothesis. Learn Mem 9:279-292.

Gutnisky DA, Hansen BJ, lliescu BF, Dragoi V (2009) Attention alters visual plasticity during exposure-based learning. Curr Biol 19:555-560. 
Karni A, Sagi D (1991) Where practice makes perfect in texture discrimination: evidence for primary visual cortex plasticity. Proc Natl Acad Sci U S A 88:4966-4970.

Karni A, Bertini G (1997) Learning perceptual skills: behavioral probes into adult cortical plasticity. Curr Opin Neurobiol 7:530-535.

Koopmans PJ, Barth M, Norris DG (2010) Layer-specific BOLD activation in human V1. Hum Brain Mapp 31:1297-1304.

Koopmans PJ, Barth M, Orzada S, Norris DG (2011) Multi-echo fMRI of the cortical laminae in humans at $7 \mathrm{~T}$. Neuroimage 56:1276-1285.

Lee JL (2008) Memory reconsolidation mediates the strengthening of memories by additional learning. Nat Neurosci 11:1264-1266.

Lee JL, Everitt BJ, Thomas KL (2004) Independent cellular processes for hippocampal memory consolidation and reconsolidation. Science 304:839-843.

Lewis DJ (1979) Psychobiology of active and inactive memory. Psychol Bull 86:1054-1083. McKee SP, Westheimer G (1978) Improvement in vernier acuity with practice. Percept Psychophys 24:258-262.

Nadel L, Moscovitch M (2001) The hippocampal complex and long-term memory revisited. Trends Cogn Sci 5:228-230.

Olman CA, Harel N, Feinberg DA, He S, Zhang P, Ugurbil K, Yacoub E (2012)

Layer-specific FMRI reflects different neuronal computations at different depths in human v1. PLoS One 7:e32536.

Poggio T, Fahle M, Edelman S (1992) Fast perceptual learning in visual hyperacuity. Science 256:1018-1021.

Polimeni JR, Fischl B, Greve DN, Wald LL (2010) Laminar analysis of 7 T BOLD using an imposed spatial activation pattern in human V1. Neuroimage 52:1334-1346.

Ramachandran VS, Braddick O (1973) Orientation-specific learning in stereopsis.

Perception 2:371-376.

Reynolds JH, Pasternak T, Desimone R (2000) Attention increases sensitivity of V4 neurons. Neuron 26:703-714.

Roebroeck A, Formisano E, Goebel R (2005) Mapping directed influence over the brain using Granger causality and fMRI. Neuroimage 25:230-242.

Roebroeck A, Formisano E, Goebel R (2011) The identification of interacting networks in the brain using fMRI: Model selection, causality and deconvolution. Neuroimage 58:296-302. Rubehn B, Bosman C, Oostenveld R, Fries P, Stieglitz T (2009) A MEMS-based flexible multichannel ECoG-electrode array. J Neural Eng 6:036003.

Schoups A, Vogels R, Qian N, Orban G (2001) Practising orientation identification improves orientation coding in $\mathrm{V} 1$ neurons. Nature 412:549-553.

Schoups AA, Vogels R, Orban GA (1995) Human perceptual learning in identifying the oblique orientation: retinotopy, orientation specificity and monocularity. J Physiol 483 ( Pt 3):797-810.

Schroeder CE, Mehta AD, Givre SJ (1998) A spatiotemporal profile of visual system activation revealed by current source density analysis in the awake macaque. Cereb Cortex 8:575-592.

Schwartz S, Maquet P, Frith C (2002) Neural correlates of perceptual learning: a functional MRI study of visual texture discrimination. Proc Natl Acad Sci U S A 99:17137-17142. 


\section{Discussion}

Seitz AR, Watanabe T (2003) Psychophysics: Is subliminal learning really passive? Nature 422:36.

Seitz AR, Kim D, Watanabe T (2009) Rewards evoke learning of unconsciously processed visual stimuli in adult humans. Neuron 61:700-707.

Seitz AR, Yamagishi N, Werner B, Goda N, Kawato M, Watanabe T (2005) Task-specific disruption of perceptual learning. Proc Natl Acad Sci U S A 102:14895-14900.

Shadmehr R, Holcomb HH (1997) Neural correlates of motor memory consolidation.

Science 277:821-825.

Shiu LP, Pashler H (1992) Improvement in line orientation discrimination is retinally local but dependent on cognitive set. Percept Psychophys 52:582-588.

Vaina LM, Belliveau JW, des Roziers EB, Zeffiro TA (1998) Neural systems underlying learning and representation of global motion. Proc Natl Acad Sci U S A 95:12657-12662. Watanabe T, Nanez JE, Sasaki Y (2001) Perceptual learning without perception. Nature 413:844-848.

Watanabe T, Nanez JE, Sr., Koyama S, Mukai I, Liederman J, Sasaki Y (2002) Greater plasticity in lower-level than higher-level visual motion processing in a passive perceptual learning task. Nat Neurosci 5:1003-1009.

Westheimer G, Crist RE, Gorski L, Gilbert CD (2001) Configuration specificity in bisection acuity. Vision Res 41:1133-1138.

Xiao LQ, Zhang JY, Wang R, Klein SA, Levi DM, Yu C (2008) Complete transfer of perceptual learning across retinal locations enabled by double training. Curr Biol 18:1922-1926.

Xu T, Yu X, Perlik AJ, Tobin WF, Zweig JA, Tennant K, Jones T, Zuo Y (2009) Rapid formation and selective stabilization of synapses for enduring motor memories. Nature 462:915-919.

Yacoub E, Harel N, Ugurbil K (2008) High-field fMRI unveils orientation columns in humans. Proc Natl Acad Sci U S A 105:10607-10612.

Yang G, Pan F, Gan WB (2009) Stably maintained dendritic spines are associated with lifelong memories. Nature 462:920-924.

Yoon TH, Hwang EJ, Shin DY, Park SI, Oh SJ, Jung SC, Shin HC, Kim SJ (2000) A micromachined silicon depth probe for multichannel neural recording. IEEE Trans Biomed Eng 47:1082-1087.

Zimmermann J, Goebel R, De Martino F, van de Moortele PF, Feinberg D, Adriany G, Chaimow D, Shmuel A, Ugurbil K, Yacoub E (2011) Mapping the organization of axis of motion selective features in human area MT using high-field fMRI. PLoS One 6:e28716. 


\section{Summary}




\section{Summary}

The neural changes that are induced by learning or exposure to stimuli are generally referred to as plasticity. The extent of adult plasticity in early sensory areas, however, is still a matter of debate, as well as the involved mechanisms. The present thesis contributed to this debate on four specific topics, by investigating (1) the presence of learning-induced changes in primary sensory cortex, (2) the time course of learning-induced plasticity at multiple levels of sensory systems to test Reversed Hierarchy Theory (RHT) (Ahissar and Hochstein, 2002, 2004; Ahissar et al., 2009) and Lowest Level Theories (LLT) (Karni and Bertini, 1997), (3) the link between attention and visual skill learning and (4) the evidence underlying the concept of consolidation. The collected research presented here; behavioural, TMS and FMRI research, has demonstrated evidence for the contribution of human area V1 to the formation of training-induced visual skill memories. Psychophysical evidence indicates that the plasticity mechanisms involved in memory formation lead to memory traces that remain highly malleable, rather than fully consolidated. The use of TMS revealed a contribution of $\mathrm{V} 1$ to plastic processes following the end of the very first training session, which requires modification of influential theories of perceptual learning. In visual skill learning tasks that require enhanced sensitivity to contrast, we found highly position-specific learning, and we also found an enhanced V1 fMRI response to stimuli at a low contrast belonging to the range of contrasts showing the greatest behavioural performance enhancement. However, the effects were too weak to support the hypothesis of skill as a form of hard-wired attention.

\section{Samenvatting}

Plasticiteit kan gedefinieerd worden als de neurale veranderingen gerelateerd aan het leren en blootstelling aan stimuli. De betrokkenheid van de vroege sensorische hersengebieden in het volwassen brein bij plasticiteit, is nog altijd open voor debat, evenals de mogelijke onderliggende mechanismen. Het onderzoek in deze thesis heeft bijgedragen aan dit debat door te focussen op de volgende vier onderwerpen; (1) het aanwezig zijn van door leren geïnduceerde veranderingen in primaire sensorische cortex, (2) het tijdsverloop van door leren geïnduceerde plasticiteit, en de betrokkenheid van verschillende niveau's van sensorische systemen, om zodoende de voorspellingen van de 'Reversed Hierarchy Theory' (RHT) (Ahissar and Hochstein, 2002, 2004; Ahissar et al., 2009) en 'Lowest Level Theories' (LLT) (Karni and Bertini, 1997) te testen, (3) de link tussen aandacht en visueel leren en (4) het bewijs onderliggend aan het concept van consolidatie. Het verzamelde onderzoek dat in deze thesis wordt gepresenteerd omvat zowel gedragsonderzoek evenals TMS en fMRI onderzoek, en laat evidentie zien voor een bijdrage van V1 aan de formatie van door leren geïnduceerd visueel vaardigheidsgeheugen. Psychofysische experimenten wijzen 
uit dat de mechanismen van plasticiteit, die betrokken zijn bij geheugenformatie, geheugensporen teweeg brengen die hoogst veranderlijk blijven in plaats van ten volle geconsolideerd te worden. Het TMS experiment toonde een bijdrage van $\mathrm{V} 1$ aan plastische processen volgend op het einde van een allereerste trainingssessie. Dit vraagt om het herzien van zeer invloedrijke theorieën over perceptueel leren. In visuele vaardigheidstaken die een verhoogde gevoeligheid voor stimulus contrast vereisen, vonden we dat perceptueel leren zeer locatie specifiek is. Bovendien vonden we een verhoging in het $\mathrm{fMRI}$ signaal dat gelinkt wordt aan een laag contrast komend uit een reeks van stimuluscontrasten waarvoor proefpersonen de grootste vooruitgang in performantie laten zien. Helaas waren de effecten te klein om een onvoldongen bewijs te vormen voor de hypothese dat leren een vorm van 'hard-wiring' van aandacht is. 

8 Curriculum Vitae 


\section{Curriculum Vitae}

Marin Been was born on September the 5th of 1982 in Groningen, The Netherlands. In 2000 she completed her secondary school education at the Hendrik Pierson College, in Zetten. In September 2000 she started the Bachelor Psychology at the University of Maastricht. She obtained her masters degree in both Neuropsychology and Cognitive Neuroscience in 2005. In March 2006 she started a Ph.D project under the supervision of Prof. Dr. Peter De Weerd at the department of Cognitive Neuroscience at the Faculty of Psychology and Neuroscience of Maastricht University. 